\title{
On the iteration of
}

\section{quasimeromorphic mappings}

\author{
Luke Warren \\ Thesis submitted to The University of Nottingham \\ for the degree of Doctor of Philosophy
}

April 30, 2020

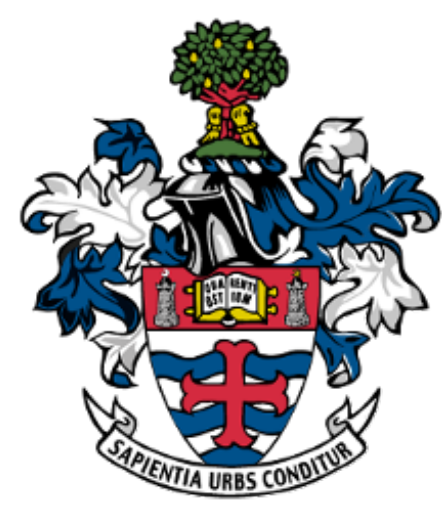


In memory of David Edward Williams (aka Gramps)

$07 / 10 / 1924-06 / 03 / 2017$ 


\section{Abstract}

This thesis is concerned with the iterative behaviour of quasimeromorphic mappings of transcendental type, which form higher-dimensional analogues of transcendental meromorphic functions on the complex plane. We extend classical Julia theory and results on escaping points from complex dynamics to the new setting. This complements recent dynamical advancements for quasiregular mappings, which are higher-dimensional analogues of holomorphic functions on the complex plane.

First, we define the Julia set for quasimeromorphic mappings of transcendental type and investigate its properties through two cases based on the cardinality of the backward orbit of infinity. To this end, we construct an example of a quasiregular mapping in dimension 3 with exactly one zero, subsequently showing that both cases arise. We then generalise an important growth result by Bergweiler to quasiregular mappings defined near an essential singularity. From this we show that many classical properties of the Julia set hold in our case; this includes proving a cardinality conjecture that remains open for general quasiregular mappings.

Next, we study the existence of escaping and non-escaping points in the new Julia set. In particular, following work by Nicks, we show that there exist points that escape arbitrarily slowly to infinity under iteration. Moreover we prove some basic relationships between the Julia set, the escaping set, the set of points whose orbit is bounded, and the set of points whose orbit is neither bounded nor tends to infinity. Finally, motivated by the work of Bolsch, we consider a class of mappings that is closed under composition and contains all quasimeromorphic mappings. Adapting 
previous methods, we show that the above results for quasimeromorphic mappings of transcendental type continue to hold for their iterates in a natural way. We also define a generalised escaping set, consisting of points whose orbits accumulate to some essential singularities or their pullbacks, and prove some existence results regarding points with specified accumulation sets. 


\section{Publications}

Much of the content of this thesis has previously appeared in the following publications:

[107] L. Warren: On the iteration of quasimeromorphic mappings; Math. Proc. Cambridge Philos. Soc.; 168(1), 1-11 (2020).

[108] L. Warren: On slow escaping and non-escaping points of quasimeromorphic mappings; Ergod. Th. \& Dynam. Sys.; [Online] Cambridge University Press; 1-27 (2020), available at doi:10.1017/etds.2019.110

[109] L. Warren: Constructing a quasiregular analogue of $z \exp (z)$ in dimension 3; submitted, 2019, available at https://arxiv.org/abs/1907.04720 


\section{Acknowledgements}

First and foremost, I would like to thank my supervisor Dr. Daniel Nicks for his support, dedication and boundless patience throughout my PhD journey. I am truly grateful for his continuous encouragement and guidance during each stage of my research and writing, and I feel incredibly fortunate to have been his first PhD student. I would also like to thank Dave Sixsmith for his thought-provoking discussions, ideas and feedback surrounding all things quasiregular. Alongside Dan, his valuable input really helped me find my footing in this area of mathematics.

I would like to thank all the researchers from the complex dynamics group that have been great sources of inspiration, advice and kindness in the academic world. I am grateful to Prof. Phil Rippon and Prof. Gwyneth Stallard for their advice, especially towards presenting talks and seminars. Some particular thanks goes to all the postgraduates and postdocs who have made conferences so much joy and helped me build confidence in the academic world, including Vasso, David, Fabrizio, Matthew, Simon, Leti, James, Tania, Jack, Argyris, Yiannis, Mohammed and Thanasis. Also extra thanks goes towards Iason, Myrto, Stavros and Dimitris (alongside Vasso), for your familial warmth and exuberance during my first international conferences, and hosting an unforgettable conference in Crete (CAFT, July 2018) where you

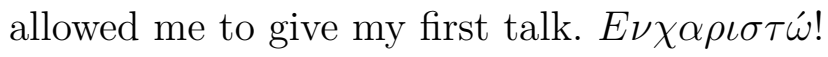

I would like to thank everyone at the School of Mathematics for making my time at the university so gratifying. Some particular thanks include, but is certainly 
not limited to: the 'lunch bunch' Jake, Juan, Fabio, Marco, George and Mauro, for some terribly funny puns and deep topical discussions; past and present members of the $\mathrm{C} 11$ office for their support and wisdom over the past three years (including fond memories of the pirate flag!); the Monday/Tuesday quizzers Cerian, David, Lawrence, Emma, Sam, Emily and Con, for some thoroughly enjoyable brain-stretching and amusement; and Charles for his generosity and valuable morale boosts leading up to submission.

Finally, I would like to give a huge thanks to my family and friends back home. Their constant encouragements through messages, calls and weekend visits were always revitalising and exactly what I needed to keep taking steps forward on the hardest days. This one is for you. 


\section{Contents}

1 Introduction and preliminaries $\quad 1$

1.1 Complex dynamics . . . . . . . . . . . . . . . . . . . 1

1.2 Quasiregular and quasimeromorphic mappings . . . . . . . . . 5

1.3 Fatou-Julia theory for quasiregular mappings . . . . . . . . . . . . . 16

1.4 Escaping sets . . . . . . . . . . . . . . . . 22

1.5 The Bolsch class $\mathbb{S} \ldots \ldots \ldots . \ldots 26$

1.6 Structure of thesis f . . . . . . . . . . . . . . . 30

2 Constructing a new quasiregular mapping in dimension $3 \quad 33$

2.1 Introduction . . . . . . . . . . . . . . . . 33

2.2 Value distribution for quasiregular and quasimeromorphic mappings 36

2.3 Proof of Theorem 2.1.2 . . . . . . . . . . . . . . . . . . . . . 38

2.3.1 Quasiregular mappings $Z$ and $g \ldots . . \ldots 39$

2.3.2 Construction of the quasiregular map $F \ldots . . . . . .40$

2.4 Modifying the construction of $F \ldots \ldots$. . . . . . . . . . 43

2.5 Dynamics of a family of quasiregular maps . . . . . . . . . . . 45

3 A growth result for quasiregular mappings near an essential 
3.1 Introduction . . . . . . . . . . . . . . . . . 51

3.1.1 Topological degree and a removability lemma . . . . . . . . 54

3.2 Proof of Theorem 3.1.2 . . . . . . . . . . . . . . . . . . 55

4 Julia sets of quasimeromorphic mappings of transcendental type 63

4.1 Introduction . . . . . . . . . . . . . . . . 63

4.1.1 Quasiregular mappings in $S$-punctured space . . . . . . . 67

4.2 Proof of Theorem 4.1 .3 when $\mathcal{O}_{f}^{-}(\infty)$ is finite . . . . . . . . . . . 69

4.3 Proof of Theorem 4.1.3 when $\mathcal{O}_{f}^{-}(\infty)$ is infinite . . . . . . . . . . . 71

4.4 Proof of Theorem 4.1.4 . . . . . . . . . . . . . . . . . . . . 74

5 On the existence of slow escaping and non-escaping points $\quad 79$

5.1 Introduction . . . . . . . . . . . . . . . . . . . . 79

5.1.1 An orbit lemma for quasimeromorphic mappings . . . . . . . 82

5.1.2 A holding-up lemma for quasimeromorphic mappings with finitely many poles . . . . . . . . . . . . . . . . 83

5.2 Proof of Theorem 5.1.1: Finitely many poles . . . . . . . . . . . . 84

5.2.1 Functions with the pits effect . . . . . . . . . . . 85

5.2.2 Functions without the pits effect. . . . . . . . . . . 89

5.2.3 A covering result for functions without the pits effect . . . . 97

5.3 Proof of Theorem 5.1.1: Infinitely many poles . . . . . . . . . . 100

5.4 Proof of Theorem 5.1.2(i) . . . . . . . . . . . . . . 105

5.4.1 Sufficient conditions for Theorem 5.1.2(i) . . . . . . . . 105 
5.4.2 Proof of Theorem 5.1.2(i) . . . . . . . . . . . . 106

5.5 Proof of Theorem 5.1.2(ii) . . . . . . . . . . . . . 108

5.5.1 Counterexamples . . . . . . . . . . . . . . 109

6 Quasimeromorphic mappings with countably many essential singularities

6.1 Introduction . . . . . . . . . . . . . . . . . . 113

6.2 Properties of mappings in $\mathbb{S}_{q m} \ldots \ldots . \ldots 116$

6.3 Julia set properties for $\mathbb{S}_{q m}$ mappings . . . . . . . . . . . . . . . . . 119

6.4 Escaping set of an essential singularity . . . . . . . . . . . . . 121

6.5 The generalised escaping set . . . . . . . . . . . . 126

6.5.1 Proof of Theorem 6.5.2 . . . . . . . . . . . . . . 128

6.5.2 Proof of Theorem 6.5.3 . . . . . . . . . . . . . . 138 


\section{$1 \quad$ Introduction and preliminaries}

The purpose of this chapter is to introduce the main definitions and ideas from the literature for use throughout the thesis. We first recall some basic definitions and results within complex dynamics for meromorphic functions on the complex plane, such as those for the Fatou set and the Julia set. We then introduce quasiregular and quasimeromorphic mappings, which form higher-dimensional analogues of entire functions and meromorphic functions respectively.

\subsection{Complex dynamics}

Complex dynamics is concerned with the study of the limiting behaviour under iteration of holomorphic functions and meromorphic functions on the complex plane $\mathbb{C}$ and the Riemann sphere $\hat{\mathbb{C}}=\mathbb{C} \cup\{\infty\}$. The foundation of the theory was first developed by Fatou $[32,33]$ and Julia [52] through studying the iteration of rational functions and entire functions.

The definitions of the Fatou set and Julia set have been extended and studied for many different types of functions, including transcendental entire functions, transcendental meromorphic functions, rational maps between Riemann surfaces and general classes of meromorphic functions that are closed under iteration; for 
example see [3, 28, 33], [5-8, 24, 90], [63] and [4, 21] respectively. Throughout this thesis, we shall always assume that the functions are non-constant and not linear transformations, unless otherwise stated. For notation, for each $n \in \mathbb{N}$ we write $f^{n}=f \circ f \circ \ldots \circ f$ to denote $f$ composed with itself $n$ times, and we set $f^{0}=$ id. Firstly, recall that a family of meromorphic mappings $\mathcal{M}$ defined on a domain $G \subset \hat{\mathbb{C}}$ is normal in $G$ if every sequence of mappings $\left(f_{n}\right)$ in $\mathcal{M}$ contains a subsequence that converges locally uniformly in $G$ in the spherical metric, either to a meromorphic function $g: G \rightarrow \hat{\mathbb{C}}$ or a constant $w \in \hat{\mathbb{C}}$. Further, $\mathcal{M}$ is normal at a point $z \in G$ if it is normal in some neighbourhood $U_{z} \subset G$ of $z$.

Definition 1.1.1. Let $f: \mathbb{C} \rightarrow \hat{\mathbb{C}}$ be a meromorphic function. Then the Fatou set $\mathcal{F}(f)$ is defined as

$$
\mathcal{F}(f)=\left\{z \in \hat{\mathbb{C}}:\left\{f^{n}: n \in \mathbb{N}\right\} \text { is well-defined and is normal at } z\right\},
$$

while the Julia set $\mathcal{J}(f)$ is defined as

$$
\mathcal{J}(f)=\hat{\mathbb{C}} \backslash \mathcal{F}(f)
$$

Informally, the Fatou set consists of points at which the iterates of the function exhibit stable behaviour, in the sense that small neighbourhoods of the points remain small with respect to the spherical metric under iteration. Conversely, the Julia set consists of points that are considered 'chaotic' in nature; the iterates at a point in the Julia set behave very differently to the iterates at points that are arbitrarily close.

For a holomorphic function, the definition of the Fatou set (and hence the Julia set) remains mostly unchanged; $\hat{\mathbb{C}}$ is replaced by $\mathbb{C}$, while the well-definedness condition for the family of iterates in the Fatou set is removed as there are no poles. Further, by the Arzela-Ascoli theorem there is an equivalent definition of 
the Fatou set, whereby a point $z \in \hat{\mathbb{C}}$ is in the Fatou set if the family of iterates are equicontinuous on some neighbourhood of $z$.

If a transcendental meromorphic function has at least two poles or has a single non-omitted pole, then it turns out we can remove the normality condition for the family of iterates and directly define the Fatou and Julia sets in terms of the preimages of infinity. To show this, we will require some notation and Montel's theorem.

Let $X, Y$ be sets, with $X \subset Y$, and suppose that $f: X \rightarrow Y$ is a function. Then for $y \in Y$, we define the backward orbit of $y$ as

$$
\mathcal{O}_{f}^{-}(y):=\bigcup_{m=0}^{\infty} f^{-m}(y)
$$

For $x \in X$, we define the forward orbit of $x$ as

$$
\mathcal{O}_{f}^{+}(x):=\bigcup_{m=0}^{\infty}\left\{f^{m}(x)\right\}
$$

where we set $f^{m}(x)=\varnothing$ when it is undefined. We further define the forward orbit of $U \subset X$ as

$$
\mathcal{O}_{f}^{+}(U):=\bigcup_{x \in U} \mathcal{O}_{f}^{+}(x)
$$

We let $\operatorname{card}(X)$ denote the cardinality of $X$. Further, we say that a set $X$ is forward invariant (under a mapping $f$ ) if $x \in X$ implies $f(x) \in X$, backward invariant if $f(x) \in X$ implies $x \in X$, and completely invariant if it is both forward and backward invariant.

Now suppose that $Y=\hat{\mathbb{C}}$ and $f: X \rightarrow \hat{\mathbb{C}}$ is a meromorphic function. Then for a point $x \in \mathcal{O}_{f}^{-}(\infty)$ we say that $x$ is an $N$-prepole if $f^{N}(x)=\infty$ for $N \in \mathbb{N}$. We also define the (Fatou) exceptional set $E(f)$ as the set of points whose backward orbit is finite. As a remark, if $f: \mathbb{C} \rightarrow \hat{\mathbb{C}}$ is a transcendental meromorphic function, then $\operatorname{card}(E(f)) \leq 2$ by Picard's theorem. For example the function $f(z)=\exp (z) / z$ 
has $E(f)=\{0, \infty\}$. Moreover if $x \in \mathbb{C}$ and $f: \mathbb{C} \rightarrow \hat{\mathbb{C}}$ is a transcendental meromorphic function, then $\mathcal{O}_{f}^{-}(x)$ is finite if and only if $\mathcal{O}_{f^{n}}^{-}(x)$ is finite for any $n \in \mathbb{N}$.

We now state Montel's theorem for meromorphic functions. In what follows, we use $d_{\chi}(z, w)$ to denote the spherical distance between $z, w \in \hat{\mathbb{C}}$.

Theorem 1.1.2 (Montel's Theorem). Let $\mathcal{F}$ be a family of meromorphic mappings on a domain $D \subset \hat{\mathbb{C}}$. Suppose that there exists some $\varepsilon>0$ such that each $f \in \mathcal{F}$ omits three distinct values $a_{1}(f), a_{2}(f), a_{3}(f) \in \hat{\mathbb{C}}$ with $d_{\chi}\left(a_{i}(f), a_{j}(f)\right) \geq \varepsilon$ for each $i \neq j$. Then $\mathcal{F}$ is a normal family on $D$.

Suppose that $f: \mathbb{C} \rightarrow \widehat{\mathbb{C}}$ is a transcendental meromorphic function and that $\operatorname{card}\left(\mathcal{O}_{f}^{-}(\infty)\right)>2$. It follows by Picard's theorem that $\mathcal{O}_{f}^{-}(\infty)$ is infinite. Then the largest open set where the family of iterates $\left\{f^{n}: n \in \mathbb{N}\right\}$ is defined is the set $\hat{\mathbb{C}} \backslash \overline{\mathcal{O}_{f}^{-}(\infty)}$. Since this set is forward invariant under $f$ and $\mathcal{O}_{f}^{-}(\infty)$ is infinite, then Montel's theorem implies that $\left\{f^{n}: n \in \mathbb{N}\right\}$ is normal there. Therefore

$$
\mathcal{F}(f)=\hat{\mathbb{C}} \backslash \overline{\mathcal{O}_{f}^{-}(\infty)} \text { and } \mathcal{J}(f)=\overline{\mathcal{O}_{f}^{-}(\infty)}
$$

Using tools such as Montel's theorem and Picard's theorem, it can be shown that the Fatou and Julia sets of meromorphic functions have many nice properties that are analogous to those of rational functions. We briefly summarise some key properties of the Julia set in the context of transcendental meromorphic functions below; see for example [9].

Theorem 1.1.3. Let $f: \mathbb{C} \rightarrow \hat{\mathbb{C}}$ be a transcendental meromorphic function. Then the following hold.

(i) $\mathcal{J}(f)$ is closed, infinite and does not contain any isolated points.

(ii) $x \in \mathcal{J}(f) \backslash\{\infty\}$ if and only if $f(x) \in \mathcal{J}(f)$. In particular, $\mathcal{J}(f) \backslash \mathcal{O}_{f}^{-}(\infty)$ is 
completely invariant.

(iii) $\mathcal{J}(f) \subset \overline{\mathcal{O}_{f}^{-}(x)}$ for every $x \in \hat{\mathbb{C}} \backslash E(f)$.

(iv) $\mathcal{J}(f)=\overline{\mathcal{O}_{f}^{-}(x)}$ for every $x \in \mathcal{J}(f) \backslash E(f)$.

(v) If $U \subset \hat{\mathbb{C}}$ is an open set such that $U \cap \mathcal{J}(f) \neq \varnothing$, then $\hat{\mathbb{C}} \backslash E(f) \subset \mathcal{O}_{f}^{+}(U)$.

(vi) For each $n \in \mathbb{N}$, if $\operatorname{card}\left(\mathcal{O}_{f}^{-}(\infty)\right)=1$, then $\mathcal{J}(f)=\mathcal{J}\left(f^{n}\right)$. Otherwise, if $\operatorname{card}\left(\mathcal{O}_{f}^{-}(\infty)\right)>2$, then $\mathcal{J}(f)=\overline{\mathcal{O}_{f}^{-}(\infty)}=\overline{\mathcal{O}_{f^{n}}^{-}(\infty)}$

(vii) $\mathcal{J}(f)$ is the closure of the set of repelling periodic points of $f$.

In the literature, (v) is sometimes referred to as the 'blowing-up' property. Furthermore, in (vi) we could also write $\mathcal{J}(f)=\mathcal{J}\left(f^{n}\right)$ when $\mathcal{O}_{f}^{-}(\infty)$ is infinite if we first define the Fatou set with respect to the Bolsch class $\mathbb{S}$ as in [21]; this is a class of functions that are meromorphic outside a countable set of essential singularities and closed under composition (see Section 1.5).

\subsection{Quasiregular and quasimeromorphic mappings}

As holomorphic functions and meromorphic functions are defined on $\mathbb{C}$, a natural question to ask is whether there is a generalisation of these functions in higher dimensions. One possible extension is to consider functions of several complex variables on $\mathbb{C}^{k}, k \geq 1$, that can be written (locally) as power series that converge on their domains. Although these functions enjoy a fruitful theory in the literature, when $k>1$ they do not have natural analogues of many classical results such as Picard's theorem. Moreover, a result of Liouville [54] shows that for $d \geq 3$ any sufficiently smooth conformal mapping from a domain in $\mathbb{R}^{d}$ to $\mathbb{R}^{d}$ is a restriction of a Möbius transformation; see [18] or [48] for a more modern monograph. 
In light of Liouville's theorem, it was asked how far the assumptions could be relaxed on the mappings whilst retaining the same rigidity result; this led to the study of Sobolev mappings.

Let $d \geq 2$, let $G \subset \mathbb{R}^{d}$ be a domain and let $f=\left(f_{1}, f_{2}, \ldots, f_{d}\right): G \rightarrow \mathbb{R}^{d}$. For notation, we will denote the $d$-dimensional Lebesgue measure in $\mathbb{R}^{d}$ by $m_{d}$.

Let $j \in\{1,2, \ldots, d\}$ be fixed and suppose that $f_{j} \in L_{l o c}^{1}(G)$. Then for $i=1,2, \ldots, d$, the function $g_{i, j} \in L_{l o c}^{1}(G)$ is the $i$ th first order weak partial derivative of $f_{j}$ if

$$
\int_{G} f_{j} D_{i} \phi d m_{d}=-\int_{G} g_{i, j} \phi d m_{d}
$$

for all test functions $\phi \in C^{\infty}(G)$ with compact support, where $D_{i}$ denotes the partial derivative with respect to the $i$ th coordinate direction.

Definition 1.2.1. Let $d \geq 2$ and $G \subset \mathbb{R}^{d}$ be a domain. Then the (local) Sobolev space $W_{d, l o c}^{1}(G)$ consists of all functions $f=\left(f_{1}, f_{2}, \ldots, f_{d}\right): G \rightarrow \mathbb{R}^{d}$ for which all first order weak partial derivatives exist and are locally $L^{d}$-integrable.

It was shown, for instance in [40, Lemma 1.11], that if $f: G \rightarrow \mathbb{R}^{d}$ is continuous and in $W_{p, l o c}^{1}(G)$, then the weak partial derivatives and the classical partial derivatives coincide almost everywhere. We refer to [40] for further information on Sobolev mappings.

A closely related concept to Sobolev spaces is that of absolute continuity on lines, which we shall define following [87]. First, let $Q=\left[a_{1}, b_{1}\right] \times\left[a_{2}, b_{2}\right] \times \cdots \times\left[a_{d}, b_{d}\right] \subset \mathbb{R}^{d}$ be a closed $d$-interval. Then a function $f: Q \rightarrow \mathbb{R}^{d}$ is absolutely continuous on lines $(\operatorname{ACL}(Q))$ if $f$ is absolutely continuous on almost every line segment in $Q$ parallel to the coordinates axes. If $G \subset \mathbb{R}^{d}$ is a domain, then a function $f: G \rightarrow \mathbb{R}^{d}$ is $\operatorname{ACL}(G)$ if $f$ is $\operatorname{ACL}(Q)$ for all closed $d$-intervals $Q \subset G$. It is known that for such mappings, the partial derivatives $D_{i} f(x)$ exist for all $i=1,2, \ldots, d$ and almost every $x \in G$. If $D_{i} f(x) \in L_{l o c}^{d}(G)$ as well, then we say that $f$ is $\operatorname{ACL}^{d}(G)$. 
Now it was shown for instance in [87, Proposition I.1.2] that $f: G \rightarrow \mathbb{R}^{d}$ is $\operatorname{ACL}^{d}(G)$ if and only if $f \in W_{d, l o c}^{1}(G)$ and is continuous, whereby the weak and ordinary partial derivatives coincide almost everywhere.

The study of continuous, injective Sobolev mappings with bounded distortion was first systematically performed by authors such as Gehring [36, 37] and Väisälä [101]; these maps are referred to as quasiconformal mappings in modern literature and form a generalisation of conformal mappings in higher dimensions. After this, Reshetnyak then developed the foundational theory of so-called quasiregular mappings in dimensions $d \geq 2$, through a series of papers, such as [78, 79, 81, 82]; these quasiregular mappings need not be injective.

From there, the theory surrounding the properties of quasiregular and so-called quasimeromorphic mappings was developed through a different approach by Martio, Rickman and Väisälä in [58-60], where several natural analogues of classical results for holomorphic functions and meromorphic functions were shown to hold. These quasiregular and quasimeromorphic mappings are hence considered to be the higher-dimensional analogues of holomorphic functions and meromorphic functions in $\mathbb{R}^{d}, d \geq 2$, respectively. Many other contributions to the quasiregular and quasimeromorphic theory have been made by authors such as Zorich, Iwaniec, Martin and others, whereby numerous analogous results have been attained to those found in complex analysis (cf. [19, 47-51, 61, 84, 104, 111]).

We shall now define quasiregular and quasimeromorphic mappings and give some basic results and examples for use within the thesis. Further details surrounding these mappings can be found in [87] and [105].

Definition 1.2.2. Let $d \geq 2$ and $G \subset \mathbb{R}^{d}$ be a domain. A continuous map $f: G \rightarrow \mathbb{R}^{d}$ in $W_{d, l o c}^{1}(G)$ is called quasiregular if there exists some constant $K_{1} \geq 1$ such that

$$
|D f(x)|^{d} \leq K_{1} J_{f}(x) \text { a.e. }
$$


where $D f(x)$ is the (formal) derivative of $f(x),|D f(x)|:=\left(\sup _{|v|=1}|D f(x)(v)|\right)$ denotes its norm and $J_{f}(x)$ denotes the Jacobian determinant.

If $f$ is quasiregular, then it also follows that there exists some $K_{2} \geq 1$ such that

$$
K_{2}\left(\inf _{|v|=1}|D f(x)(v)|\right)^{d} \geq J_{f}(x) \text { a.e. }
$$

The smallest constants $K_{1}$ and $K_{2}$ for which equations (1.2.1) and (1.2.2) hold are called the outer and inner dilatation of $f$, and are denoted $K_{O}(f)$ and $K_{I}(f)$ respectively. If $K=K(f) \geq 1$ is such that $\max \left\{K_{O}(f), K_{I}(f)\right\} \leq K$, then we say that $f$ is $K$-quasiregular. We note that in dimension two, we have $K_{O}(f)=K_{I}(f)$. Geometrically, quasiregular mappings send infinitesimally small spheres to infinitesimally small ellipsoids with bounded eccentricity, whilst preserving orientation. In this respect, it is easy to see that in dimension two, all holomorphic functions are 1-quasiregular. On top of this, the converse is also true (cf. [2]), completing the generalisation of the complex functions to the new setting. Further, it should be noted that $K$-quasiregular mappings are defined exactly the same way as $K$ quasiconformal mappings, without the injectivity requirement (cf. [105, p.128]).

From here, the concept of quasiregularity can be further extended. Consider the one point compactification $\hat{\mathbb{R}}^{d}=\mathbb{R}^{d} \cup\{\infty\}$ endowed with the spherical metric, obtained via the stereographic projection from the unit sphere in $\mathbb{R}^{d+1}$. Now let $M: \hat{\mathbb{R}}^{d} \rightarrow \hat{\mathbb{R}}^{d}$ be a sense-preserving Möbius transformation such that $M(\infty) \in \mathbb{R}^{d}$. We say that a continuous mapping $f$ is $K$-quasiregular at infinity if there exists a neighbourhood $U$ of $M(\infty)$ such that $f \circ M^{-1}$ is $K$-quasiregular on $U$. Further, for $x \in \hat{\mathbb{R}}^{d}$ we say that $f$ is $K$-quasimeromorphic at $x$ if either $f$ is $K$-quasiregular on some neighbourhood of $x$, or $f(x)=\infty$ and $M \circ f$ is $K$-quasiregular on some neighbourhood of $x$.

It was shown in [58] that these definitions are independent of the choice of $M$, thus 
are well-defined. Further, this definition is comparable to that of a meromorphic mapping on $\hat{\mathbb{C}}$. Finally, we note that we can equivalently define quasiregular mappings above by replacing the Sobolev space and continuity condition with $\mathrm{ACL}^{d}$, as equation (1.2.1) need only hold almost everywhere.

We will now consider some examples of quasiregular and quasimeromorphic mappings. The first example will be of a Zorich-type map, which is an adaptation of a quasiregular mapping introduced by Zorich in [111]; these form the quasiregular analogues of the exponential map $z \mapsto \exp (z)$. Here, we shall give an explicit example of a Zorich-type map in dimension 3 first constructed in [69, Section 5], for use within Chapter 2. It should be noted that the more general Zorich maps in $\mathbb{R}^{d}$, $d \geq 2$ are constructed by replacing $h(z)$ below with a bi-Lipschitz mapping from the $(d-1)$-cube $[0,1]^{d-1} \subset \mathbb{R}^{d-1}$ to the upper half sphere $\left\{y \in \mathbb{R}^{d}:|y|=1, y_{d} \geq 0\right\}$; see for instance [48, Section 6].

Example 1.2.3. Let $x=\left(x_{1}, x_{2}, x_{3}\right) \in[-1,1]^{2} \times \mathbb{R}$, let $h:[-1,1]^{2} \rightarrow \mathbb{R}^{3}$ be the map defined by $h\left(x_{1}, x_{2}\right)=\left(x_{1}, x_{2}, 1-\max \left\{\left|x_{1}\right|,\left|x_{2}\right|\right\}\right)$, and further define $Z:[-1,1]^{2} \times \mathbb{R} \rightarrow\left\{y \in \mathbb{R}^{3}: y_{3} \geq 0\right\}$ by

$$
Z(x):=\exp \left(x_{3}\right) h\left(x_{1}, x_{2}\right)
$$

Now on the domain $(-1,1)^{2} \times \mathbb{R}$,

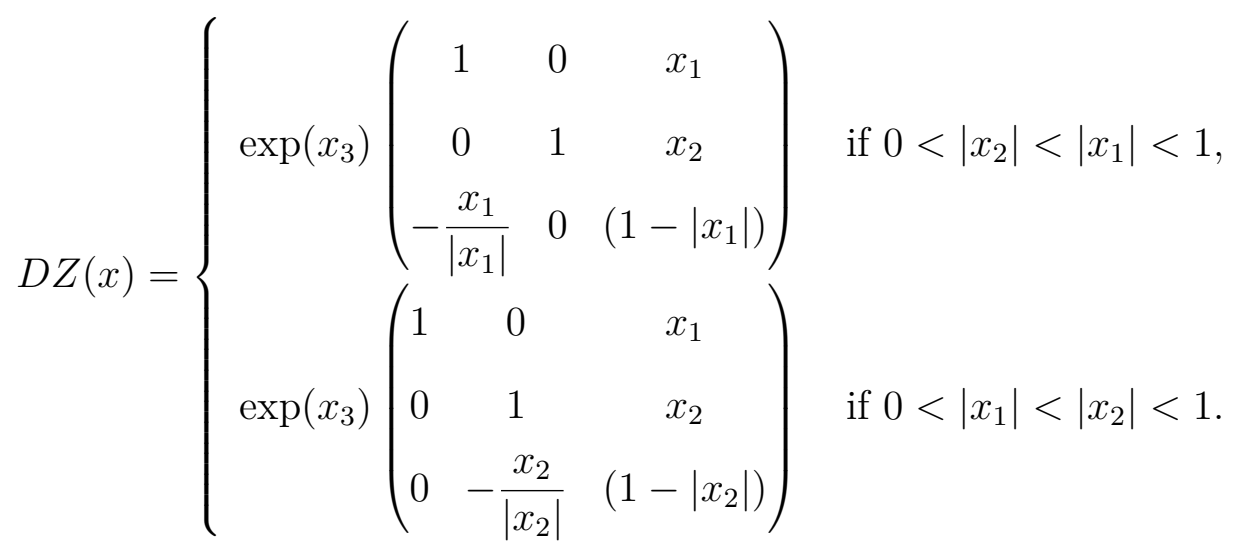


It follows that $|D Z(x)|^{3} \leq 24 \sqrt{3} \exp \left(3 x_{3}\right)$ and $J_{Z}(x)=\exp \left(3 x_{3}\right)$ for almost every $x \in(-1,1)^{2} \times \mathbb{R}$.

Next we extend $Z$ to be defined on $\mathbb{R}^{3}$ via reflections; for every reflection in the face of the domain, we reflect in the plane $\left\{\left(y_{1}, y_{2}, 0\right): y_{1}, y_{2} \in \mathbb{R}\right\}$ in the image. The resulting map is quasiregular as these reflections do not change the calculated values of $J_{Z}(x)$ and $|D Z(x)|$ in each reflected domain, and $Z$ is $\mathrm{ACL}^{3}$ in $\mathbb{R}^{3}$. Therefore $Z: \mathbb{R}^{3} \rightarrow \mathbb{R}^{3} \backslash\{0\}$ is a quasiregular mapping with an essential singularity at infinity (that is, $Z(x)$ does not have a limit as $x \rightarrow \infty$ ).

We now observe some useful properties of $Z$. Firstly if $w=\left(w_{1}, w_{2}, w_{3}\right) \in \mathbb{R}^{3}$, then reflecting in the plane $\left\{\left(-1, y_{2}, y_{3}\right): y_{2}, y_{3} \in \mathbb{R}\right\}$ will give us the point $\left(-w_{1}-2, w_{2}, w_{3}\right)$. Now a reflection in the plane $\left\{\left(1, y_{2}, y_{3}\right): y_{2}, y_{3} \in \mathbb{R}\right\}$ yields the point $\left(w_{1}+4, w_{2}, w_{3}\right)=w+(4,0,0)$. As we have reflected twice in the domain, then by the definition of $Z$ we would reflect twice in the image and return to the same point. This means that

$$
Z(w)=Z(w+(4,0,0))
$$

showing that $Z$ is periodic in the $x_{1}$ direction with period 4 . A similar argument can be used to show that $Z$ is periodic in the $x_{2}$ coordinate with period 4 .

Another useful property of $Z$ is that it satisfies the rotation relation

$$
Z\left(R_{(1,1)}(x)\right)=Z(x)
$$

where $R_{(1,1)}(x)$ denotes the point reached after rotating the point $x$ by $\pi$ radians around the line $\{(1,1, t): t \in \mathbb{R}\}$; see Section 2.3.1. Indeed, this is clear since such a rotation is the same as one reflection in the plane $\left\{\left(1, y_{2}, y_{3}\right): y_{2}, y_{3} \in \mathbb{R}\right\}$ followed by a reflection in the plane $\left\{\left(y_{1}, 1, y_{3}\right): y_{1}, y_{3} \in \mathbb{R}\right\}$. Once again, as we have reflected twice in the domain, then the images under $Z$ will be the same. 
Finally, it can be seen from the definition of $Z$ that if $x \in \mathbb{R}^{3}$ and $c \in \mathbb{R}$, then

$$
Z(x+(0,0, c))=\exp (c) Z(x)
$$

Next for an example of a quasimeromorphic mapping, Martio and Srebro in [62] constructed an example that is periodic in all coordinate directions. The construction is similar to that of the generalised version of the Zorich map above.

Example 1.2.4. Let $d \geq 2$ and let $T=[0,1]^{d}$ be a $d$-cube in $\mathbb{R}^{d}$. Let $x_{0} \in T$ denote the centre of the cube and let $f_{1}: B\left(x_{0}, 1 / 2\right) \rightarrow \operatorname{int}(T)$ be the radial stretching map starting from $x_{0}$, which is quasiconformal. Let $f_{2}: B\left(x_{0}, 1 / 2\right) \rightarrow\left\{y \in \mathbb{R}^{d}: y_{d}>0\right\}$ be a Möbius mapping.

Now consider the mapping $\wp=f_{2} \circ f_{1}^{-1}$ and extend $\wp$ to be defined on the whole of $\mathbb{R}^{d}$ by repeatedly reflecting in the plane $\left\{y \in \mathbb{R}^{d}: y_{d}=0\right\}$ and the faces of $T$ as done when defining the Zorich map. Then $\wp: \mathbb{R}^{d} \rightarrow \hat{\mathbb{R}}^{d}$ is a quasimeromorphic mapping with an essential singularity at infinity, with infinitely many poles, and is periodic in every coordinate direction.

In $[58$, Theorem 8.1] it was shown that the composition of two quasiregular mappings $f, g$, is itself a quasiregular mapping when $f$ is defined in the range of $g$, and the dilatations of $f \circ g$ satisfy

$$
K_{O}(f \circ g) \leq K_{O}(f) K_{O}(g), \text { and } K_{I}(f \circ g) \leq K_{I}(f) K_{I}(g)
$$

Similarly, if $g$ is a quasiregular mapping and $f$ is a quasimeromorphic mapping defined in the range of $g$, then $f \circ g$ is quasimeromorphic and the same inequality as above holds. As a consequence it is possible to consider the iteration of quasiregular mappings and quasimeromorphic mappings by restricting the function away from 
the poles before composition.

Although we can compose quasiregular and quasimeromorphic mappings together, we cannot expect the pointwise addition of two quasiregular mappings to be quasiregular. As a basic example, consider $(x, y) \mapsto(x, 2 y)$ and $(x, y) \mapsto(-x,-y)$, which are clearly quasiregular mappings in the plane. However, their pointwise addition creates the map $(x, y) \mapsto(0, y)$ which is clearly not quasiregular. In general, the issue arises when the derivatives of two mappings are in some sense 'comparable in size' on a part of the domain, whereby the pointwise addition can cause cancellations to occur as in the example. However, if one of the quasiregular mappings 'dominates' the other in terms of their partial derivatives, then it is possible for their pointwise addition to be quasiregular.

The following example from [14, Section 6] gives sufficient conditions for the pointwise addition of two quasiregular mappings defined on a domain in the plane to be quasiregular; these will be used in Chapter 5 . We note that in dimension two, the dilatation of a $K$-quasiregular mapping $f: G \rightarrow \mathbb{C}$ can be explicitly calculated as

$$
K(f)=\operatorname{ess} \sup \left\{\frac{\left|f_{z}(z)\right|+\left|f_{\bar{z}}(z)\right|}{\left|f_{z}(z)\right|-\left|f_{\bar{z}}(z)\right|}: z \in G\right\}
$$

where $f_{z}=\frac{1}{2}\left(f_{x}-i f_{y}\right)$ and $f_{\bar{z}}=\frac{1}{2}\left(f_{x}+i f_{y}\right)$ are the Wirtinger derivatives that exist a.e. on $G$ (c.f. [2]).

Example 1.2.5. Let $G \subset \mathbb{C}$ be a domain and let $p: G \rightarrow \mathbb{C}$ be a $K$-quasiregular mapping. Suppose that $\left|p_{z}\right|$ is bounded below wherever $p_{z}$ is defined on $G$, say by $M \in \mathbb{R}$. Now let $q: G \rightarrow \mathbb{C}$ be a continuous mapping in $W_{2, l o c}^{1}(G)$ whose partial derivatives $q_{x}$ and $q_{y}$ exist a.e. and are sufficiently small on $G$. We shall show that $p+q$ is quasiregular on $G$.

Indeed, first note that $p+q$ is continuous and in the local Sobolev space $W_{2, l o c}^{1}(G)$, 
so it suffices to show that the quantity

$$
K(p+q)=\operatorname{ess} \sup \left\{\frac{\left|(p+q)_{z}(z)\right|+\left|(p+q)_{\bar{z}}(z)\right|}{\left|(p+q)_{z}(z)\right|-\left|(p+q)_{\bar{z}}(z)\right|}: z \in G\right\}
$$

is bounded above by $K=K(p)$ as $\left|q_{x}\right|$ and $\left|q_{y}\right|$ tend to 0 , since $K(p) \geq 1$ is finite. First let $n \in \mathbb{N}$ be fixed and suppose that $\left|q_{x}\right|+\left|q_{y}\right| \leq M / n$ a.e. on $G$, so $\left|q_{\bar{z}}(z)\right|,\left|q_{z}(z)\right| \leq M / n \leq\left|p_{z}(z)\right| / n$ a.e. on $G$. Now by the linearity of the Wirtinger derivative, for a.e. $z \in G$ we have

$$
\begin{aligned}
& \left|(p+q)_{\bar{z}}(z)\right|=\left|p_{\bar{z}}(z)+q_{\bar{z}}(z)\right| \leq\left|p_{\bar{z}}(z)\right|+\frac{1}{n}\left|p_{z}(z)\right|, \\
& \left|(p+q)_{z}(z)\right| \leq\left|p_{z}(z)\right|+\left|q_{z}(z)\right| \leq\left(1+\frac{1}{n}\right)\left|p_{z}(z)\right|, \text { and } \\
& \left|(p+q)_{z}(z)\right| \geq|| p_{z}(z)|-| q_{z}(z)|| \geq\left(1-\frac{1}{n}\right)\left|p_{z}(z)\right| .
\end{aligned}
$$

It follows that,

$$
K(p+q) \leq \operatorname{ess} \sup \left\{\frac{(2 / n)\left|p_{z}(z)\right|+\left|p_{z}(z)\right|+\left|p_{\bar{z}}(z)\right|}{(-2 / n)\left|p_{z}(z)\right|+\left|p_{z}(z)\right|-\left|p_{\bar{z}}(z)\right|}: z \in G\right\}
$$

Finally, the right hand side of the above inequality tends to $K(p)$ as $n \rightarrow \infty$, so for sufficiently large $n$ we must have that $K(p+q)$ is finite. This is sufficient to conclude that $p+q$ is quasiregular on $G$ as required.

Let $G \subset \mathbb{R}^{d}$ be a domain and let $f: G \rightarrow \mathbb{R}^{d}$ be a quasiregular mapping, with $d \geq 2$. Then define the branch set

$$
B_{f}:=\{x \in G: f \text { is not locally homeomorphic at } x\} .
$$

If $d \geq 3, G=\mathbb{R}^{d}$ and $f$ is non-injective, then $B_{f} \neq \varnothing$; in fact, it is possible for $B_{f}$ to have positive topological dimension (in the sense of Menger and Urysohn; see [45, p.24]). However it was shown, for example in [102], that when $d \geq 3$ then $B_{f}$ 
and $f\left(B_{f}\right)$ have topological dimension at most $d-2$, and it was shown in [58] and [75] that $m_{d}\left(B_{f}\right)=m_{d}\left(f\left(B_{f}\right)\right)=0$.

As mentioned, quasiregular and quasimeromorphic mappings have many analogous properties to those in complex analysis. Here, recall that we consider only nonconstant maps. For instance, Reshetnyak showed in [78, 79] that quasiregular mappings are discrete, open, sense-preserving and differentiable almost everywhere. Another example comes in the form of a generalised version of Picard's theorem in the new setting, first established by Rickman in [83-85]. We shall state the theorem here with respect to quasimeromorphic mappings.

Denote the region between two spheres centred at the origin of radii $0 \leq r<s \leq \infty$, by

$$
A(r, s)=\left\{x \in \mathbb{R}^{d}: r<|x|<s\right\}
$$

Theorem 1.2.6. Let $d \geq 2$ and $K \geq 1$. Then there exists a constant $q=q(d, K)$, called Rickman's constant, such that the following hold:

(i) if $f: \mathbb{R}^{d} \rightarrow \hat{\mathbb{R}}^{d} \backslash\left\{a_{1}, a_{2}, \ldots, a_{q}\right\}$ is a $K$-quasimeromorphic mapping with the $a_{i} \in \hat{\mathbb{R}}^{d}$ distinct for $i=1,2, \ldots, q$, then $f$ is constant;

(ii) if $\rho>0$ and $f: A(\rho, \infty) \rightarrow \hat{\mathbb{R}}^{d} \backslash\left\{a_{1}, a_{2}, \ldots, a_{q}\right\}$ is a $K$-quasimeromorphic mapping with the $a_{i} \in \hat{\mathbb{R}}^{d}$ distinct for $i=1,2, \ldots, q$, then $f$ has a limit at $\infty$

In particular, suppose that $f$ is a K-quasimeromorphic mapping with an essential singularity at infinity and $X \subset \hat{\mathbb{R}}^{d}$ is a set with $\operatorname{card}(X) \geq q$. Then there exists some $x \in X$ such that $f^{-1}(x)$ is infinite.

Here we make several remarks. Firstly it is known that $q(2, K)=3$ for all dilatations $K \geq 1$, which agrees with the classical Picard theorem for meromorphic functions on $\mathbb{C}$. Next, for $K$-quasiregular mappings the quantity $q_{0}:=q-1$ is also referred to as Rickman's constant, to compensate for the fact that infinity is omitted. Where 
this constant is explicitly used, for instance in Chapter 5, we shall refer to it as Rickman's quasiregular constant. Further, by the above theorem the (Fatou) exceptional set $E(f)$ defined analogously for quasimeromorphic mappings has at most $q-1$ elements and $E(f)=E\left(f^{n}\right)$ for all $n \in \mathbb{N}$.

Let $f: \mathbb{R}^{d} \rightarrow \mathbb{R}^{d}$ be a $K$-quasiregular mapping. If $|f(x)| \rightarrow \infty$ as $x \rightarrow \infty$, then following Heinonen and Koskela [41], we say that $f$ is of polynomial type. Here, we can extend $f$ to be a quasiregular self-map of $\hat{\mathbb{R}}^{d}$ by setting $f(\infty):=\infty$. Otherwise, $f$ has an essential singularity at infinity and we say that $f$ is quasiregular of transcendental type. We also refer to quasimeromorphic mappings of transcendental type if they have an essential singularity at infinity. However, we note that it is possible for $f(x)$ to have a finite limit as $x$ tends to infinity, for instance with Möbius transformations.

For a quasimeromorphic mapping $f: \mathbb{R}^{d} \rightarrow \hat{\mathbb{R}}^{d}$, we define the degree of $f$ as

$$
\operatorname{deg}(f)=\max _{x \in \hat{\mathbb{R}}^{d}} \operatorname{card}\left(f^{-1}(x)\right)
$$

where we set $\operatorname{deg}(f)=\infty$ if $f^{-1}(x)$ is countably infinite for some $x \in \hat{\mathbb{R}}^{d}$. The degree of a quasiregular mapping is defined similarly, with $\hat{\mathbb{R}}^{d}$ replaced with $\mathbb{R}^{d}$. If $f$ is quasiregular of transcendental type, then $\operatorname{deg}(f)$ must be infinite by Theorem 1.2.6(ii). Furthermore the converse holds by the discreteness of $f$ and the fact that if the limit at infinity exists, then $f(\infty)=\infty$. Hence $f$ is quasiregular of transcendental type if and only if $\operatorname{deg}(f)$ is infinite. Equivalently, $f$ is of polynomial type if and only if $\operatorname{deg}(f)$ is finite.

We shall finally introduce two particularly useful families of sense-preserving Möbius maps for use throughout the thesis. First for each $y \in \hat{\mathbb{R}}^{d}$, we define $M_{y}: \hat{\mathbb{R}}^{d} \rightarrow \hat{\mathbb{R}}^{d}$ by

$$
M_{y}(x):= \begin{cases}x & \text { if } y=\infty \\ \phi\left(\frac{x-y}{|x-y|^{2}}\right) & \text { otherwise }\end{cases}
$$


where $\phi: \hat{\mathbb{R}}^{d} \rightarrow \hat{\mathbb{R}}^{d}$ is the reflection function

$$
\phi(x)=\phi\left(\left(x_{1}, x_{2}, \ldots, x_{d}\right)\right)=\left(-x_{1}, x_{2}, \ldots, x_{d}\right)
$$

It follows that $M_{y}(y)=\infty$ and

$$
M_{y}(\infty)= \begin{cases}\infty & \text { if } y=\infty \\ 0 & \text { otherwise }\end{cases}
$$

This Möbius map will be useful in Chapter 6 when we consider $y$ to be an essential singularity of some other function and want to consider the distance of points from $y$.

Next, for each $y \in \hat{\mathbb{R}}^{d}$ we define the Möbius map $\hat{M}_{y}: \hat{\mathbb{R}}^{d} \rightarrow \hat{\mathbb{R}}^{d}$ by

$$
\hat{M}_{y}(x):= \begin{cases}x & \text { if } y=\infty \\ M_{y}(x)+y & \text { otherwise }\end{cases}
$$

It should be noted that $\hat{M}_{y}(y)=\infty$ and $\hat{M}_{y}(\infty)=y$ for all $y \in \hat{\mathbb{R}}^{d}$.

\subsection{Fatou-Julia theory for quasiregular mappings}

As we can compose two quasiregular mappings or restricted quasimeromorphic mappings together, it is reasonable to consider whether the concepts and ideas from complex dynamics have a natural extension to the new setting. For example, are there analogous versions of the Fatou and Julia sets from Definition 1.1.1 in the new setting?

Recall that Montel's theorem, Theorem 1.1.2, is a key result used in complex dynamics to prove many of the classical properties of the Fatou set and the Julia 
set. In [64], Miniowitz was able to extend Montel's theorem to the quasiregular and quasimeromorphic setting. Here we shall state the result for quasiregular mappings and, for convenience, we shall also denote the spherical distance between two points $x_{1}, x_{2} \in \hat{\mathbb{R}}^{d}$ by $d_{\chi}\left(x_{1}, x_{2}\right)$.

Theorem 1.3.1. Let $\mathcal{F}$ be a family of $K$-quasiregular mappings on a domain $D \subset \mathbb{R}^{d}, d \geq 2$, and let $q_{0}=q_{0}(d, K)$ be Rickman's quasiregular constant.

Suppose that there exists some $\varepsilon>0$ such that each $f \in \mathcal{F}$ omits $q_{0}$ values $a_{1}(f), a_{2}(f), \ldots, a_{q_{0}}(f) \in \mathbb{R}^{d}$ with $d_{\chi}\left(a_{i}(f), \infty\right) \geq \varepsilon$ for all $i=1,2, \ldots, q_{0}$, and $d_{\chi}\left(a_{i}(f), a_{j}(f)\right) \geq \varepsilon$ for all $i \neq j$. Then $\mathcal{F}$ is a normal family on $D$.

For a $K$-quasiregular mapping $f: \mathbb{R}^{d} \rightarrow \mathbb{R}^{d}$, if $\left\{f^{n}: n \in \mathbb{N}\right\}$ forms a family of $K$-quasiregular mappings, then we can define the Fatou set and Julia set as in the classical setting. Using the above theorem, these will then have many analogous properties to their classical counterparts, for example those stated in Theorem 1.1.3(i)-(vi). $K$-quasiregular mappings whose iterates have the same dilatation $K$ are called uniformly quasiregular mappings, and surveys for these mappings can be found in [48] and [95]. As a remark, for $d \geq 3$ the only uniformly quasiregular mappings currently studied have been quasiregular self-maps of $\hat{\mathbb{R}}^{d}$, and it is unknown whether we can have a uniformly quasiregular mapping of transcendental type in this case; see [17].

From equation (1.2.3), for a general $K$-quasiregular mapping $f$ it is possible for the dilatation of the iterates $f^{k}$ to grow exponentially large without bound. This means that a sequence of iterates of a quasiregular map may converge locally uniformly to a limit function that is neither quasiregular nor a constant. Further to this, Theorem 1.3.1 cannot be applied to the family of iterates like Montel's theorem could. In this way, the Fatou and Julia sets defined using normality are not very useful to study in this setting.

We should remark that if $f$ is a general $K$-quasiregular mapping, then Theorem 1.3.1 
can be applied to a re-scaled family of mappings $\{f(r x) / s: r \in \mathbb{R}, s \in \mathbb{R} \backslash\{0\}\}$, since all members of this family will have the same dilatation $K$. We shall use this fact later in Chapter 2.

In attempting to establish an analogous iterative theory for non-uniformly quasiregular mappings, Sun and Yang [99, 100] (see also [11]) showed that some results of the Fatou-Julia theory still hold for a non-uniformly $K$-quasiregular map $f$ in dimension two, provided $K_{I}(f)<\operatorname{deg}(f)<\infty$. This was done by directly defining the Julia set using a version of the blowing-up property in Theorem 1.1.3(v), as

$$
\mathcal{J}(f):=\left\{z \in \hat{\mathbb{C}}: \operatorname{card}\left(\hat{\mathbb{C}} \backslash \mathcal{O}_{f}^{+}\left(U_{z}\right)\right) \leq 2 \text { for every neighbourhood } U_{z} \text { of } z\right\},
$$

and showing that $\mathcal{J}(f) \neq \varnothing$. In addition, Sun and Yang considered the complement $\mathcal{Q F}(f):=\hat{\mathbb{C}} \backslash \mathcal{J}(f)$, now called the quasi-Fatou set; by defining the Fatou set $\mathcal{F}(f)$ analogously to Definition 1.1.1, then $\mathcal{F}(f) \subset \mathcal{Q} \mathcal{F}(f)$.

It turned out that defining the Julia set this way, many analogous properties of the classical Julia set from complex dynamics could be recovered. For instance, the Julia set was shown to be completely invariant and $\mathcal{J}(f)=\mathcal{J}\left(f^{n}\right)$ for all $n \in \mathbb{N}$. Further the degree condition is strictly necessary as the winding map $f: \hat{\mathbb{C}} \rightarrow \hat{\mathbb{C}}$, given by $(r, \theta) \mapsto(r, 2 \theta)$ in polar coordinates, is such that $K_{I}(f)=\operatorname{deg}(f)=2$, while $\mathcal{J}(f)=\mathcal{F}(f)=\varnothing$ and $\mathcal{Q F}(f)=\hat{\mathbb{C}}$. It should be noted that Theorem 1.1.3(vii) is rather difficult to approach for non-uniformly quasiregular mappings, as there is currently no agreed analogue of what it means for a fixed point to be repelling for these maps. This contrasts the case for uniformly quasiregular mappings, where a definition was given by Hinkkanen, Martin and Mayer in [43] using the concept of a generalised derivative that agreed with other existing definitions. For further information, see [11].

Following the idea of Sun and Yang, Bergweiler [12] extended the definition of the 
Julia set (and hence the quasi-Fatou set) to non-uniformly quasiregular self-maps of $\hat{\mathbb{R}}^{d}$, with $d \geq 3$. This was done by replacing the cardinality condition in equation (1.3.1) with a condition using conformal capacity. Subsequently, Bergweiler and Nicks in [17] further extended the Julia set definition to include quasiregular mappings $f: \mathbb{R}^{d} \rightarrow \mathbb{R}^{d}$ of transcendental type as well.

Definition 1.3.2. For an open set $U \subset \mathbb{R}^{d}$ and a non-empty compact subset $C \subset U$, the pair $(U, C)$ is called a condenser. Its (conformal) capacity, denoted by $\operatorname{cap}(U, C)$, is then defined by

$$
\operatorname{cap}(U, C)=\inf _{u} \int_{U}|\nabla u|^{d} d m_{d}
$$

where the integral is with respect to the Lebesgue measure $m_{d}$, and the infimum is taken over all infinitely differentiable non-negative functions $u: \mathbb{R}^{d} \rightarrow \mathbb{R}$ such that $u(x)=1$ for all $x \in C$ and $u$ has compact support in $U$.

Reshetnyak [78] showed that if $\operatorname{cap}(A, C)=0$ for some bounded open set $A \supset C$, then $\operatorname{cap}\left(A^{\prime}, C\right)=0$ for every bounded open set $A^{\prime} \supset C$. In this case, we say that $C$ has zero capacity and write $\operatorname{cap}(C)=0$. Otherwise, we say that $C$ has positive capacity and write $\operatorname{cap}(C)>0$.

For an arbitrary closed subset $C \subset \hat{\mathbb{R}}^{d}$, we say that $C$ has zero capacity if for every non-empty compact subset $D \subset C \cap \mathbb{R}^{d}$, we have $\operatorname{cap}(D)=0$. It is known, say from [106], that finite sets have zero capacity, and sets with zero capacity have Hausdorff dimension zero. In this sense, sets of zero capacity are 'small'. Finally, the following was shown by Martio, Rickman and Väisälä in [58].

Lemma 1.3.3. Suppose that $D \subset \mathbb{R}^{d}$ is a domain, $f: D \rightarrow \mathbb{R}^{d}$ is a quasiregular map and $\operatorname{cap}(C)=0$ for some $C \subset D$. Then $\operatorname{cap}(f(C))=0$.

We shall now state the Julia set definition for a quasiregular mapping of transcendental type from [17], noting that the definition for quasiregular self-maps of $\hat{\mathbb{R}}^{d}$ 
is almost identical, with $\mathbb{R}^{d}$ replaced by $\hat{\mathbb{R}}^{d}$.

Definition 1.3.4. Let $d \geq 2$ and let $f: \mathbb{R}^{d} \rightarrow \mathbb{R}^{d}$ be a quasiregular mapping. Then the Julia set of $f$ is defined by

$$
\mathcal{J}(f)=\left\{x \in \mathbb{R}^{d}: \operatorname{cap}\left(\hat{\mathbb{R}}^{d} \backslash \mathcal{O}_{f}^{+}\left(U_{x}\right)\right)=0 \text { for every neighbourhood } U_{x} \text { of } x\right\} .
$$

Further, the quasi-Fatou set of a quasiregular mapping is defined by $\mathcal{Q F}(f)=$ $\mathbb{R}^{d} \backslash \mathcal{J}(f)$.

It has been shown in [12] that the Julia set definition coincides with the Julia set for uniformly $K$-quasiregular self-maps of $\hat{\mathbb{R}}^{d}$, and if $f$ is non-uniformly $K$-quasiregular with $\operatorname{deg}(f)>K_{I}(f)$, then $\mathcal{J}(f)$ is non-empty. Further to this, Bergweiler [12] and Bergweiler and Nicks [17] showed that for many non-uniformly quasiregular mappings, the Julia set has analogous properties to the classical Julia set for complex analytic functions. This will be summarised in the following theorem for quasiregular mappings of transcendental type.

Theorem 1.3.5. Let $d \geq 2$ and let $f: \mathbb{R}^{d} \rightarrow \mathbb{R}^{d}$ be a quasiregular mapping of transcendental type. Then the analogous conclusions of Theorem 1.1.3(ii)-(vi) hold if one of the following is satisfied:

(i) $d=2$,

(ii) $f$ is locally Lipschitz continuous,

(iii) $f$ does not have the pits effect (see Definition 5.2.1),

(iv) the local index of $f$ is bounded on $\mathbb{R}^{d}$ (see Section 2.1.1).

It has been conjectured in [17] that the above theorem holds for all quasiregular mappings of transcendental type, although this currently remains open. 
Another conjecture proposed in [17] is whether we can replace the capacity condition as in Definition 1.3.4 by a cardinality condition as used by Sun and Yang to define a Julia set. For a general quasiregular mapping, although we could immediately define a 'Julia set' using the cardinality condition, the difficulty arises when showing the set is non-empty.

By considering the set of exceptional points $E(f)$, an equivalent version of this conjecture can be formulated in the following way, here stated for quasiregular mappings.

Conjecture 1.3.6. Let $f: \mathbb{R}^{d} \rightarrow \mathbb{R}^{d}$ be a quasiregular mapping satisfying $\operatorname{deg}(f)>$ $K_{I}(f)$. Then $x \in E(f)$ if and only if $\operatorname{cap}\left(\overline{\mathcal{O}_{f}^{-}(x)}\right)=0$.

As a remark, the conjecture holds for quasiregular mappings of transcendental type that satisfy any of the conditions (i)-(iv) in Theorem 1.3.5.

More recently, Nicks and Sixsmith [70] have established and studied the Julia set for quasiregular mappings of punctured space, which form a higher dimensional analogue of holomorphic self-maps of $\mathbb{C}^{*}$; see Section 4.1.1 below. For these mappings it was shown that Conjecture 1.3.6 is true, providing some support for the conjecture holding for other non-uniformly quasiregular mappings. Further results and examples regarding the Fatou-Julia theory of non-uniformly quasiregular mappings can be found in $[12,34,35,67-69]$.

Throughout the remainder of this thesis, we shall not assume that the quasiregular and quasimeromorphic mappings are uniform.

In the direction of the Fatou-Julia theory for quasimeromorphic mappings, thus far in the literature only quasiregular self-maps of $\hat{\mathbb{R}}^{d}$ have been studied, such as by Bergweiler in [12]. For quasimeromorphic mappings of transcendental type, however, no such Fatou-Julia theory has existed and the Julia set was undefined. This was due to the current Julia set definition in Definition 1.3.4 being insufficient to deal with the behaviour of the poles, hence it would not coincide with the 
classical Julia set definition for transcendental meromorphic mappings.

The establishment and study of the Julia set for quasimeromorphic mappings of transcendental type will form the content of Chapter 4.

\subsection{Escaping sets}

Alongside the Fatou and Julia sets, Eremenko [28] introduced the escaping set for transcendental entire functions, which was later extended to transcendental meromorphic functions by Domínguez [24], and to holomorphic self-maps of the punctured plane $\mathbb{C}^{*}$ by Fang [31]. We shall provide the definition for transcendental meromorphic functions.

Definition 1.4.1. Let $f: \mathbb{C} \rightarrow \hat{\mathbb{C}}$ be a transcendental meromorphic function. Then the escaping set is defined as

$$
I(f)=\left\{z \in \mathbb{C}: f^{n}(z) \neq \infty \text { for all } n \in \mathbb{N}, f^{n}(z) \rightarrow \infty \text { as } n \rightarrow \infty\right\} .
$$

One reason for studying the escaping set is its strong relationship with the Julia set; this shall be stated for a transcendental meromorphic function and was shown in $[24]$.

Theorem 1.4.2. Let $f: \mathbb{C} \rightarrow \widehat{\mathbb{C}}$ be a transcendental meromorphic function. Then

(i) $I(f) \neq \varnothing$,

(ii) $I(f) \cap \mathcal{J}(f) \neq \varnothing$,

(iii) $\mathcal{J}(f)=\partial I(f)$, and

(iv) $I(f)$ is completely invariant. 
Due to this connection with the Julia set, the escaping set has been extensively studied with respect to its properties and global structure; see for example [30, 89, 92, 94, 96].

A particular interest with the escaping set is the rate of escape of points. This has prompted the introduction and study of numerous subsets of the escaping set. For example in [16], Bergweiler and Hinkkanen introduced the fast escaping set in the setting of entire functions. This set, informally, consists of points whose iterates escape to infinity 'as fast as possible' and has since been studied in detail in many different settings, for example see [56, 77, 91, 97]. Other sets of differing rates of escape have also been explored, for example in [88].

One question to ask is whether there exists a function where all the points in the escaping set are in the fast escaping set. This was answered by Rippon and Stallard in $[93]$ who showed that for any transcendental meromorphic function $f: \mathbb{C} \rightarrow \hat{\mathbb{C}}$, there always exists a point in $I(f) \cap \mathcal{J}(f)$ that escapes arbitrarily slowly.

Theorem 1.4.3. Let $f: \mathbb{C} \rightarrow \hat{\mathbb{C}}$ be a transcendental meromorphic function. Then for any positive sequence $a_{n} \rightarrow \infty$, there exists $\zeta \in \mathcal{J}(f)$ and $N \in \mathbb{N}$ such that $\left|f^{n}(\zeta)\right| \rightarrow \infty$ as $n \rightarrow \infty$, while also $\left|f^{n}(\zeta)\right| \leq a_{n}$ whenever $n \geq N$.

More recently the above result has been extended in the setting of transcendental entire functions, to get the slow escaping point to also lie in a tract; see [110].

Other related interests alongside the escaping set include the collections of points that do not escape to infinity, such as the bounded orbit set and the bungee set. For a transcendental meromorphic function $f: \mathbb{C} \rightarrow \hat{\mathbb{C}}$, these are defined as

$$
\begin{aligned}
& B O(f):=\left\{z \in \mathbb{C}:\left(f^{n}(z)\right)_{n \in \mathbb{N}} \text { is bounded }\right\}, \text { and } \\
& B U(f):=\mathbb{C} \backslash\left(I(f) \cup B O(f) \cup \mathcal{O}_{f}^{-}(\infty)\right) .
\end{aligned}
$$

$B O(f)$ consists of points whose forward orbit is bounded, whilst $B U(f)$ consists of 
points whose sequence of iterates contains both a bounded subsequence and a subsequence that tends to infinity. Together with $I(f)$ and $\mathcal{O}_{f}^{-}(\infty)$ when non-empty, these sets partition the space based on the behaviour of the forward orbit of the points. We note here that in the literature, the bounded orbit set for a transcendental meromorphic function $f$ is often denoted as $K(f)$ rather than $B O(f)$. In this thesis we shall not adopt this notation, as we reserve that notation for the dilatation of a quasiregular or quasimeromorphic mapping as in Definition 1.2.2. For a transcendental meromorphic function $f$, many properties of $B O(f)$ have been established; see for example $[28,73,93]$. Meanwhile, the set $B U(f)$ has been studied more recently for transcendental entire functions in $[74,98]$. It turns out that these sets share similar relationships with the Julia set as the escaping set. This is summarised below for transcendental entire functions.

Theorem 1.4.4. Let $f: \mathbb{C} \rightarrow \mathbb{C}$ be a transcendental entire function. Then

(i) $B O(f) \cap \mathcal{J}(f) \neq \varnothing$,

(ii) $B U(f) \cap \mathcal{J}(f) \neq \varnothing$, and

(iii) $\mathcal{J}(f)=\partial I(f)=\partial B O(f)=\partial B U(f)$.

Similar to the Fatou-Julia theory being extended to the quasiregular setting, the natural analogue of the escaping set has also been established and studied in the new setting. In [14], Bergweiler, Fletcher, Langley and Meyer extended Theorem 1.4.2(i) to quasiregular mappings of sufficient growth, and to quasimeromorphic mappings of transcendental type, while Bergweiler and Nicks [17] showed that Theorem 1.4.2(ii) held for quasiregular mappings of transcendental type. Further results have been found for quasiregular mappings of polynomial type by Fletcher and Nicks in [34].

The bounded orbit set $B O(f)$ and the bungee set $B U(f)$ have also been analogously defined and studied for quasiregular mappings of transcendental type in [17] 
and [71] respectively. Here, the analogue of Theorem 1.4.4(i) was established, with the analogue of Theorem 1.4.4(ii) shown to also hold for quasiregular mappings of transcendental type $f$ where $\operatorname{cap}(\mathcal{J}(f))>0$. However unlike the case with transcendental entire functions, examples of quasiregular mappings of transcendental type were constructed that showed that Theorem 1.4.4(iii), and subsequently Theorem 1.4.2(iii), need not hold; this is summarised below, where $\mathbb{C}$ is identified with $\mathbb{R}^{2}$ in the usual way.

Theorem 1.4.5. Let $f: \mathbb{R}^{d} \rightarrow \mathbb{R}^{d}$ be a quasiregular mapping of transcendental type. Then

(i) $I(f) \cap \mathcal{J}(f) \neq \varnothing, B O(f) \cap \mathcal{J}(f) \neq \varnothing$, and $\mathcal{J}(f) \subset \partial I(f) \cap \partial B O(f)$, and

(ii) if $\operatorname{cap}(\mathcal{J}(f))>0$, then $B U(f) \cap \mathcal{J}(f) \neq \varnothing$ and $\mathcal{J}(f) \subset \partial B U(f)$.

However there exist quasiregular mappings of transcendental type $f_{1}, f_{2}: \mathbb{C} \rightarrow \mathbb{C}$ such that

(iii) $\left(\partial I\left(f_{1}\right) \cap \partial B O\left(f_{1}\right)\right) \backslash J\left(f_{1}\right) \neq \varnothing$, and

(iv) $\partial B U\left(f_{2}\right) \backslash J\left(f_{2}\right) \neq \varnothing$.

The fast escaping set has been studied for quasiregular mappings of transcendental type in $[13,15]$. In this higher-dimensional setting, Nicks [66] was able to extend the slow-escape result by Rippon and Stallard to the case of quasiregular mappings of transcendental type $f: \mathbb{R}^{d} \rightarrow \mathbb{R}^{d}$.

Theorem 1.4.6. Let $d \geq 2$ and let $f: \mathbb{R}^{d} \rightarrow \mathbb{R}^{d}$ be a quasiregular mapping of transcendental type. Then for any positive sequence $a_{n} \rightarrow \infty$, there exists $\zeta \in \mathcal{J}(f)$ and $N \in \mathbb{N}$ such that $\left|f^{n}(\zeta)\right| \rightarrow \infty$ as $n \rightarrow \infty$, while also $\left|f^{n}(\zeta)\right| \leq a_{n}$ whenever $n \geq N$. 
For quasimeromorphic mappings of transcendental type $f$, thus far the only result known was that of [14], stating $I(f) \neq \varnothing$. Although the escaping set can be immediately defined and studied without requiring the Julia set, many results in the literature for escaping sets of quasiregular mappings and meromorphic functions involve Fatou-Julia theory.

In Chapter 5, we shall explore the escaping set, bounded orbit set and the bungee set for quasimeromorphic mappings of transcendental type in light of the new Julia set definition established in Chapter 4. In particular, there will be a focus on extending Theorem 1.4.5 and Theorem 1.4.6 to the new setting.

\subsection{The Bolsch class $\mathbb{S}$}

Suppose that $f: \mathbb{C} \rightarrow \hat{\mathbb{C}}$ is a transcendental meromorphic function. It follows that for $n \in \mathbb{N}$, then $f^{n}(z)$ is defined only when $z \in \hat{\mathbb{C}} \backslash \bigcup_{k=0}^{n-1} f^{-k}(\infty)$, hence the iterates $f^{n}$ may not in general be meromorphic on $\mathbb{C}$. Despite this, it has still been possible to get some Fatou-Julia results for $f^{n}$, such as Theorem 1.1.3(vi).

When studying the Fatou-Julia theory of transcendental meromorphic functions on the complex plane, it can therefore be useful to consider the smallest class of functions closed under composition that contains them. This class, denoted by $\mathbb{S}$, was established and studied by Bolsch in [20, 22], and is called the Bolsch class; see also [25]. Further generalisations of the Bolsch class have also been considered in $[4,42]$.

Definition 1.5.1. The Bolsch class $\mathbb{S}$ is defined as

$$
\begin{aligned}
& \mathbb{S}=\{f: \text { there exists a closed countable set } A(f) \subset \hat{\mathbb{C}} \text { such that } f \text { is } \\
&\text { meromorphic in } \hat{\mathbb{C}} \backslash A(f) \text { but in no larger set }\} .
\end{aligned}
$$


In the literature, the Bolsch class is also denoted by $\mathcal{K}$ (for example in [4]). As the notation $K$ is reserved for the dilatation of quasiregular and quasimeromorphic mappings, we shall use $\mathbb{S}$ as used by Bolsch in [21].

It was shown in [21] that $\mathbb{S}$ is indeed a class closed under composition and $A(f)$ consists of the set of isolated and non-isolated singularities of $f$, although Bolsch remarks that the argument method may have appeared first by Karlin and McGregor in [53] in a different context. We shall provide the proof here for completeness.

Theorem 1.5.2. Let $f, g \in \mathbb{S}$. Then the following hold.

(i) $A(f)=\overline{\{z \in \hat{\mathbb{C}}: z \text { is an isolated essential singularity of } f\}}$.

(ii) If $U_{z}$ is any open neighbourhood of a point $z \in A(f)$, then $f$ assumes in $U_{z} \backslash A(f)$ every value in $\hat{\mathbb{C}}$ infinitely often with at most two exceptions.

(iii) $f \circ g \in \mathbb{S}$, with $A(f \circ g)=A(g) \cup g^{-1}(A(f))$.

Proof. To prove (i), firstly note that any isolated point in $A(f)$ must be an essential singularity, as $f$ is not meromorphic in any larger set than $\hat{\mathbb{C}} \backslash A(f)$. Now it suffices to show that every non-isolated point in $A(f)$ is the limit of isolated points in $A(f)$. To this end, suppose for a contradiction that there exists a point $z_{0} \in A(f)$ and a ball $B\left(z_{0}, r\right)$ such that $B\left(z_{0}, r\right)$ does not contain any isolated points of $A(f)$. As $A(f)$ is countable, we may assume without loss of generality that $\partial B\left(z_{0}, r\right) \cap A(f)=\varnothing$, else we can make the ball smaller.

Now let $X=\overline{B\left(z_{0}, r\right)} \cap A(f)=B\left(z_{0}, r\right) \cap A(f)$, which is a non-empty complete metric space with respect to the natural subspace topology. As $A(f)$ is countable, then we can write $X$ as the countable union:

$$
X=\bigcup_{x \in B\left(z_{0}, r\right) \cap A(f)}\{x\} .
$$

As $X$ does not contain any isolated points of $A(f)$, then in the natural subspace 
topology, $\{x\}$ is nowhere dense in $X$ for all $x \in X$. However, this contradicts Baire's category theorem, as non-empty complete metric spaces cannot be written as the union of countably many nowhere-dense sets.

Next, (ii) follows from (i) and Picard's theorem.

Finally to prove (iii), first observe that $f \circ g$ is meromorphic outside of the set $A(g) \cup g^{-1}(A(f))$. It remains to show that $f \circ g$ is not meromorphic on any larger set. Indeed, let $z \in A(g)$ and let $U_{z}$ be a neighbourhood of $z$. By applying (ii) to $g$, then $g\left(U_{z} \backslash A(g)\right) \backslash A(f)$ is dense in $\hat{\mathbb{C}}$. Now applying (ii) to $f$, then $f\left(g\left(U_{z} \backslash A(g)\right) \backslash A(f)\right)$ is also dense in $\hat{\mathbb{C}}$. As $U_{z}$ was arbitrary, it follows that $f \circ g$ is not continuous at $z$. Next let $w \in g^{-1}(A(f)) \backslash A(g)$ and let $U_{w}$ be a neighborhood of $w$. Then $g\left(U_{w} \backslash A(g)\right)$ is a neighbourhood of $g(w) \in A(f)$. Applying (ii) to $f$, then $f\left(g\left(U_{w} \backslash A(g)\right) \backslash A(f)\right)$ is dense in $\hat{\mathbb{C}}$. As above, we can conclude that $f \circ g$ is not continuous at $w$, completing the proof of the theorem.

By extending Definition 1.1.1 in the obvious way to functions in $\mathbb{S}$, many analogous results of the Fatou-Julia theory for meromorphic functions have been extended to this class. For example, analogues of the properties listed in Theorem 1.1.3 hold, with infinity replaced by the essential singularity set where necessary; see $[4,20,21]$.

Alongside the Fatou and Julia sets being extended to the Bolsch class, it is possible to generalise the escaping set definition from Definition 1.4.1 to functions in class $\mathbb{S}$ as well. As infinity may not be the only essential singularity anymore, then we define escaping sets for each essential singularity; this will be done by following [4] and $[25]$.

Definition 1.5.3. Let $f \in \mathbb{S}$ and let $e \in A(f)$. Then the escaping set to $e$, denoted $I_{e}(f)$, is defined by

$$
I_{e}(f)=\left\{z \in \hat{\mathbb{C}}: f^{n}(z) \text { is defined for all } n \in \mathbb{N}, f^{n}(z) \rightarrow e \text { as } n \rightarrow \infty\right\}
$$


We remark that from the above definition, for a transcendental meromorphic function $f$ we have $I(f)=I_{\infty}(f)$.

Let $f \in \mathbb{S}$, let $e \in A(f)$ and let $z_{0} \in \hat{\mathbb{C}} \backslash \mathcal{O}_{f}^{-}(A(f))$. Then the omega limit set for $z_{0}$ under $f$, denoted by $\omega\left(z_{0}, f\right)$, is the set

$\omega\left(z_{0}, f\right)=\left\{z \in \hat{\mathbb{C}}: f^{n_{k}}\left(z_{0}\right) \rightarrow z\right.$ for some sequence of natural numbers $\left.n_{k} \rightarrow \infty\right\}$

Informally, the omega limit set for $z_{0}$ under $f$ is the collection of possible limit points of all subsequences of iterates of $z_{0}$ under $f$. As a remark, if $f$ is a transcendental entire or a transcendental meromorphic function, whereby $A(f)=\{\infty\}$, then $z_{0} \in B U(f)$ if and only if $\operatorname{card}\left(\omega\left(z_{0}, f\right)\right)>1$ and $\infty \in \omega\left(z_{0}, f\right)$.

Definition 1.5.4. Let $f \in \mathbb{S}$. Then the generalised escaping set, denoted $I_{g}(f)$, is defined as

$$
I_{g}(f)=\left\{z \in \hat{\mathbb{C}}: \omega(z, f) \subset \mathcal{O}_{f}^{-}(A(f))\right\} .
$$

It is immediately clear that if $f \in \mathbb{S}$ and $e \in A(f)$, then $I_{e}(f) \subset I_{g}(f)$. Further, it should be noted that if $f$ is a transcendental meromorphic function, then although it is possible that $B U(f) \cap I_{g}(f) \neq \varnothing$, it is not necessarily the case that $B U(f) \subset I_{g}(f)$. For example for the function $f(z)=\exp (z) / z$, we have $\mathcal{O}_{f}^{-}(A(f))=\{0, \infty\}$ and $f^{2}: \mathbb{C}^{*} \rightarrow \mathbb{C}^{*}$ is a transcendental self-map of the punctured plane. As we have $B U\left(f^{2}\right) \subset B U(f)$, then by [56, Theorem 1.1] it follows that there exists some $z \in B U(f)$ such that $\omega(z, f)=\{0, \infty\}$. However, it was also shown in [56, Section 4] that there exists a point $w \in B U(f)$ with $\omega(w, f) \supset\{a, \infty\}$ for some $a \in \mathbb{C}^{*}$. It was shown in [4] and [25] that for $f \in \mathbb{S}$, the analogues of Theorem 1.4.2 for $I_{e}(f)$ and $I_{g}(f)$ hold, respectively. In the particular case when $f$ is a holomorphic self-map of $\mathbb{C}^{*}$, for which $A(f)=\{0, \infty\}$, many results pertaining to different rates of escape have also been attained by Martí-Pete in [56], including the existence of fast escaping and slow escaping points. 
As quasimeromorphic mappings generalise meromorphic functions, then it makes sense to consider a higher-dimensional analogue of the Bolsch class $\mathbb{S}$ for quasimeromorphic mappings defined away from a countable set of essential singularities. Previously undefined within the literature, this quasimeromorphic Bolsch class, denoted by $\mathbb{S}_{q m}$, will be the object of study in Chapter 6 . By considering how the classical results for meromorphic mappings extended to functions in class $\mathbb{S}$, we shall aim to extend basic results for quasimeromorphic mappings to the new class $\mathbb{S}_{q m}$. This includes extending results regarding the escaping sets for functions in $\mathbb{S}_{q m}$, defined analogously to those in Definition 1.5.3 and Definition 1.5.4, such as showing the existence of slow escaping points and the existence of points with particular omega limit sets under the map.

\subsection{Structure of thesis}

While the iteration theory of quasiregular mappings is becoming more established in the literature, little is known about the iteration theory for quasimeromorphic mappings, in particular those of transcendental type. For instance, no Fatou-Julia theory for quasimeromorphic mappings of transcendental type has been established. The focus of this thesis is to explore the iteration theory of quasimeromorphic mappings of transcendental type in detail and extend recent results from quasiregular dynamics to the new setting.

In Chapter 2, we will construct the first explicit examples of quasimeromorphic mappings of transcendental type in $\mathbb{R}^{3}$ with a single omitted pole. This will be done by constructing the first example of a quasiregular mapping of transcendental type with exactly one zero. By suitably modifying the construction, we can create a family of such quasiregular mappings whose dynamics can be studied. In turn, this will allow us to study a family of quasimeromorphic mappings of transcendental type, for which we can identify explicit points that are in their bungee set. 
In Chapter 3 we shall develop a growth result for quasiregular mappings defined near an essential singularity, which generalises a result by Bergweiler in [10] for entire quasiregular maps of transcendental type. This new growth result will be used when showing the existence of slow escaping points in Chapter 5. Further, this growth result rectifies an omission in [70].

In Chapter 4 we shall define the Julia set for quasimeromorphic mappings of transcendental type with at least one pole, and show that this set satisfies many of the classical properties of the Julia set. This will be done by considering two distinct cases based on the cardinality of the backward orbit of infinity. When there is at least one pole and the backward orbit of infinity is finite, then a sufficiently large iterate of the function will be a quasiregular mapping of punctured space; these mappings were recently studied by Nicks and Sixsmith in [70]. By manipulating the Fatou-Julia theory for quasiregular mappings of punctured space, we are able to recover Fatou-Julia results for the original mapping.

When the backward orbit of infinity is infinite, the Julia set is defined as the closure of the backward orbit of infinity, which is parallel to the case for transcendental meromorphic functions. As Montel's theorem cannot be used in this setting, a more direct proof method is required to establish the properties of the Julia set. This chapter also includes a novel proof that for quasimeromorphic mappings of transcendental type with at least one pole, Conjecture 1.3.6 holds.

Within Chapter 5, we shall establish the existence of slow escaping points in the Julia set for quasimeromorphic mappings of transcendental type with at least one pole. This proof is split into two cases based on the number of poles, with the methods of Nicks in [66] being adapted to the case when there are finitely many. In the remaining case, a 'pole-hopping' technique used in [14] is adapted.

In addition, within Chapter 5 we shall explore the bounded orbit set and the bungee set in the new setting. This will include showing that these sets intersect the Julia 
set infinitely often and that the Julia set is contained within the boundary of these sets. However, by modifying existing examples in [17] and [71], we shall be able to construct examples of quasimeromorphic mappings of transcendental type that show the inclusion can be strict, analogous to the case with quasiregular mappings of transcendental type.

Finally, in Chapter 6, we shall define and explore the quasimeromorphic Bolsch class $\mathbb{S}_{q m}$, which is a generalisation of the Bolsch class $\mathbb{S}$ in the new setting. Some basic properties of this class will be verified, and then some results from Chapter 4 will be shown to generalise naturally to functions in $\mathbb{S}_{q m}$.

For functions within the quasimeromorphic Bolsch class $\mathbb{S}_{q m}$, generalised versions of the escaping set will also be defined and explored. Here, the existence of points escaping arbitrarily slowly to any essential singularity will be shown, and three open questions posed in [25] regarding the existence of points with particular omega limit sets will be answered. 


\section{Constructing a new quasiregular mapping in dimension 3}

The focus of this chapter is on the construction of a new quasiregular analogue of $z \mapsto z \exp (z)$ in dimension 3, which is the first explicit example of a quasiregular mapping of transcendental type with a non-omitted exceptional point. This, in turn, gives rise to the first example of a quasimeromorphic mapping of transcendental type where the backward orbit of infinity is non-empty and finite. In Chapter 4 we will develop a general iterative theory for quasimeromorphic mappings of transcendental type with at least one pole. There, we will often work in cases that depend on whether the backward orbit of infinity is finite or infinite. Examples constructed in this chapter show that the former case is not empty.

Much of the content forming this chapter can be found in [109].

\subsection{Introduction}

Recall from Section 1.1 that when studying the dynamical behaviour of transcendental meromorphic functions $f: \mathbb{C} \rightarrow \widehat{\mathbb{C}}$, we often consider different cases based on the backward orbit of infinity. For example, different techniques are often used 
when the backward orbit of infinity is infinite, such as with $z \mapsto \tan (z)$, compared to the case when it is finite, such as with $z \mapsto \exp (z) / z$.

There are many explicit examples of functions in complex dynamics that exhibit different dynamics. Rather surprisingly, in the literature there are comparatively few examples of quasiregular and quasimeromorphic mappings of transcendental type in general. This is despite value distribution results for quasiregular and quasimeromorphic mappings, such as those analogous to Nevanlinna and Ahlfors, and a function-theoretic result by Drasin and Pankka [27] showing that quasiregular mappings in $\mathbb{R}^{d}$ omitting a specified set of points can be constructed; see Section 2.2 below.

Explicit examples of quasimeromorphic mappings of transcendental type where the backward orbit of infinity is infinite have been constructed. For instance, Martio and Srebro's §-functions from Example 1.2.4 have infinitely many poles.

In dimension two, we can compose a suitable quasiconformal mapping and a transcendental meromorphic mapping together to get quasimeromorphic mappings of transcendental type where the backward orbit of infinity is finite.

Example 2.1.1. Consider the quasiconformal mapping $f: \mathbb{C} \rightarrow \mathbb{C}$ given by $f(z)=z|z|$, which has dilatation $K(f)=2$ and $f^{-1}(0)=\{0\}$. Let $g: \mathbb{C} \rightarrow \hat{\mathbb{C}}$ be the transcendental meromorphic function defined by $g(z)=\exp (z) / z$. Then the composition $\phi=g \circ f$ given by

$$
\phi(z)=\frac{\exp (z|z|)}{z|z|}
$$

is a quasimeromorphic mapping of transcendental type with dilatation $K(\phi)=2$ and $\mathcal{O}_{\phi}^{-}(\infty)=\{0, \infty\}$.

A natural question is whether examples of such quasimeromorphic mappings of transcendental type also exist in dimensions $d \geq 3$. A simple example would 
be a quasimeromorphic analogue of $z \mapsto \exp (z) / z$, although one has yet to be constructed. A major difficulty lies in the fact that in $\mathbb{R}^{d}$, with $d \geq 3$, there is no reasonable way to define commutative vector multiplication (as in $\mathbb{C}$ ).

A different method is to consider a quasiregular mapping of transcendental type $f: \mathbb{R}^{d} \rightarrow \mathbb{R}^{d}$ which takes a value $a \in E(f)$ at least once, but finitely often. Then we can compose $f$ with the sense-preserving Möbius map $\hat{M}_{a}$ defined as in equation (1.2.6). Consequently, our question reduces to the existence of such a quasiregular mapping. One of the simplest examples of such a quasiregular mapping would be a higher-dimensional analogue of $z \exp (z)$. However, no such quasiregular mappings of transcendental type have been constructed, even in dimension $d=3$, due to the same difficulty as before.

We shall construct the first explicit example of a quasiregular mapping of transcendental type $F: \mathbb{R}^{3} \rightarrow \mathbb{R}^{3}$ where an exceptional point is taken at least once, but finitely often. This will be done by constructing a quasiregular analogue of $z \exp (z)$ following the observation that we can write the complex function $z \exp (z)=\exp (G(\log (z)))$, where $G(z)=z+\exp (z)$. Then we will define our map as the composition $\mathcal{Z} \circ g \circ \mathcal{Z}^{-1}$, where $\mathcal{Z}$ is analogous to exp and $g$ is analogous to $G$.

Theorem 2.1.2. There exists a quasiregular mapping of transcendental type $F: \mathbb{R}^{3} \rightarrow \mathbb{R}^{3}$ such that

$$
F(x)=0 \text { if and only if } x=0 .
$$

As an immediate corollary we can provide the first example of a quasimeromorphic mapping of transcendental type in $\mathbb{R}^{3}$ with a single omitted pole. This consequently shows that such maps exist, justifying the necessity for the different techniques used in Chapter 4 when establishing and analysing the Julia set and its properties 
for quasimeromorphic mappings of transcendental type with at least one pole.

Corollary 2.1.3. There exists a quasimeromorphic map of transcendental type $f: \mathbb{R}^{3} \rightarrow \hat{\mathbb{R}}^{3}$ such that

$$
\mathcal{O}_{f}^{-}(\infty)=\{0, \infty\}
$$

The construction method used in the proof of Theorem 2.1 .2 readily generalises to create other quasiregular mappings that satisfy equation (2.1.1). In particular, we can exhibit a family of quasiregular mappings in dimension $d=3$ for which the quasi-Fatou set is connected and coincides with the attracting basin of 0. Using this quasiregular family, we can construct a particular family of quasimeromorphic mappings that satisfy equation (2.1.2), for which we can give explicit points whose iterates are neither bounded nor tend to infinity; this is summarised in the following result.

Theorem 2.1.4. There exists a family $\mathcal{F}$ of quasiregular mappings of transcendental type such that for all $f \in \mathcal{F}, f$ satisfies equation (2.1.1), $\mathcal{J}(f)$ contains half-rays and $\mathcal{Q F}(f)$ is connected.

Moreover, there exists a family $\mathcal{G}$ of quasimeromorphic mappings of transcendental type such that for all $g \in \mathcal{G}, g$ satisfies equation (2.1.2) and there exist half-rays on which the iterates of $g$ neither stay bounded nor tend to infinity.

\subsection{Value distribution for quasiregular and quasimeromorphic mappings}

To highlight the significance of the constructed functions in Theorem 2.1.2 and Corollary 2.1.3, we shall briefly discuss some results from function theory regarding the general value distribution of quasiregular and quasimeromorphic mappings.

For transcendental meromorphic mappings $f: \mathbb{C} \rightarrow \hat{\mathbb{C}}$, Nevanlinna theory can 
be used to study the distribution of values; this includes a defect relation which yields Picard's theorem as a corollary (see [39] and [65]). Ahlfors [1] developed a parallel theory to that of Nevanlinna which is more geometric in nature, including a pointwise version of the same defect relation. This version states that if $f$ is a transcendental meromorphic mapping, then there exists some small set $E \subset[1, \infty)$ such that for any distinct points $a_{1}, a_{2}, \ldots, a_{n} \in \hat{\mathbb{C}}$, then

$$
\limsup _{\substack{r \notin E, r \rightarrow \infty}} \sum_{i=1}^{n} \delta\left(a_{i}, r\right) \leq 2
$$

Here if $a \in \hat{\mathbb{C}}$ and $r>0$, then $\delta(a, r) \in[0,1]$, called the defect function of $a$, measures the rarity of the preimages of $a$ under $f$ against the average point of $\hat{\mathbb{C}}$ in the closed ball $\bar{B}(0, r)$. In particular if $a$ is an exceptional point of $f$, so $f^{-1}(a)$ is finite or empty, then $\delta(a, r) \rightarrow 1$ as $r \rightarrow \infty$. For example, for $z \mapsto \exp (z) / z$, then $\delta(0, r) \rightarrow 1$ and $\delta(\infty, r) \rightarrow 1$ as $r \rightarrow \infty$. Nonetheless, it is possible to have a point taken infinitely often but whose defect function still tends to 1 .

Example 2.2.1. Let $f: \mathbb{C} \rightarrow \mathbb{C}$ be defined by $f(z)=(\exp (z)-1) \exp (\exp (z))$. Then $f(z)=0$ if and only if $z=2 k \pi i$ for $k \in \mathbb{Z}$.

However, every point in $\mathbb{C} \backslash\{0\}$ has significantly more preimages than 0 in $B(0, r)$ for large values of $r>0$, due to the fast growth of $\exp (\exp (z))$. This means that the zeros of $f$ get rarer in comparison to the average point of $\mathbb{C}$ as $r$ increases, hence $\delta(0, r) \rightarrow 1$ as $r \rightarrow \infty$.

A converse result to equation (2.2.1) was proven by Drasin in [26]: for each $m \in \mathbb{N}$, let $a_{m} \in \hat{\mathbb{C}}$ be distinct points and let $\delta_{m} \in[0,1]$ with $\sum \delta_{m} \leq 2$. Then there exists some transcendental meromorphic function $g$ and some small set $E \subset[1, \infty)$ such that

$$
\lim _{r \rightarrow \infty, r \notin E} \delta(a, r)= \begin{cases}\delta_{m} & \text { if } a=a_{m} \text { for some } m \in \mathbb{N} \\ 0 & \text { otherwise }\end{cases}
$$


For a quasimeromorphic mapping of transcendental type $f: \mathbb{R}^{d} \rightarrow \hat{\mathbb{R}}^{d}$, Rickman [86] managed to generalise Ahlfors' result to establish a pointwise defect relation. Here equation (2.2.1) holds almost exactly, albeit with the value 2 replaced by Rickman's constant $q$ from Theorem 1.2.6. Furthermore in dimension $d=3$, Rickman extended Drasin's theorem to the quasimeromorphic setting, once again replacing the value 2 with the Rickman's constant $q$ as before. For further details, we refer to [85].

Analogous to the meromorphic setting, a corollary of Rickman's defect relation is Theorem 1.2.6(i). This result was shown to be sharp for dimension $d=3$ in [85] and, more recently, in all dimensions $d \geq 3$ by Drasin and Pankka [27]. They showed that given any $y_{1}, y_{2}, \ldots, y_{p} \in \mathbb{R}^{d}$, there exists a quasiregular map $f: \mathbb{R}^{d} \rightarrow \mathbb{R}^{d}$ omitting exactly $y_{1}, y_{2}, \ldots, y_{p}$. As a very simple example, Zorich-type maps such as that from Example 1.2.3 omit both 0 and $\infty$.

Although Drasin and Pankka's result can give us a quasiregular function with prescribed omitted points, unfortunately none of the results above can be directly applied to get a quasiregular or quasimeromorphic mapping in dimension $d \geq 3$ with at least one non-omitted exceptional point.

\subsection{Proof of Theorem 2.1.2}

To construct a quasiregular mapping of transcendental type $F: \mathbb{R}^{3} \rightarrow \mathbb{R}^{3}$ such that $F^{-1}(0)=\{0\}$, we shall first consider the composition of a quasiregular mapping of transcendental type $g: \mathbb{R}^{3} \rightarrow \mathbb{R}^{3}$ and the modified version of the Zorich mapping $Z: \mathbb{R}^{3} \rightarrow \mathbb{R}^{3} \backslash\{0\}$ from Example 1.2.3, both constructed by Nicks and Sixsmith in [69]. It will then follow that $f:=\hat{M}_{0} \circ F: \mathbb{R}^{3} \rightarrow \hat{\mathbb{R}}^{3}$ is a quasimeromorphic mapping of transcendental type satisfying equation (2.1.2), where $\hat{M}_{0}$ is the sense-preserving Möbius map defined via equation (1.2.6) with $d=3$. 


\subsubsection{Quasiregular mappings $Z$ and $g$}

Here, we shall introduce the mappings $Z$ and $g$ as mentioned above, along with some properties that will be useful within the construction of $F$. First we introduce some new notation.

For $u, v \in \mathbb{R}$, let $R_{(u, v)}(x)$ denote the point attained by rotating the point $x \in \mathbb{R}^{3}$ by $\pi$ radians around the line $\{(u, v, t): t \in \mathbb{R}\}$. Using this notation, observe that for any $x \in \mathbb{R}^{3}$ and any $\alpha, a, b \in \mathbb{R}$, if $y=R_{(u, v)}(x)$ for some $u, v \in \mathbb{R}$ then

$$
\alpha y+(a, b, 0)=\alpha R_{(u, v)}(x)+(a, b, 0)=R_{(\alpha u+a, \alpha v+b)}(\alpha x+(a, b, 0)) .
$$

Now, recall the Zorich-type mapping $Z: \mathbb{R}^{3} \rightarrow \mathbb{R}^{3} \backslash\{0\}$ constructed in Example 1.2.3, where

$$
Z(x):=\exp \left(x_{3}\right)\left(x_{1}, x_{2}, 1-\max \left\{\left|x_{1}\right|,\left|x_{2}\right|\right\}\right)
$$

for $x=\left(x_{1}, x_{2}, x_{3}\right) \in[-1,1]^{2} \times \mathbb{R}$, and the mapping was extended to be quasiregular on the whole of $\mathbb{R}^{3}$ by reflecting in the domain and image appropriately.

Further, recall that $Z$ was shown to be periodic in the $x_{1}$ and $x_{2}$ directions with period 4 and satisfy the relations

$$
\begin{gathered}
Z\left(R_{(1,1)}(x)\right)=Z(x), \text { and } \\
Z(x+(0,0, c))=\exp (c) Z(x) .
\end{gathered}
$$

for all $c \in \mathbb{R}$.

Now let $g: \mathbb{R}^{3} \rightarrow \mathbb{R}^{3}$ and $L>1$ respectively denote the quasiregular map of transcendental type and the constant from [69, Section 6]. It was shown there that using $Z$ as defined above, then $g$ has the following properties. To simplify notation, 
for $R \in \mathbb{R}$ we shall denote the upper half-space $\left\{\left(y_{1}, y_{2}, y_{3}\right) \in \mathbb{R}^{3}: y_{3}>R\right\}$ by $\mathbb{H}_{>R}$, and we further define the upper and lower half-spaces $\mathbb{H}_{\geq R}, \mathbb{H}_{\leq R}$ and $\mathbb{H}_{<R}$ similarly.

(G1) $g(x)=x$ when $x \in \mathbb{H}_{\leq 0}$,

(G2) $g(x)=x+Z(x)$ when $x \in \mathbb{H}_{\geq L}$,

(G3) $g(x+c)=g(x)+c$ for $c \in\{(4,0,0),(0,4,0)\}$, and

(G4) $g\left(R_{(2,2)}(x)\right)=R_{(2,2)}(g(x))$.

\subsubsection{Construction of the quasiregular map $F$}

By using the properties of $g$ and $Z$ above, we shall now proceed to the construction of $F$. First, define the translation $T: \mathbb{R}^{3} \rightarrow \mathbb{R}^{3}$ by $T(x)=x-(1,1,0)$. Now define $F: \mathbb{R}^{3} \rightarrow \mathbb{R}^{3}$ by setting $F(0)=0$, and for every $x \in \mathbb{R}^{3} \backslash\{0\}$ set

$$
F(x)=\left(Z \circ T \circ g \circ T^{-1} \circ \phi\right)(x),
$$

where $\phi$ is an inverse branch of $Z$. We claim that $F$ is independent of the choice of $\phi$ and that $F$ is a quasiregular mapping of transcendental type satisfying equation (2.1.1). Initially, we will assume that $F$ is independent of the choice of $\phi$. Note that $F^{-1}(0)=\{0\}$ and

$$
(F \circ(Z \circ T))(x)=((Z \circ T) \circ g)(x) \text { for all } x \in \mathbb{R}^{3} \backslash\{0\} \text {. }
$$

By property (G1) of $g$, we find that $\left(T^{-1} \circ \phi\right)(w)$ is a fixed point of $g$ for any given $w \in B(0,1 / 2) \backslash\{0\}$. It follows by construction that $F$ fixes all points in $B(0,1 / 2) \backslash\{0\}$, hence $F$ is continuous at 0 . Further, for each $x \in \mathbb{R}^{3} \backslash\{0\}$ we 
can choose an inverse branch $\phi$ such that $\phi$ is continuous at $x$. Now as $F$ is the composition of continuous mappings, we have that $F$ is also continuous on $\mathbb{R}^{3} \backslash\{0\}$. Next consider the branch set of $Z$. This will be given by the edges of the initial domain $[-1,1]^{2} \times \mathbb{R}$ and their reflections, so

$$
B_{Z}=\left\{\left(2 n+1,2 m+1, x_{3}\right): n, m \in \mathbb{Z}, x_{3} \in \mathbb{R}\right\}
$$

By a direct calculation, it follows that

$$
Z\left(B_{Z}\right) \cup\{0\}=\{t(1,1,0): t \in \mathbb{R}\} \cup\{t(-1,1,0): t \in \mathbb{R}\} .
$$

Let $V=Z\left(B_{Z}\right) \cup\{0\}$. Then there exists some $K \geq 1$ depending only on $Z$ such that for any $x \in \mathbb{R}^{3} \backslash V$, it is possible to choose an inverse branch $\phi$ of $Z$ that is locally $K$-quasiconformal on some neighbourhood $U_{x} \subset \mathbb{R}^{d} \backslash V$ of $x$. As the functions $Z$, $g, T$ and $T^{-1}$ are quasiregular and the inverse branch $\phi$ can be chosen to be locally $K$-quasiconformal, then $F$ will be quasiregular on $\mathbb{R}^{3} \backslash V$. Since $m_{2}(V)=0$ and $F$ is continuous on $\mathbb{R}^{3}$, it follows that $F$ is a quasiregular mapping of transcendental type on $\mathbb{R}^{3}$.

It remains to prove the claim that $F$ is independent of the choice of the inverse branch of $Z$. To this end, let $x \in \mathbb{R}^{3} \backslash\{0\}$ and suppose that $\psi \neq \phi$ is a different inverse branch of $Z$ defined at $x$. Set $F_{1}:=Z \circ T \circ g \circ T^{-1} \circ \psi$.

By the construction of $Z$, there exist some $n, m \in \mathbb{Z}$ and $p \in\{0,1\}$ such that

$$
\phi(x)=R_{(1,1)}^{p}(\psi(x))+(4 n, 4 m, 0),
$$

where $R_{(1,1)}^{1}=R_{(1,1)}$ and $R_{(1,1)}^{0}$ denotes the identity mapping.

Let $u:=\left(T^{-1} \circ \phi\right)(x)$ and let $v:=\left(T^{-1} \circ \psi\right)(x)$. From equations (2.3.1) and (2.3.5) 
we now get

$$
u=R_{(1,1)}^{p}(\psi(x))+(1,1,0)+(4 n, 4 m, 0)=R_{(2,2)}^{p}(v)+(4 n, 4 m, 0) .
$$

It then follows from properties (G3) and (G4) of $g$ that

$$
g(u)=g\left(R_{(2,2)}^{p}(v)+(4 n, 4 m, 0)\right)=R_{(2,2)}^{p}(g(v))+(4 n, 4 m, 0) .
$$

By appealing to equation (2.3.1), then equation (2.3.6) yields

$$
T(g(u))=g(u)-(1,1,0)=R_{(1,1)}^{p}(g(v)-(1,1,0))+(4 n, 4 m, 0) .
$$

Finally, it follows from equation (2.3.2) and the periodicity of $Z$ that

$$
\begin{aligned}
F(x) & =(Z \circ T)(g(u)) \\
& =Z\left(R_{(1,1)}^{p}(g(v)-(1,1,0))+(4 n, 4 m, 0)\right) \\
& =Z(g(v)-(1,1,0)) \\
& =(Z \circ T)(g(v))=F_{1}(x),
\end{aligned}
$$

therefore the claim follows. This completes the proof of Theorem 2.1.2.

As a remark, since $F$ satisfies equation (2.3.4) then $F$ and $g$ are semi-conjugate to each other by the quasiregular mapping $Z \circ T$. Thus for each $n \in \mathbb{N}$,

$$
F^{n}=Z \circ T \circ g^{n} \circ T^{-1} \circ \phi
$$

Proof of Corollary 2.1.3. Let $F$ be as above and let $\hat{M}_{0}: \mathbb{R}^{3} \rightarrow \hat{\mathbb{R}}^{3}$ be the sensepreserving Möbius map defined using equation (1.2.6), where $d=3$.

As $\hat{M}_{0}$ is a sense-preserving Möbius mapping of $\hat{\mathbb{R}}^{3}$ and $F$ is quasiregular of tran- 
scendental type, then $f:=\hat{M}_{0} \circ F: \mathbb{R}^{3} \rightarrow \hat{\mathbb{R}}^{3}$ is a quasimeromorphic mapping of transcendental type. Further by the definition of $\hat{M}_{0}$ and the fact that $F^{-1}(0)=\{0\}$, then $\mathcal{O}_{f}^{-}(\infty)=\{0, \infty\}$ as required.

\subsection{Modifying the construction of $F$}

To create other quasiregular mappings of transcendental type with a value taken finitely often, we can consider replacing the function $g$ with a quasiregular function of transcendental type $\tilde{g}: \mathbb{R}^{3} \rightarrow \mathbb{R}^{3}$ that satisfies the following properties:

(I) for each $n, m \in \mathbb{Z}$, there exist integers $\alpha$ and $\beta$ such that

$$
\tilde{g}(x+(4 n, 4 m, 0))=\tilde{g}(x)+(4 \alpha, 4 \beta, 0),
$$

(II) $\tilde{g}\left(R_{(2,2)}(x)\right)=R_{(2,2)}(\tilde{g}(x))$, and

(III) for every $M \geq 0$, there exists some $N \geq 0$ such that $\tilde{g}(x) \in \mathbb{H}_{\leq-M}$ whenever $x \in \mathbb{H}_{\leq-N}$.

Let $\tilde{F}: \mathbb{R}^{3} \rightarrow \mathbb{R}^{3}$ be defined by $\tilde{F}(0)=0$ and $\tilde{F}(x)=\left(Z \circ T \circ \tilde{g} \circ T^{-1} \circ \phi\right)(x)$ for all $x \in \mathbb{R}^{3} \backslash\{0\}$, where $\phi$ is an inverse branch of $Z$.

To show that $\tilde{F}$ is continuous at 0 , let $\varepsilon>0$ be given and observe that $x \in \mathbb{H}_{\leq \log (\varepsilon)}$ implies that $(Z \circ T)(x) \in B(0,2 \varepsilon)$. By (III), there exists some $N \geq 0$ such that $\tilde{g}(y) \in \mathbb{H}_{\leq \log (\varepsilon)}$ whenever $y \in \mathbb{H}_{\leq-N}$. Therefore by taking $\delta=\exp (-N) / 2>0$, then $w \in B(0, \delta)$ implies that $\left(T^{-1} \circ \phi\right)(w) \in \mathbb{H}_{\leq-N}$ and the continuity of $\tilde{F}$ on $\mathbb{R}^{3}$ follows as before.

Finally to complete the proof, observe that the only required change in the argument given in Section 2.3.2 will be within equation (2.3.6), as this is the only place where we had applied (G3) and (G4). Instead, applying (I) and (II) of $\tilde{g}$ to 
$u=R_{(2,2)}^{p}(v)+(4 n, 4 m, 0)$ yields

$$
\tilde{g}(u)=\tilde{g}\left(R_{(2,2)}^{p}(v)+(4 n, 4 m, 0)\right)=R_{(2,2)}^{p}(\tilde{g}(v))+(4 \alpha, 4 \beta, 0) .
$$

Now due to equation (2.3.2) and the periodicity of $Z$, the proof follows as before. Therefore $\tilde{F}$ is a well-defined quasiregular mapping of transcendental type that also satisfies

$$
(\tilde{F} \circ(Z \circ T))(x)=((Z \circ T) \circ \tilde{g})(x) \text { for all } x \in \mathbb{R}^{3} \backslash\{0\} \text {. }
$$

From here, composing $\tilde{F}$ with $\hat{M}_{0}$ gives us another quasimeromorphic mapping of transcendental type that satisfies equation (2.1.2).

As before, $\tilde{F}$ and $\tilde{g}$ are semi-conjugate to each other by $Z \circ T$, and the iterates of $\tilde{F}$ and $\tilde{g}$ are related as in equation (2.3.7). Consequently, there is a connection between the dynamics of $\tilde{F}$ and $\tilde{g}$.

Lemma 2.4.1. Let $\tilde{g}$ be a quasiregular mapping satisfying (I) - (III) and let $\tilde{F}:=Z \circ T \circ \tilde{g} \circ T^{-1} \circ \phi$. Then $(Z \circ T)(\mathcal{J}(\tilde{g})) \subset \mathcal{J}(\tilde{F})$.

Proof. Let $x \in(Z \circ T)(\mathcal{J}(\tilde{g}))$ and let $U_{x} \subset \mathbb{R}^{3}$ be an arbitrary open neighbourhood of $x$. Since $Z \circ T$ is continuous and open, then there exists some $y \in \mathcal{J}(\tilde{g})$ and an open neighbourhood $U_{y} \subset \mathbb{R}^{3}$ of $y$ such that $(Z \circ T)(y)=x$ and $(Z \circ T)\left(U_{y}\right) \subset U_{x}$. As $\tilde{F}$ is independent of the choice of inverse branch of $Z$, then choose a branch $\phi$ such that $\left(T^{-1} \circ \phi\right)\left(U_{x}\right) \supset U_{y}$.

As $y \in \mathcal{J}(\tilde{g})$, then by Definition 1.3.4 there exists some set $X \subset \mathbb{R}^{3}$ with cap $(X)=0$ such that

$$
\bigcup_{k=0}^{\infty} \tilde{g}^{k}\left(U_{y}\right) \supset \mathbb{R}^{3} \backslash X
$$

Since $Z \circ T$ is a quasiregular mapping, then it follows from Lemma 1.3.3 that 
$Y:=(Z \circ T)(X) \cup\{0\}$ satisfies $\operatorname{cap}(Y)=0$. As $Z\left(\mathbb{R}^{3}\right)=\mathbb{R}^{3} \backslash\{0\}$, then

$$
(Z \circ T)\left(\bigcup_{k=0}^{\infty} \tilde{g}^{k}\left(U_{y}\right)\right) \supset(Z \circ T)\left(\mathbb{R}^{3}\right) \backslash(Z \circ T)(X)=\mathbb{R}^{3} \backslash Y
$$

Now observe that

$$
\begin{aligned}
\bigcup_{k=0}^{\infty} \tilde{F}^{k}\left(U_{x}\right) & =\bigcup_{k=0}^{\infty}(Z \circ T)\left(\tilde{g}^{k}\left(\left(T^{-1} \circ \phi\right)\left(U_{x}\right)\right)\right) \\
& \supset \bigcup_{k=0}^{\infty}(Z \circ T)\left(\tilde{g}^{k}\left(U_{y}\right)\right) \\
& =(Z \circ T)\left(\bigcup_{k=0}^{\infty} \tilde{g}^{k}\left(U_{y}\right)\right) \supset \mathbb{R}^{3} \backslash Y .
\end{aligned}
$$

As $U_{x}$ was an arbitrary neighbourhood of $x$, then we have that $x \in \mathcal{J}(\tilde{F})$ and hence $(Z \circ T)(\mathcal{J}(\tilde{g})) \subset \mathcal{J}(\tilde{F})$.

\subsection{Dynamics of a family of quasiregular maps}

Let $F$ and $g$ be the quasiregular mappings from Section 2.3. Then an example of an infinite family of quasiregular mappings satisfying equation (2.1.1) would be $\mathcal{F}_{\lambda_{0}}:=\left\{\lambda F: 0<\lambda<\lambda_{0}\right\}$. These mappings can be constructed by considering the quasiregular maps $g_{t}: \mathbb{R}^{3} \rightarrow \mathbb{R}^{3}$, which for each $t \in \mathbb{R}$ are defined by

$$
g_{t}(x)=g(x)+(0,0, t)
$$

It is easy to see that for all $t \in \mathbb{R}$, then $g_{t}$ satisfy (I) - (III). It follows from equation (2.3.3) that for all $\lambda>0$ and any inverse branch $\phi$ of $Z$,

$$
\lambda F=Z \circ T \circ g_{\log \lambda} \circ T^{-1} \circ \phi .
$$


The aim of this section is to establish some dynamical results for the family $\mathcal{F}_{\lambda_{0}}$ when $\lambda_{0}>0$ is sufficiently small, culminating in Proposition 2.5.3 below. The dynamics of some quasimeromorphic mappings associated with $\mathcal{F}_{1}$ can also be considered, yielding Lemma 2.5.4 below; this together with Proposition 2.5.3 will prove Theorem 2.1.4.

Firstly, we shall state some properties of $g_{\log \lambda}$ for sufficiently small $\lambda>0$ by Nicks and Sixsmith from [69, Section 7]. This will be summarised below in the following lemma, recalling the definition of the quasi-Fatou set.

Lemma 2.5.1. Let $L>1$ be the constant from property (G2) of $g$. Then there exists a constant $0<C<1$ such that whenever $0<\lambda \leq C$, then the following holds.

(i) $g_{\log \lambda}\left(\mathbb{H}_{\leq L}\right) \subset \mathbb{H}_{<0}$.

(ii) $\mathcal{Q F}\left(g_{\log \lambda}\right)$ consists of a single connected domain containing the lower halfspace $\mathbb{H}_{<0}$, in which all iterates of $g_{\log \lambda}$ tend to infinity locally uniformly. In particular, for every $x \in \mathcal{Q F}\left(g_{\log \lambda}\right)$ there exists some $k \in \mathbb{N}$ such that $g_{\log \lambda}^{k}(x) \in \mathbb{H}_{<0}$.

(iii) The Julia set $\mathcal{J}\left(g_{\log \lambda}\right)$ contains the lines

$$
\left\{\left(4 n+c, 4 m+c, x_{3}\right): n, m \in \mathbb{Z}, c \in\{0,2\}, x_{3}>\log \left(L^{\prime}\right)\right\}
$$

where $L^{\prime}=\max \{\log (1 / \lambda), \exp (L)\}>1$.

By using the semi-conjugacy within equation (2.5.2), we shall first give a result relating the quasi-Fatou set of $g_{\log \lambda}$ to the attracting basin of $\lambda F$ at 0 . Here, for a general mapping $f: \mathbb{R}^{3} \rightarrow \mathbb{R}^{3}$, the attracting basin of 0 with respect to $f$ is defined by $\mathcal{A}_{f}(0):=\left\{x \in \mathbb{R}^{3}: f^{n}(x) \rightarrow 0\right.$ as $\left.n \rightarrow \infty\right\}$. It should be noted that by definition, $\mathcal{A}_{f}(0)$ is completely invariant under $f$. 
Lemma 2.5.2. Let $0<\lambda \leq C$, where $0<C<1$ is the constant from Lemma 2.5.1. Then $(Z \circ T)\left(\mathcal{Q F}\left(g_{\log \lambda}\right)\right) \subset \mathcal{A}_{\lambda F}(0)$.

Proof. Let $x \in(Z \circ T)\left(\mathcal{Q F}\left(g_{\log \lambda}\right)\right)$, so there exists some $y \in \mathcal{Q F}\left(g_{\log \lambda}\right)$ such that $(Z \circ T)(y)=x$. As $\lambda \leq C$, then by Lemma 2.5.1(ii) there exists some $k \in \mathbb{N}$ such that $g_{\log \lambda}^{k}(y) \in \mathbb{H}_{<0}$. Now by choosing $\phi$ such that $\phi(x)=T(y)$, then using equation (2.5.2) and (G1) we get that for all $n \in \mathbb{N}$,

$$
(\lambda F)^{n+k}(x)=\left(Z \circ T \circ g_{\log \lambda}^{n} \circ g_{\log \lambda}^{k} \circ T^{-1} \circ \phi\right)(x)=\left(Z \circ T \circ g_{\log \lambda^{n}}\right)\left(g_{\log \lambda}^{k}(y)\right) .
$$

It follows from equation (2.3.3) that

$$
\left(Z \circ T \circ g_{\log \lambda^{n}}\right)\left(g_{\log \lambda}^{k}(y)\right)=\lambda^{n}(Z \circ T)\left(g_{\log \lambda}^{k}(y)\right) \rightarrow 0 \text { as } n \rightarrow \infty
$$

hence $x \in \mathcal{A}_{\lambda F}(0)$ as required.

By using the above lemma, we can establish a strong relationship between the Julia sets and quasi-Fatou sets of $g_{\log \lambda}$ and $\lambda F$ as follows.

Proposition 2.5.3. Let $L>1$ be the constant from property (G2) of $g$ and let $0<\lambda \leq C$, where $0<C<1$ is the constant from Lemma 2.5.1. Then

(i) $\mathcal{J}(\lambda F)=(Z \circ T)\left(\mathcal{J}\left(g_{\log \lambda}\right)\right)$ contains the half-rays $\left\{t(1,1,0):|t|>L^{\prime}\right\}$, where $L^{\prime}=\max \{\log (1 / \lambda), \exp (L)\}>1$, and

(ii) $\mathcal{Q F}(\lambda F)=\mathcal{A}_{\lambda F}(0)=(Z \circ T)\left(\mathcal{Q F}\left(g_{\log \lambda}\right)\right) \cup\{0\}$ is connected and contains $B(0,1 / 2)$.

Proof. Firstly, recall that $F$ is the identity on $B(0,1 / 2)$. It immediately follows that for all $0<\lambda<1$, then $B(0,1 / 2) \subset \mathcal{A}_{\lambda F}(0) \cap \mathcal{Q F}(\lambda F)$. As $\mathcal{A}_{\lambda F}(0)$ and $\mathcal{Q F}(\lambda F)$ 
are completely invariant, then

$$
\mathcal{A}_{\lambda F}(0) \subset \mathcal{Q F}(\lambda F)
$$

To prove that $\mathcal{J}(\lambda F)=(Z \circ T)\left(\mathcal{J}\left(g_{\log \lambda}\right)\right)$, observe that from Lemma 2.4.1 we have $(Z \circ T)\left(\mathcal{J}\left(g_{\log \lambda}\right)\right) \subset \mathcal{J}(\lambda F)$. For the reverse inclusion, let $x \in \mathcal{J}(\lambda F)$. As $x \neq 0$, then there exists some $y \in \mathbb{R}^{3}$ that $(Z \circ T)(y)=x$. It then follows from Lemma 2.5.2 and equation (2.5.3) that $y \notin \mathcal{Q F}\left(g_{\log \lambda}\right)$. Therefore $y \in \mathcal{J}\left(g_{\log \lambda}\right)$, as required.

Using Lemma 2.5.1(iii), the first part of (i) now implies

$$
\left\{t(1,1,0):|t|>L^{\prime}\right\} \subset \mathcal{J}(\lambda F)
$$

completing the proof of (i).

To prove (ii), note that from (i) we have $\mathcal{Q F}(\lambda F) \subset(Z \circ T)\left(\mathcal{Q F}\left(g_{\log \lambda}\right)\right) \cup\{0\}$, since $(Z \circ T)\left(\mathbb{R}^{3}\right)=\mathbb{R}^{3} \backslash\{0\}$. Further from Lemma 2.5.2 and equation (2.5.3),

$$
\mathcal{Q F}(\lambda F) \subset(Z \circ T)\left(\mathcal{Q F}\left(g_{\log \lambda}\right)\right) \cup\{0\} \subset \mathcal{A}_{\lambda F}(0) \subset \mathcal{Q F}(\lambda F)
$$

thus equality is attained. Further, since $\mathcal{Q} \mathcal{F}\left(g_{\log \lambda}\right)$ is a single connected domain and $B(0,1 / 2) \subset \mathcal{Q F}(\lambda F)$, then $\mathcal{Q F}(\lambda F)$ is connected, completing the proof.

Define $f_{\lambda}:=\hat{M}_{0} \circ \lambda F$, where $\hat{M}_{0}$ is the Möbius map defined from equation (1.2.6), and recall the definition of the bungee set $B U(f)$ from Section 1.4. By considering the behaviour of $\lambda F$ and $\hat{M}_{0}$, we can explicitly locate points in $B U\left(f_{\lambda}\right)$. In particular, we can explicitly show that the half-rays from Proposition 2.5.3(i) are in this set. 
Lemma 2.5.4. Let $0<\lambda<1$, let $L>1$ be the constant from property (G2) of $g$ and let $f_{\lambda}=\hat{M}_{0} \circ \lambda F$. Then

$$
\left\{t(1,1,0):|t|>L^{\prime}\right\} \cup\left\{t(1,1,0): 0<|t|<1 /\left(2 L^{\prime}\right)\right\} \subset B U\left(f_{\lambda}\right)
$$

where $L^{\prime}=\max \{\log (1 / \lambda), \exp (L)\}$.

Proof. Let $0<\lambda<1$ and let $L>1$ be the constant from property (G2) of $g$. Now consider the behaviour of $\lambda F$ on the line $\{t(1,1,0): t \in \mathbb{R}\}$. Indeed by the properties of $Z, g_{\log \lambda}$ and equation (2.5.2), a direct calculation yields

$$
(\lambda F)(\alpha(1,1,0))= \begin{cases}\lambda \exp (|\alpha|) \alpha(1,1,0) & \text { if }|\alpha|>\exp (L) \\ \lambda \alpha(1,1,0) & \text { if }|\alpha|<1\end{cases}
$$

It follows that as $n \rightarrow \infty$, then $(\lambda F)^{n}(\alpha(1,1,0)) \rightarrow 0$ when $|\alpha|<1$, while $(\lambda F)^{n}(\alpha(1,1,0)) \rightarrow \infty$ when $|\alpha|>\max \{\log (1 / \lambda), \exp (L)\}$.

Next note that for any $\alpha \in \mathbb{R}$ we have

$$
\hat{M}_{0}(\alpha(1,1,0))=\frac{1}{2 \alpha}(1,1,0) .
$$

Combining this with equation (2.5.4), it follows that

$$
f_{\lambda}(\alpha(1,1,0))= \begin{cases}\frac{1}{2 \lambda \exp (|\alpha|) \alpha}(1,1,0) & \text { if }|\alpha|>\exp (L) \\ \frac{1}{2 \lambda \alpha}(1,1,0) & \text { if }|\alpha|<1\end{cases}
$$

Now observe that when $|\alpha|>\max \{\log (1 / \lambda), \exp (L)\}$, then

$$
|1 /(2 \lambda \exp (|\alpha|) \alpha)|<|1 /(2 \lambda \exp (\log (1 / \lambda)) \exp (L))|=|1 /(2 \exp (L))|<1
$$


Further when $|\alpha|<1 /(2 \max \{\log (1 / \lambda), \exp (L)\})<1 /(2 \exp (L) \lambda)$, then it follows that

$$
|1 /(2 \lambda \alpha)|>\exp (L)
$$

Finally by considering the even and odd iterates of $f_{\lambda}$ in light of equations (2.5.5), (2.5.6) and (2.5.7), the result follows. 


\section{A growth result for quasiregular mappings near an essential singularity}

In this chapter, we shall aim to generalise a growth result for quasiregular mappings of transcendental type to mappings that are quasiregular near an essential singularity. Although the original proof given by Bergweiler in [10] cannot be applied to these mappings, we shall show it is possible to significantly adapt the ideas to obtain the proof in the new setting. This theorem, and a subsequent useful corollary, will find several important applications throughout the remainder of this thesis that facilitate the iterative study of quasimeromorphic mappings of transcendental type with at least one pole.

Much of the content forming this chapter can be found in sections 2 and 3 of [108].

\subsection{Introduction}

When establishing covering results for a transcendental entire function $f: \mathbb{C} \rightarrow \mathbb{C}$, an important tool to consider is the maximum modulus, defined by

$$
M(r, f)=\max \{|f(z)|:|z|=r\}
$$


where $r>0$. As $f$ is continuous and open, then given any $r>0$ there exists a point $z^{*} \in \mathbb{C}$ such that $\left|z^{*}\right|=r$ and $\left|f\left(z^{*}\right)\right|=M(r, f)$. Further, it follows from the maximum principle that $M(r, f)$ is a strictly increasing function of $r$.

A well-known property of the maximum modulus for transcendental entire functions $f$ is that $\log (M(r, f))$ is a convex function over $\log (r)$. As $f$ has an essential singularity at infinity, then given any $\lambda>1$ it follows that

$$
\lim _{r \rightarrow \infty} \frac{M(\lambda r, f)}{M(r, f)}=\infty
$$

The above property has found numerous applications within covering results; for example see $[16,89]$.

Since quasiregular mappings of transcendental type are continuous, open and have an essential singularity at infinity, then we can naturally extend the maximum modulus and some of its properties to the higher-dimensional setting. However if $f: \mathbb{R}^{d} \rightarrow \mathbb{R}^{d}$ is a quasiregular mapping then $\log (M(r, f))$ may not necessarily be convex with respect to $\log (r)$; this result is due to Bergweiler, Drasin and Fletcher [13], who used unpublished ideas of Nicks to construct a quasiregular mapping $f$ where $\log (M(r, f)) / \log (r)$ is decreasing over a collection of intervals.

Nonetheless, Bergweiler did manage to extend equation (3.1.1) to the quasiregular setting in [10], yielding the following growth result for quasiregular mappings of transcendental type.

Lemma 3.1.1. Let $f: \mathbb{R}^{d} \rightarrow \mathbb{R}^{d}$ be a quasiregular mapping of transcendental type, and let $\lambda>1$. Then

$$
\lim _{r \rightarrow \infty} \frac{M(\lambda r, f)}{M(r, f)}=\infty
$$

The proof of Lemma 3.1.1 relies on covering the whole domain $\mathbb{R}^{d}$ by increasingly large balls to achieve a contradiction via the topological degree (see Section 3.1.1 below). Unfortunately, for this reason it cannot be applied to mappings that are only 
quasiregular in a neighbourhood of an essential singularity, such as the mapping $f$ from Theorem 2.1.2 restricted to $A(1, \infty)$.

Instead by adapting the proof structure used by Bergweiler, we can successfully extend Lemma 3.1.1 to the case where the mapping is quasiregular in a neighbourhood of an essential singularity. This is stated as follows.

Theorem 3.1.2. Let $R>0$, let $f: A(R, \infty) \rightarrow \mathbb{R}^{d}$ be a quasiregular map with an essential singularity at infinity, and let $\lambda>1$. Then

$$
\lim _{r \rightarrow \infty} \frac{M(\lambda r, f)}{M(r, f)}=\infty
$$

As an immediate consequence of Theorem 3.1.2, we get the following useful corollary.

Corollary 3.1.3. Let $R>0$ and $f: A(R, \infty) \rightarrow \mathbb{R}^{d}$ be a quasiregular map with an essential singularity at infinity. Then

$$
\lim _{r \rightarrow \infty} \frac{\log M(r, f)}{\log (r)}=\infty
$$

Before continuing to the proof of Theorem 3.1.2, we shall make the following important remark. Namely, Theorem 3.1.2 can be applied within the proof of [67, Lemma 2.6] to rectify an omission there, where it was claimed that the proof of a statement like Theorem 3.1.2 is similar to that of Lemma 3.1.1. However, the mappings studied in [67] form a higher-dimensional analogue of holomorphic self-maps of the punctured plane $\mathbb{C}^{*}$ (see Section 4.1.1); such mappings are only quasiregular in a neighbourhood of an essential singularity. As a result of rectifying this omission and recovering the Fatou-Julia theory of such mappings in [67], it has been possible to establish the new iterative theory of quasimeromorphic mappings of transcendental type with at least one pole. 


\subsubsection{Topological degree and a removability lemma}

Let $f: G \rightarrow \mathbb{R}^{d}$ be a quasiregular mapping, $D \subset G$ be an open set with $\bar{D} \subset G$ compact, and let $y \in \mathbb{R}^{d} \backslash f(\partial D)$. Firstly, for $x \in G$, we define the local (topological) index of $f$ at $x$, denoted by $i(x, f)$, as

$$
i(x, f):=\inf \left\{\sup \left\{\operatorname{card}\left(f^{-1}(w) \cap U_{x}\right): w \in \mathbb{R}^{d}\right\}\right\}
$$

where the infimum is taken over all the neighbourhoods $U_{x} \subset G$ of $x$.

From here we define the topological degree of $f$ at $y$ over $D$, denoted $\mu(y, f, D)$, as

$$
\mu(y, f, D)=\sum_{x \in f^{-1}(y) \cap D} i(x, f),
$$

which informally counts the number of preimages of $y$ in $D$ including multiplicity. For quasiregular mappings, the topological degree has many useful properties which will be summarised below without proof (See [76, Section II.2.3] and [87, Proposition I.4.4]). Here, for $r \in \mathbb{R}$, we use the notation $r D:=\{r x: x \in D\}$.

Theorem 3.1.4. Let $f: G \rightarrow \mathbb{R}^{d}$ be a quasiregular mapping and let $D \subset \mathbb{R}^{d}$ be a bounded open set with $\bar{D} \subset G$. Then the following hold.

(i) If $x, y \notin f(\partial D)$ are in the same connected component of $\mathbb{R}^{d} \backslash f(\partial D)$, then $\mu(x, f, D)=\mu(y, f, D)$.

(ii) If $y \notin f(\partial D), X_{1}, X_{2}, \ldots, X_{n}$ are disjoint sets and if $D \cap f^{-1}(y) \subset \cup_{i} X_{i} \subset D$, then

$$
\mu(y, f, D)=\sum_{i=1}^{n} \mu\left(y, f, X_{i}\right) \text { (when defined). }
$$

(iii) If $y \notin f(\partial D)$ and $g: H \rightarrow \mathbb{R}^{d}$ is a quasiregular mapping with $\bar{D} \subset H$ such that $\max \{|f(x)-g(x)|: x \in \partial D\}<\min \{|f(x)-y|: x \in \partial D\}$, then $\mu(y, f, D)=\mu(y, g, D)$. 
(iv) If $\alpha, \beta>0$ and $\alpha y \notin f(\partial D)$, then

$$
\mu(\alpha y, f, D)=\mu\left(y, F, D^{\prime}\right)
$$

where $D^{\prime}=(1 / \beta) D$ and $F: \Omega \rightarrow \mathbb{R}^{d}$ is a quasiregular mapping with $\Omega \supset \overline{D^{\prime}}$, defined by $F(x)=(1 / \alpha) f(\beta x)$.

Another important theorem is a sufficient condition for when a quasiregular mapping can be extended over isolated points. The following theorem follows from a result first established by Callendar [23], which was later generalised by Martio, Rickman and Väisälä [59].

Theorem 3.1.5. Let $D \subset \hat{\mathbb{R}}^{d}$ be a domain, $E \subset D$ be a finite set of points and $f: D \backslash E \rightarrow \mathbb{R}^{d}$ be a bounded $K$-quasiregular mapping. Then $f$ can be extended to a K-quasiregular mapping on all of $D$.

\subsection{Proof of Theorem 3.1.2}

Before we begin the proof of Theorem 3.1.2, we will first note the following fact about the maximum modulus for quasiregular mappings defined in a neighbourhood of an essential singularity; this follows from the maximum modulus principle and an application of Theorem 1.2.6. We shall include the proof here for completeness.

Lemma 3.2.1. Let $R>0$ and let $f: A(R, \infty) \rightarrow \mathbb{R}^{d}$ be a $K$-quasiregular mapping with an essential singularity at infinity. Then there exists $R^{\prime} \geq R$ such that $M(r, f)$ is a strictly increasing function for $r>R^{\prime}$.

Proof. Firstly by increasing $R$ if necessary, we may assume that $f$ is continuous on $\overline{A(R, \infty)} \backslash\{\infty\}$. Now note that $g(r):=M(r, f)$ is a non-constant continuous real function on $(R, \infty)$, as $f$ is open and continuous. From the openness of $f$ and the 
maximium principle, it follows that $g$ does not have a local maximum and hence is also non-constant for all intervals in $[R, \infty)$.

It now follows that $g$ must either be strictly increasing or strictly decreasing on all points in $[R, \infty)$, or $g$ must have exactly one minimum value. If $g$ has one minimum, then we set $R^{\prime} \in[R, \infty)$ to be the value that attains it. If $g$ is strictly decreasing everywhere, then $f$ is bounded above by $M(R, f)$. By Theorem 3.1.5, $f$ has a limit at infinity, which contradicts the fact that infinity is an essential singularity of $f$. The result then follows.

Using the above, we now aim to prove Theorem 3.1.2. Firstly, let $R>0$ be sufficiently large such that $f: A(R, \infty) \rightarrow \mathbb{R}^{d}$ is a $K$-quasiregular mapping with an essential singularity at infinity, and $M(r, f)$ is a strictly increasing function for $r>R$.

Now let $\lambda>1$ be given and suppose for a contradiction to Theorem 3.1.2 that there exists some constant $L>1$ and some real sequence $r_{n} \rightarrow \infty$ such that $M\left(\lambda r_{n}, f\right) \leq L M\left(r_{n}, f\right)$. By taking a subsequence and then starting from large enough $n$, we may assume that $\left(r_{n}\right)$ is a strictly increasing sequence with $r_{1}>R$. Define a new sequence $\left(f_{n}\right)$ by

$$
f_{n}(x):=\frac{f\left(r_{n} x\right)}{M\left(r_{n}, f\right)}
$$

For each $N \in \mathbb{N}$, let $A_{N}:=A\left(R / r_{N}, \lambda\right)$. Now for all $n \geq N, f_{n}$ is well-defined and $K$-quasiregular on $A_{N}$.

Lemma 3.2.2. There exists a map $h$ defined on $B(0, \lambda) \backslash\{0\}$, which is either a bounded $K$-quasiregular mapping or a constant function, and a subsequence of $\left(f_{n}\right)$ that converges to h locally uniformly on $B(0, \lambda) \backslash\{0\}$. 
Proof. Observe that for each $n \geq N$ and $x \in A_{N}$,

$$
\left|f_{n}(x)\right| \leq \frac{M\left(r_{n}|x|, f\right)}{M\left(r_{n}, f\right)} \leq \frac{M\left(\lambda r_{n}, f\right)}{M\left(r_{n}, f\right)} \leq L
$$

As $L$ is not dependent on $N$, then $f_{n}$ is uniformly bounded on $A_{N}$ for all $n \geq N$. By Lemma 1.3.1, $\mathcal{F}_{N}:=\left\{f_{n}: n \geq N\right\}$ is a normal family on $A_{N}$ for each $N \in \mathbb{N}$. In particular, for the sequence $\left(f_{n}\right) \subset \mathcal{F}_{1}$ there exists a subsequence $\left(f_{1, k}\right)_{k=1}^{\infty} \subset\left(f_{n}\right)$ such that $\left(f_{1, k}\right)$ converges locally uniformly on $A_{1}$. Discarding the first term if necessary, we may assume that $\left(f_{1, k}\right) \subset \mathcal{F}_{2}$ so the subsequence is defined and uniformly bounded on $A_{2}$. Thus there exists a subsequence $\left(f_{2, k}\right)_{k=1}^{\infty} \subset\left(f_{1, k}\right)$ such that $\left(f_{2, k}\right)$ converges locally uniformly on $A_{2}$.

By repeating this process, we build a sequence of subsequences $\left(f_{1, k}\right),\left(f_{2, k}\right), \ldots$, such that $\left(f_{i, k}\right) \supset\left(f_{i+1, k}\right)$ for all $i \in \mathbb{N}$ and $\left(f_{i, k}\right)$ converges locally uniformly on $A_{i}$. Now consider the sequence $\left(f_{k, k}\right)$ and observe that $\left(f_{k, k}\right)_{k \geq i}$ is a subsequence of each $\left(f_{i, k}\right)$ with $i \in \mathbb{N}$ by construction. This means that the pointwise limit function

$$
h(w):=\lim _{k \rightarrow \infty} f_{k, k}(w)
$$

exists on $B(0, \lambda) \backslash\{0\}$.

Let $D \subset B(0, \lambda) \backslash\{0\}$ be a compact set. Then there exists some $N \in \mathbb{N}$ such that $D \subset A_{N}$ and $\left(f_{k, k}\right)_{k \geq N}$ is defined on $D$.

Now by construction, $\left(f_{N, k}\right)$ converges uniformly on $D$. As $\left(f_{k, k}\right)_{k \geq N}$ is a subsequence of $\left(f_{N, k}\right)$, then from equation (3.2.3) we have that $f_{k, k} \rightarrow h$ uniformly on $D$. Further, since $\left(f_{k, k}\right)_{k \geq N}$ is a sequence of $K$-quasiregular mappings on $D$, then $h$ is either $K$-quasiregular or constant on $D$. Finally since $D$ was arbitrary, then $f_{k, k} \rightarrow h$ locally uniformly on $B(0, \lambda) \backslash\{0\}$ and $h$ is either $K$-quasiregular or constant on $B(0, \lambda) \backslash\{0\}$.

It remains to note that from equation (3.2.2), for all $x \in B(0, \lambda) \backslash\{0\}$ we have 
$|h(x)| \leq L$, thus $h$ is bounded as required.

By discarding terms and relabelling, we may assume that $f_{n} \rightarrow h$ locally uniformly on $B(0, \lambda) \backslash\{0\}$. Using Theorem 3.1.5, we can extend $h$ to either a $K$-quasiregular mapping or a constant mapping defined on $B(0, \lambda)$. By relabelling, let this extended map be $h$.

Before showing that $h(0)=0$, we make an observation. For each $n \in \mathbb{N}$, let $x_{n} \in S(1)$ be such that $\left|f\left(r_{n} x_{n}\right)\right|=M\left(r_{n}, f\right)$. As $S(1)$ is compact, then there exists a subsequence $\left(x_{n_{t}}\right)$ of $\left(x_{n}\right)$ that converges to some point $\tilde{x} \in S(1)$. Since $f_{n} \rightarrow h$ locally uniformly on $B(0, \lambda) \backslash\{0\}$, then it follows that $f_{n_{t}}\left(x_{n_{t}}\right) \rightarrow h(\tilde{x})$ as $t \rightarrow \infty$. Therefore, $|h(\tilde{x})|=1$ for such $\tilde{x} \in S(1)$.

Lemma 3.2.3. Let $h$ be as above. Then $h(0)=0$, so $h$ is a K-quasiregular mapping.

Proof. Suppose that $|h(0)|=\zeta \neq 0$. Let $T>4 / \zeta,\left(z_{m}\right) \subset B(0, \lambda) \backslash\{0\}$ be a sequence such that $z_{m} \rightarrow 0$ as $m \rightarrow \infty$, and define $S_{m}:=S\left(\left|z_{m}\right|\right)$ for each $m \in \mathbb{N}$. As $h$ is a continuous function, then there exists some $\delta>0$ such that $|h(x)-h(0)|<1 / 2 T$ whenever $|x|<\delta$. In particular, there exists an $M \in \mathbb{N}$ such that $\left|z_{m}\right|<\delta$ when $m \geq M$. Hence for all $x \in S_{m}$, we have $|h(x)-h(0)|<1 / 2 T$ whenever $m \geq M$.

Now as $f_{n} \rightarrow h$ locally uniformly on $B(0, \lambda) \backslash\{0\}$, there exists some $N_{M} \in \mathbb{N}$ such that for all $x \in S_{M}$, we have $\left|f_{n}(x)-h(x)\right|<1 / 2 T$ when $n \geq N_{M}$. Therefore, for every $x \in S_{M}$,

$$
\left|f_{n}(x)-h(0)\right| \leq\left|f_{n}(x)-h(x)\right|+|h(x)-h(0)|<\frac{1}{2 T}+\frac{1}{2 T}=\frac{1}{T},
$$

whenever $n \geq N_{M}$. Fix such an $n$.

Since $M\left(r_{k}, f\right) \rightarrow \infty$ as $k \rightarrow \infty$, then there must exist some $t \in \mathbb{N}$ such that 
$M\left(r_{n+t}, f\right)>2 M\left(r_{n}, f\right)$. Now consider $V:=A\left(r_{n}\left|z_{M}\right|, r_{n+t}\left|z_{M}\right|\right)$.

As $n \geq N_{M}$ then from equation (3.2.4),

$$
\begin{aligned}
f\left(r_{n} S_{M}\right) & =M\left(r_{n}, f\right) f_{n}\left(S_{M}\right) \subset B\left(M\left(r_{n}, f\right) h(0), \frac{M\left(r_{n}, f\right)}{T}\right)=: B_{n}, \text { and } \\
f\left(r_{n+t} S_{M}\right) & =M\left(r_{n+t}, f\right) f_{n+t}\left(S_{M}\right) \\
\subset B\left(M\left(r_{n+t}, f\right) h(0), \frac{M\left(r_{n+t}, f\right)}{T}\right) & =: B_{n+t} .
\end{aligned}
$$

Since $M\left(r_{n+t}, f\right)>2 M\left(r_{n}, f\right)$ and $T \zeta>4$, it follows that $\bar{B}_{n} \cap \bar{B}_{n+t}=\varnothing$.

As $f$ is continuous and open, then $f(V)$ is an open path-connected set. Now there exist $x \in f(V) \cap B_{n}, y \in f(V) \cap B_{n+t}$ and a continuous path $\beta:[0,1] \rightarrow f(V)$ with endpoints $x$ and $y$.

Since $\bar{B}_{n}$ and $\bar{B}_{n+t}$ are disjoint, then there must exist some $c \in(0,1)$ such that $\beta(c) \in f(V) \backslash\left(B_{n} \cup B_{n+t}\right)$. However, as $f$ is open, then $\partial f(V) \subset f(\partial V) \subset B_{n} \cup B_{n+t}$, so $f(V)$ must be unbounded. This contradicts the fact that $f$ is continuous on $\bar{V}$.

Now by Theorem 1.2.6, there exists some $a \in \mathbb{R}^{d}$ such that $f$ takes the value $a$ infinitely often. Without loss of generality we may assume that $a=0$, else we can consider instead the function $f(x+a)-a$ rather than $f$. We aim to get a contradiction using the topological degree of $f$ and $h$.

Let $t_{2} \in(0, \lambda)$ be such that $h(x) \neq 0$ for all $x \in S\left(t_{2}\right)$, and let $F:=\min \{|h(x)|$ : $\left.x \in S\left(t_{2}\right)\right\}>0$. Since $h(0)=0$ and $h$ is continuous at 0 , then we can choose some $t_{1} \in\left(0, t_{2}\right)$ such that $P:=M\left(t_{1}, h\right)<F / 4$. Next, set $U:=A\left(t_{1}, t_{2}\right)$ so we have $\bar{U} \subset B(0, \lambda) \backslash\{0\}$. Using this spherical shell, we will show that for all $y \in A(2 P, F / 2)$, the topological degrees of $f_{n}$ and $h$ at $y$ over $U$ agree for large $n$.

Lemma 3.2.4. Let $f_{n}$ be defined as in equation (3.2.1) and let $h$ be defined as in Lemma 3.2.3. Then there exists some $N \in \mathbb{N}$ such that for all $y \in A(2 P, F / 2)$, we 
have $\mu\left(y, f_{n}, U\right)=\mu(y, h, U)$ whenever $n \geq N$.

Proof. As $f_{n} \rightarrow h$ uniformly on compact subsets of $B(0, \lambda) \backslash\{0\}$, then there exists $N \in \mathbb{N}$ such that

$$
\sup \left\{\left|f_{n}(x)-h(x)\right|: x \in \partial U\right\} \leq \sup \left\{\left|f_{n}(x)-h(x)\right|: x \in \bar{U}\right\}<P,
$$

whenever $n \geq N$. In particular, for all $n \geq N$ and for all $x \in \partial U$, we have ||$f_{n}(x)|-| h(x) \| \leq P$. It follows that whenever $n \geq N$, then

$$
M\left(t_{1}, f_{n}\right) \leq M\left(t_{1}, h\right)+P=2 P,
$$

and

$$
\min \left\{\left|f_{n}(x)\right|: x \in S\left(t_{2}\right)\right\}>\min \left\{|h(x)|: x \in S\left(t_{2}\right)\right\}-\frac{F}{2}=F-\frac{F}{2}=\frac{F}{2} .
$$

Now, for all $n \geq N$ we have that $A(2 P, F / 2) \subset f_{n}(U)$ since the $f_{n}$ are open and continuous. In addition, $A(2 P, F / 2) \subset h(U)$ by construction.

Let $y \in A(2 P, F / 2)$. Then for all $x \in \partial U$ and $n \geq N$, we have $f_{n}(x) \neq y$ and $h(x) \neq y$. Thus from equations (3.2.6) and (3.2.7), whenever $n \geq N$ we have

$$
\begin{aligned}
\min \{|h(x)-y|: x \in \partial U\} & >\min \left\{2 P-M\left(t_{1}, h\right), \min \left\{|h(x)|: x \in S\left(t_{2}\right)\right\}-\frac{F}{2}\right\} \\
& =\min \left\{P, \frac{F}{2}\right\}=P .
\end{aligned}
$$

Therefore by Theorem 3.1.4(i),(iii) and equation (3.2.5), it follows that $\mu\left(y, f_{n}, U\right)=$ $\mu(y, h, U)$ for all $y \in A(2 P, F / 2)$ whenever $n \geq N$.

Fix some $y_{0} \in A(2 P, F / 2)$. As $h$ is a discrete mapping, then $h^{-1}\left(y_{0}\right) \cap U$ is a finite set and so

$$
d:=\mu\left(y_{0}, h, U\right)<\infty .
$$


Using equation (3.2.8) and Lemma 3.2.4, we shall now aim for a contradiction by considering the behaviour of $\mu\left(y_{0}, f_{n}, U\right)$ as $n \rightarrow \infty$.

For $n \geq N$, define $d_{n}=\mu\left(y_{0}, f_{n}, U\right), y_{n}=M\left(r_{n}, f\right) y_{0}$ and $U_{n}=A\left(r_{n} t_{1}, r_{n} t_{2}\right)=r_{n} U$.

Now observe that for each $n \geq N$, we have $y_{n} \notin f\left(\partial U_{n}\right)$. It then follows by Theorem 3.1.4(iv) and equation (3.2.1) that for each $n \geq N$,

$$
d_{n}=\mu\left(y_{0}, f_{n}, U\right)=\mu\left(M\left(r_{n}, f\right) y_{0}, f, U_{n}\right)=\mu\left(y_{n}, f, U_{n}\right) .
$$

Lemma 3.2.5. Let $d_{n}$ be as in equation (3.2.9). Then $d_{n} \rightarrow \infty$ as $n \rightarrow \infty$.

Proof. Fix some $n \geq N$ and consider $d_{n}=\mu\left(y_{n}, f, U_{n}\right)$ and $d_{n+1}=\mu\left(y_{n+1}, f, U_{n+1}\right)$.

First note that from equations (3.2.1) and (3.2.6) we have

$$
M\left(t_{1}, f_{n+1}\right)=\frac{M\left(r_{n+1} t_{1}, f\right)}{M\left(r_{n+1}, f\right)} \leq 2 P .
$$

Now since $\left|y_{n+1}\right|>2 P M\left(r_{n+1}, f\right) \geq M\left(r_{n+1} t_{1}, f\right)$, it follows that

$$
\mu\left(y_{n+1}, f, A\left(r_{n} t_{1}, r_{n+1} t_{1}\right)\right)=0 .
$$

Next, as $\left|y_{n}\right|,\left|y_{n+1}\right| \in\left(2 P M\left(r_{n}, f\right),(F / 2) M\left(r_{n+1}, f\right)\right)$, then Theorem 3.1.4(i) gives,

$$
\mu\left(y_{n}, f, A\left(r_{n} t_{1}, r_{n+1} t_{2}\right)\right)=\mu\left(y_{n+1}, f, A\left(r_{n} t_{1}, r_{n+1} t_{2}\right)\right)
$$

Finally, as $\min \left\{\left|f_{n}(x)\right|: x \in S\left(t_{2}\right)\right\}>F / 2$, then

$$
\min \left\{|f(x)|: x \in S\left(r_{n} t_{2}\right)\right\}>\frac{F}{2} M\left(r_{n}, f\right)>\left|y_{n}\right|>0
$$


This means by Theorem 3.1.4(i),

$$
\mu\left(0, f, A\left(r_{n} t_{2}, r_{n+1} t_{2}\right)\right)=\mu\left(y_{n}, f, A\left(r_{n} t_{2}, r_{n+1} t_{2}\right)\right)
$$

Therefore, using equations (3.2.10), (3.2.11), (3.2.12) and Theorem 3.1.4(ii),

$$
\begin{aligned}
d_{n+1} & =d_{n+1}+\mu\left(y_{n+1}, f, A\left(r_{n} t_{1}, r_{n+1} t_{1}\right)\right) \\
& =\mu\left(y_{n+1}, f, A\left(r_{n} t_{1}, r_{n+1} t_{2}\right)\right) \\
& =\mu\left(y_{n}, f, A\left(r_{n} t_{1}, r_{n+1} t_{2}\right)\right) \\
& =\mu\left(y_{n}, f, A\left(r_{n} t_{2}, r_{n+1} t_{2}\right)\right)+d_{n} \\
& =\mu\left(0, f, A\left(r_{n} t_{2}, r_{n+1} t_{2}\right)\right)+d_{n} .
\end{aligned}
$$

Now for all $n \geq N$, by applying equation (3.2.13) finitely many times and using Theorem 3.1.4(ii) again we get that,

$$
d_{n}=\sum_{i=N}^{n-1} \mu\left(0, f, A\left(r_{i} t_{2}, r_{i+1} t_{2}\right)\right)+d_{N}=\mu\left(0, f, A\left(r_{N} t_{2}, r_{n} t_{2}\right)\right)+d_{N} .
$$

Finally as $f$ has infinitely many zeros, then $\mu\left(0, f, A\left(r_{N} t_{2}, r_{n} t_{2}\right)\right) \rightarrow \infty$ as $n \rightarrow \infty$, completing the proof.

A contradiction now follows from Lemma 3.2.4, Lemma 3.2.5 and equation (3.2.8), completing the proof of Theorem 3.1.2. 


\section{$4 \quad$ Julia sets of quasimeromorphic mappings of transcendental type}

The aim of this chapter is to extend the Fatou-Julia theory to the case of quasimeromorphic mappings of transcendental type with at least one pole. We aim to establish a new Julia set definition for these mappings and show that it shares many properties with Julia sets for transcendental meromorphic functions and with the quasiregular analogue of holomorphic self-maps of the punctured plane $\mathbb{C}^{*}$. This will be done through case analysis based on the cardinality of the backward orbit of infinity.

Much of the content forming this chapter can be found in [107].

\subsection{Introduction}

If $f$ is a quasimeromorphic mapping of transcendental type with no poles, then it is precisely an entire quasiregular mapping of transcendental type; the Fatou-Julia theory of these mappings has already been discussed in Section 1.3. Consequently throughout this chapter we shall only consider quasimeromorphic mappings of transcendental type with at least one pole or, in other words, quasimeromorphic 
mappings of transcendental type $f$ such that $\operatorname{card}\left(\mathcal{O}_{f}^{-}(\infty)\right) \geq 2$. We briefly recall that the lower bound of $\operatorname{card}\left(\mathcal{O}_{f}^{-}(\infty)\right)=2$ can be attained, for example, by $f(z)=$ $\exp (z) / z$ in dimension two.

When attempting to define a suitable Julia set for quasimeromorphic mappings of transcendental type with at least one pole, it is important that the set satisfies analogous properties to those found in Theorem 1.1.3. As discussed in Section 1.3, it is therefore unreasonable to directly adapt Definition 1.1.1 to the new setting and define the new Julia set as the complement of the Fatou set. However, as these mappings are related to both transcendental meromorphic functions and to quasiregular mappings of transcendental type, it is reasonable to expect the structure of the new Julia set to share similarities with the Julia sets of those mappings too.

First, recall the Julia set of a transcendental meromorphic function $g: \mathbb{C} \rightarrow \hat{\mathbb{C}}$ defined in Definition 1.1.1. By Theorem 1.1.3(v) and (vi), we can explicitly represent the Julia set $\mathcal{J}(g)$ as

$$
\begin{aligned}
\mathcal{J}(g):= & \left\{z \in \hat{\mathbb{C}} \backslash \overline{\mathcal{O}_{g}^{-}(\infty)}: \operatorname{card}\left(\hat{\mathbb{C}} \backslash \mathcal{O}_{g}^{+}\left(U_{z}\right)\right) \leq 2\right. \text { for all } \\
& \text { neighbourhoods } \left.U_{z} \subset \hat{\mathbb{C}} \backslash \overline{\mathcal{O}_{g}^{-}(\infty)} \text { of } z\right\} \cup \overline{\mathcal{O}_{g}^{-}(\infty)}
\end{aligned}
$$

This representation emphasises the dichotomy that arises as a result of the cardinality of the backward orbit of infinity. This is because for any set $U \subset \hat{\mathbb{C}} \backslash \overline{\mathcal{O}_{g}^{-}(\infty)}$, we have that $\mathcal{O}_{g}^{+}(U)$ is disjoint from $\mathcal{O}_{g}^{-}(\infty)$, and so $\mathcal{O}_{g}^{-}(\infty) \subset \hat{\mathbb{C}} \backslash \mathcal{O}_{g}^{+}(U)$.

By using Picard's theorem, we may condense the above representation to get

$$
\mathcal{J}(g)=\left\{z \in \hat{\mathbb{C}}: \operatorname{card}\left(\hat{\mathbb{C}} \backslash \mathcal{O}_{g}^{+}\left(U_{z}\right)\right) \leq 2 \text { for all neighbourhoods } U_{z} \subset \hat{\mathbb{C}} \text { of } z\right\}
$$

As Example 1.2.4 and Corollary 2.1.3 demonstrate, in the new setting the backward 
orbit of infinity can be finite or infinite. In particular recalling Theorem 1.2.6, if $f$ is a quasimeromorphic mapping of transcendental type with at least one pole, then two cases can arise: either $2 \leq \operatorname{card}\left(\mathcal{O}_{f}^{-}(\infty)\right)<q$ or $\operatorname{card}\left(\mathcal{O}_{f}^{-}(\infty)\right)=\infty$, where $q$ is Rickman's constant.

In light of Conjecture 1.3.6, Definition 1.3.4 and the Julia set defined above, for quasimeromorphic mappings of transcendental type with at least one pole we shall define the Julia set as follows.

Definition 4.1.1. Let $d \geq 2$ and $f: \mathbb{R}^{d} \rightarrow \hat{\mathbb{R}}^{d}$ be a quasimeromorphic mapping of transcendental type with at least one pole. Then the Julia set of $f$ is defined by $\mathcal{J}(f):=\left\{x \in \hat{\mathbb{R}}^{d}: \operatorname{card}\left(\hat{\mathbb{R}}^{d} \backslash \mathcal{O}_{f}^{+}\left(U_{x}\right)\right)<\infty\right.$ for all neighbourhoods $U_{x} \subset \hat{\mathbb{R}}^{d}$ of $\left.x\right\}$.

We immediately remark that analogous to the Julia set for transcendental meromorphic functions, if $f$ is a quasimeromorphic mapping of transcendental type with at least one pole and $\operatorname{card}\left(\mathcal{O}_{f}^{-}(\infty)\right)=\infty$, then $\mathcal{J}(f)=\overline{\mathcal{O}_{f}^{-}(\infty)}$.

To justify that this set is indeed the Julia set for such mappings, we will immediately show that this definition agrees with Definition 1.1.1 for transcendental meromorphic functions with at least one pole. Here, we identify $\mathbb{C}$ with $\mathbb{R}^{2}$ in the usual way.

Theorem 4.1.2. Let $f: \mathbb{C} \rightarrow \hat{\mathbb{C}}$ be a transcendental meromorphic function with at least one pole. Then the classical Julia set of $f$ from Definition 1.1 .1 agrees with Definition 4.1.1.

Proof. Let $\mathcal{F}(f)$ denote the Fatou set of $f$ and, for clarity, let $\mathcal{J}_{\text {mero }}(f):=\hat{\mathbb{C}} \backslash \mathcal{F}(f)$ denote the classical Julia set of $f$ and let $\mathcal{J}(f)$ denote the set from equation (4.1.2). If $x \in \hat{\mathbb{C}} \backslash \mathcal{J}(f)$, then by equation (4.1.2) there exists some neighbourhood $U_{x}$ of $x$ such that $f^{n}$ is well defined for all $n \in \mathbb{N}$ and $\operatorname{card}\left(\hat{\mathbb{C}} \backslash \mathcal{O}_{f}^{+}\left(U_{x}\right)\right)=\infty$. Now by 
Montel's Theorem, $\left\{f^{n}: n \in \mathbb{N}\right\}$ forms a normal family on $U_{x}$, thus $x \in \mathcal{F}(f)$.

Conversely, let $x \in \mathcal{F}(f)$. Then there exists an open neighbourhood $V_{x}$ of $x$ such that $f^{n}$ is well defined for all $n \in \mathbb{N}$ and $\left\{f^{n}: n \in \mathbb{N}\right\}$ forms a normal family on $V_{x}$. Since $\mathcal{F}(f)$ is completely invariant, then $\mathcal{O}_{f}^{+}\left(V_{x}\right) \subset \mathcal{F}(f)$ and therefore $\mathcal{J}_{\text {mero }}(f) \subset \hat{\mathbb{C}} \backslash \mathcal{O}_{f}^{+}\left(V_{x}\right)$. Finally, as $\mathcal{J}_{\text {mero }}(f)$ is infinite then card $\left(\hat{\mathbb{C}} \backslash \mathcal{O}_{f}^{+}\left(V_{x}\right)\right)=\infty$. Hence $x \in \hat{\mathbb{C}} \backslash \mathcal{J}(f)$ and $\mathcal{J}_{\text {mero }}(f)=\mathcal{J}(f)$ as required.

More crucially, it turns out that $\mathcal{J}(f)$ is non-empty and has analogous properties to those of Theorem 1.1.3(i)-(vi). This results in the following theorem, which will form the main focus for the rest of this chapter.

Theorem 4.1.3. Let $f: \mathbb{R}^{d} \rightarrow \hat{\mathbb{R}}^{d}$ be a quasimeromorphic mapping of transcendental type with at least one pole. Then the following hold.

(i) $\mathcal{J}(f) \neq \varnothing$. In fact, $\operatorname{card}(\mathcal{J}(f))=\infty$.

(ii) $\mathcal{J}(f)$ is closed and does not contain any isolated points.

(iii) $x \in \mathcal{J}(f) \backslash\{\infty\}$ if and only if $f(x) \in \mathcal{J}(f)$. In particular, $\mathcal{J}(f) \backslash \mathcal{O}_{f}^{-}(\infty)$ is completely invariant.

(iv) $\mathcal{J}(f) \subset \overline{\mathcal{O}_{f}^{-}(x)}$ for every $x \in \hat{\mathbb{R}}^{d} \backslash E(f)$.

(v) $\mathcal{J}(f)=\overline{\mathcal{O}_{f}^{-}(x)}$ for every $x \in \mathcal{J}(f) \backslash E(f)$.

(vi) If $U \subset \hat{\mathbb{R}}^{d}$ is an open set such that $U \cap \mathcal{J}(f) \neq \varnothing$, then $\hat{\mathbb{R}}^{d} \backslash E(f) \subset \mathcal{O}_{f}^{+}(U)$.

(vii) For each $n \in \mathbb{N}$, if $2 \leq \operatorname{card}\left(\mathcal{O}_{f}^{-}(\infty)\right)<q$, then

$$
\begin{aligned}
& \mathcal{J}(f)=\{\left\{x \in \hat{\mathbb{R}}^{d} \backslash \overline{\mathcal{O}_{f}^{-}(\infty)}: \operatorname{card}\left(\hat{\mathbb{R}}^{d} \backslash \mathcal{O}_{f^{n}}^{+}\left(U_{x}\right)\right)<\infty\right. \text { for all } \\
&\text { neighbourhoods } \left.U_{x} \subset \hat{\mathbb{R}}^{d} \backslash \overline{\mathcal{O}_{f}^{-}(\infty)} \text { of } x\right\} \cup \overline{\mathcal{O}_{f}^{-}(\infty)} . \\
& \text { If } \operatorname{card}\left(\mathcal{O}_{f}^{-}(\infty)\right) \geq q \text {, then } \mathcal{J}(f)=\overline{\mathcal{O}_{f}^{-}(\infty)}=\overline{\mathcal{O}_{f^{n}}^{-}(\infty)} .
\end{aligned}
$$


When the backward orbit of infinity is finite, then quasimeromorphic mappings of transcendental type with at least one pole are closely related to quasiregular mappings in $S$-punctured space (see Section 4.1 .1 below). These mappings form the quasiregular analogue of holomorphic self-maps of the punctured plane $\mathbb{C}^{*}$ and have been studied by Nicks and Sixsmith in [70]. In particular, it was shown that Conjecture 1.3.6 holds for such mappings; this formed the content of [70, Proposition 3.5]. Motivated by this, it has been possible to show that Conjecture 1.3.6 also holds true in the new setting; this provides support towards the conjecture holding for all non-uniform quasiregular mappings.

Theorem 4.1.4. Let $f: \mathbb{R}^{d} \rightarrow \hat{\mathbb{R}}^{d}$ be a quasimeromorphic mapping of transcendental type with at least one pole. Then $x \in E(f)$ if and only if cap $\left(\overline{\mathcal{O}_{f}^{-}(x)}\right)=0$.

\subsubsection{Quasiregular mappings in $S$-punctured space}

Let $d \geq 2$, let $n \in \mathbb{N}$ be fixed and let $S:=\left\{\infty, s_{1}, s_{2}, \ldots, s_{n}\right\}$ be a finite set of distinct points in $\hat{\mathbb{R}}^{d}$. Then a quasiregular mapping $g: \hat{\mathbb{R}}^{d} \backslash S \rightarrow \hat{\mathbb{R}}^{d} \backslash S$ is said to be of $S$-transcendental type if $S$ coincides with the set of essential singularities of $g$. We note that it is important to have $n \geq 1$, so $\operatorname{card}(S) \geq 2$, to distinguish these mappings from quasiregular mappings of transcendental type. The Julia set $\mathcal{J}_{S}(g)$ is then defined as

$$
\begin{aligned}
\mathcal{J}_{S}(g)=\{ & x \in \hat{\mathbb{R}}^{d} \backslash S: \operatorname{card}\left(\hat{\mathbb{R}}^{d} \backslash \mathcal{O}_{g}^{+}\left(U_{x}\right)\right)<\infty \text { for all } \\
& \text { neighbourhoods } \left.U_{x} \subset \hat{\mathbb{R}}^{d} \backslash S \text { of } x\right\} .
\end{aligned}
$$

Recall from Section 1.3 the degree of a quasiregular mapping on $\mathbb{R}^{d}$. It was noted by Nicks and Sixsmith [70] that quasiregular mappings in $S$-punctured space can be constructed by composing together a quasiregular mapping of transcendental type $f: \mathbb{R}^{d} \rightarrow \mathbb{R}^{d} \backslash\left\{s_{1}, s_{2}, \ldots, s_{n}\right\}$, which exists by Drasin and Pankka [27], 
and a quasiregular map of polynomial type $g: \hat{\mathbb{R}}^{d} \rightarrow \hat{\mathbb{R}}^{d}$ of degree $p+1$, where $g^{-1}(\infty)=\left\{\infty, s_{1}, s_{2}, \ldots, s_{n}\right\}$.

Another way to construct a quasiregular mapping in $S$-punctured space is through iterating a quasimeromorphic mapping of transcendental type where the backward orbit of infinity is finite, but non-empty. Indeed, suppose that $f: \mathbb{R}^{d} \rightarrow \hat{\mathbb{R}}^{d}$ is a $K$-quasimeromorphic mapping of transcendental type with $2 \leq \operatorname{card}\left(\mathcal{O}_{f}^{-}(\infty)\right)<q$ and consider the iterate $f^{q}$ restricted to the domain $\mathbb{R}^{d} \backslash \mathcal{O}_{f}^{-}(\infty)$. As $\mathcal{O}_{f}^{-}(\infty)$ is backward invariant, then every point of $\mathcal{O}_{f}^{-}(\infty)$ will be an omitted value of $f^{q}$. Further as infinity is an essential singularity of $f$, then all points in $\mathcal{O}_{f}^{-}(\infty)$ will be essential singularities of $f^{q}$. This means that $f^{n}: \mathbb{R}^{d} \backslash \mathcal{O}_{f}^{-}(\infty) \rightarrow \mathbb{R}^{d} \backslash \mathcal{O}_{f}^{-}(\infty)$ will be a quasiregular mapping of $S$-transcendental type for all $n \geq q$, where $S=\mathbb{R}^{d} \backslash \mathcal{O}_{f}^{-}(\infty)$

Example 4.1.5. Let $f: \mathbb{R}^{3} \rightarrow \mathbb{R}^{3}$ be a quasimeromorphic mapping of transcendental type satisfying equation (2.1.2) from Corollary 2.1.3. It follows that $f^{2}: \mathbb{R}^{3} \backslash\{0\} \rightarrow \mathbb{R}^{3} \backslash\{0\}$ is a quasiregular mapping of $S$-transcendental type, where $S=\{0, \infty\}$.

We now summarise some of the results shown for $\mathcal{J}_{S}(g)$ in [70] in the following theorem. Here, any closure is taken with respect to $\hat{\mathbb{R}}^{d} \backslash S$, unless stated otherwise. We note that the proof of these results in [70] hold after using Theorem 3.1.2 from Chapter 3 to correct an omission there.

Theorem 4.1.6. Let $S \subset \hat{\mathbb{R}}^{d}$ be a finite set with $\infty \in S$ and $\operatorname{card}(S) \geq 2$, and suppose that $g: \hat{\mathbb{R}}^{d} \backslash S \rightarrow \hat{\mathbb{R}}^{d} \backslash S$ is a quasiregular map of $S$-transcendental type. Then the following hold:

(i) $\mathcal{J}_{S}(g)$ is closed, infinite and does not contain any isolated points;

(ii) $x \in \mathcal{J}_{S}(g)$ if and only if $g(x) \in \mathcal{J}_{S}(g)$;

(iii) for all $x \in \mathbb{R}^{d} \backslash E(g)$, we have $\mathcal{J}_{S}(g) \subset \overline{\mathcal{O}_{g}^{-}(x)}$; 
(iv) for all $x \in \mathcal{J}_{S}(g) \backslash E(g)$, we have $\mathcal{J}_{S}(g)=\overline{\mathcal{O}_{g}^{-}(x)}$;

(v) $\mathcal{J}_{S}(g)=\mathcal{J}_{S}\left(g^{k}\right)$ for each $k \in \mathbb{N}$; and

(vi) the closure of all components of $\mathcal{J}_{S}(g)$ with respect to $\hat{\mathbb{R}}^{d}$ meet $S$.

\subsection{Proof of Theorem 4.1.3 when $\mathcal{O}_{f}^{-}(\infty)$ is finite}

Suppose that $f: \mathbb{R}^{d} \rightarrow \hat{\mathbb{R}}^{d}$ is a $K$-quasimeromorphic mapping of transcendental type with $2 \leq \operatorname{card}\left(\mathcal{O}_{f}^{-}(\infty)\right)<q$, where $q$ is Rickman's constant from Theorem 1.2.6. As noted above, the iterates $f^{n}: \mathbb{R}^{d} \backslash S \rightarrow \mathbb{R}^{d} \backslash S$ are all quasiregular mappings of $S$-transcendental type for all $n \geq q$, where $S=\mathcal{O}_{f}^{-}(\infty)$. From this, we may appeal to the Fatou-Julia theory for quasiregular mappings on $S$-punctured space; this provides an approach to proving Theorem 4.1.3.

Firstly, we shall prove a result concerning the relationship between $\mathcal{J}(f)$ and $\mathcal{J}_{S}\left(f^{n}\right)$ for $n \geq q$. For this, we require a few observations. Indeed, note that by applying Theorem 4.1.6(v) twice, then for all $n \geq q$,

$$
\mathcal{J}_{S}\left(f^{n}\right)=\mathcal{J}_{S}\left(f^{n q}\right)=\mathcal{J}_{S}\left(f^{q}\right) .
$$

In particular, we have that $\mathcal{J}_{S}\left(f^{q}\right)=\mathcal{J}_{S}\left(f^{q+1}\right)$. It now follows from Theorem 4.1.6(ii) that

$$
\begin{aligned}
f(x) \in \mathcal{J}_{S}\left(f^{q}\right) & \Longleftrightarrow f^{q+1}(x) \in \mathcal{J}_{S}\left(f^{q}\right) \\
& \Longleftrightarrow f^{q+1}(x) \in \mathcal{J}_{S}\left(f^{q+1}\right) \\
& \Longleftrightarrow x \in \mathcal{J}_{S}\left(f^{q+1}\right) \\
& \Longleftrightarrow x \in \mathcal{J}_{S}\left(f^{q}\right) .
\end{aligned}
$$


Theorem 4.2.1. Let $f: \mathbb{R}^{d} \rightarrow \hat{\mathbb{R}}^{d}$ be a quasimeromorphic mapping of transcendental type with at least one pole and let q be Rickman's constant. Suppose that $S=\mathcal{O}_{f}^{-}(\infty)$ is finite. Then for all $n \geq q$,

$$
\mathcal{J}(f)=\mathcal{J}_{S}\left(f^{n}\right) \cup S
$$

Proof. Using equation (4.2.1) and Definition 4.1.1, it will suffice to prove that $\mathcal{J}(f) \backslash \mathcal{O}_{f}^{-}(\infty)=\mathcal{J}_{S}\left(f^{q}\right)$. Indeed, firstly note that the reverse inclusion is clear. This is because for any open neighbourhood $U_{x} \subset \hat{\mathbb{R}}^{d} \backslash \mathcal{O}_{f}^{-}(\infty)$ of a point $x \in \mathcal{J}_{S}\left(f^{q}\right)$, we have

$$
\hat{\mathbb{R}}^{d} \backslash \mathcal{O}_{f}^{+}\left(U_{x}\right) \subset \hat{\mathbb{R}}^{d} \backslash \mathcal{O}_{f^{q}}^{+}\left(U_{x}\right)
$$

This means that if $\hat{\mathbb{R}}^{d} \backslash \mathcal{O}_{f^{q}}^{+}\left(U_{x}\right)$ is finite, then $\hat{\mathbb{R}}^{d} \backslash \mathcal{O}_{f}^{+}\left(U_{x}\right)$ is finite as well.

For the other direction, let $x \in \hat{\mathbb{R}}^{d} \backslash \mathcal{O}_{f}^{-}(\infty)$ be such that for any open neighbourhood $V_{x} \subset \hat{\mathbb{R}}^{d} \backslash \mathcal{O}_{f}^{-}(\infty)$ of $x$, then $\operatorname{card}\left(\hat{\mathbb{R}}^{d} \backslash \mathcal{O}_{f}^{+}\left(V_{x}\right)\right)$ is finite. Since $\mathcal{J}_{S}\left(f^{q}\right)$ is infinite from Theorem 4.1.6(i), then we must have that $\mathcal{O}_{f}^{+}\left(V_{x}\right) \cap \mathcal{J}_{S}\left(f^{q}\right) \neq \varnothing$. It follows from equation (4.2.2) that $V_{x} \cap \mathcal{J}_{S}\left(f^{q}\right) \neq \varnothing$. Finally as $\mathcal{J}_{S}\left(f^{q}\right)$ is closed in $\hat{\mathbb{R}}^{d} \backslash \mathcal{O}_{f}^{-}(\infty)$ and $V_{x}$ was an arbitrary open neighbourhood of $x$, then $x \in \mathcal{J}_{S}\left(f^{q}\right)$, concluding the proof of Theorem 4.2.1.

With Theorem 4.2.1 established, we can turn to the proof of Theorem 4.1.3. Indeed, firstly observe that Theorem 4.1.6 states that many properties of the classical Julia set hold for $\mathcal{J}_{S}\left(f^{n}\right)$ with $n \geq q$. In particular, Theorem 4.1.6(vi) gives us that the closure of every component $Y \subset \hat{\mathbb{R}}^{d} \backslash \mathcal{O}_{f}^{-}(\infty)$ of $\mathcal{J}_{S}\left(f^{q}\right)$ meets $\mathcal{O}_{f}^{-}(\infty)$. This means that with trivial extensions to the arguments in [70], to encompass the case when $x \in \mathcal{O}_{f}^{-}(\infty)$, we find that the properties listed in Theorem 4.1.6 also hold for $\mathcal{J}_{S}\left(f^{n}\right) \cup S$ for $n \geq q$, where $S=\mathcal{O}_{f}^{-}(\infty)$. Therefore Theorem 4.1.3(i)-(v) follows immediately from Theorem 4.1.6.

Next Theorem 4.1.3(vi) follows from Theorem 4.1.3(iv). This is because for any 
$x \in \mathbb{R}^{d} \backslash E(f)$, any open set $U \subset \mathbb{R}^{d}$ intersecting $\mathcal{J}(f)$ must also non-trivially intersect $\mathcal{O}_{f}^{-}(x)$.

Finally to prove Theorem 4.1.3(vii), observe that the reverse inclusion is clear. This is because for any set $U \subset \hat{\mathbb{R}}^{d} \backslash S$ such that $\operatorname{card}\left(\hat{\mathbb{R}}^{d} \backslash \mathcal{O}_{f^{n}}^{+}\left(U_{x}\right)\right)$ is finite, then $\operatorname{card}\left(\hat{\mathbb{R}}^{d} \backslash \mathcal{O}_{f}^{+}\left(U_{x}\right)\right)$ is finite.

For the other direction, for any given $n \in \mathbb{N}$ we have $\mathcal{J}(f) \backslash S=\mathcal{J}_{S}\left(f^{n q}\right)$ by Theorem 4.2.1. Now let $x \in \mathcal{J}(f) \backslash S$, so card $\left(\hat{\mathbb{R}}^{d} \backslash \mathcal{O}_{f^{n q}}^{+}\left(V_{x}\right)\right)<\infty$ for any neighbourhood $V_{x} \subset \hat{\mathbb{R}}^{d} \backslash S$ of $x$. This implies that card $\left(\hat{\mathbb{R}}^{d} \backslash \mathcal{O}_{f^{n}}^{+}\left(V_{x}\right)\right)<\infty$. Since $V_{x}$ was an arbitrary neighbourhood of $x$, then the result follows. This completes the proof of Theorem 4.1.3 when $\mathcal{O}_{f}^{-}(\infty)$ is finite.

\subsection{Proof of Theorem 4.1.3 when $\mathcal{O}_{f}^{-}(\infty)$ is infinite}

Throughout this section, we shall assume that $f: \mathbb{R}^{d} \rightarrow \hat{\mathbb{R}}^{d}$ is a quasimeromorphic mapping of transcendental type and $\operatorname{card}\left(\mathcal{O}_{f}^{-}(\infty)\right)=\infty$. We recall by an earlier remark that $\mathcal{J}(f)=\overline{\mathcal{O}_{f}^{-}(\infty)}$ in this case; we shall use this to prove Theorem 4.1.3. In what follows, for each $N \in \mathbb{N}$ we shall denote $P(N)=\bigcup_{n=0}^{N-1} f^{-n}(\infty)$, so that $f^{N}: \mathbb{R}^{d} \backslash P(N) \rightarrow \hat{\mathbb{R}}^{d}$ is also a quasimeromorphic mapping of transcendental type. Further, we recall that $f^{0}=\mathrm{id}: \hat{\mathbb{R}}^{d} \rightarrow \hat{\mathbb{R}}^{d}$.

Firstly observe that by the definition, Theorem 4.1.3(i) is clearly satisfied and $\mathcal{J}(f)$ is closed. Next, Theorem 4.1.3(ii) will follow from the following lemma.

Lemma 4.3.1. $\mathcal{J}(f)$ does not contain any isolated points.

Proof. To prove this, it shall suffice to show that for each $x \in \mathcal{O}_{f}^{-}(\infty)$, every open neighbourhood $U_{x}$ of $x$ is such that $\left(U_{x} \backslash\{x\}\right) \cap \mathcal{O}_{f}^{-}(\infty) \neq \varnothing$. Indeed, fix some arbitrary $x \in \mathcal{O}_{f}^{-}(\infty)$ and open neighbourhood $U_{x}$ of $x$, so there exists some $N \geq 0$ 
such that $f^{N}(x)=\infty$. If $N \in \mathbb{N}$, then $f^{N}: \mathbb{R}^{d} \backslash P(N) \rightarrow \hat{\mathbb{R}}^{d}$ is a quasimeromorphic mapping, so in particular continuous, discrete and open. Then there exists some open neighbourhood $V_{x} \subset U_{x}$ containing $x$ such that $f^{N}$ is quasimeromorphic on $V_{x}, V_{x} \cap f^{-N}(\infty)=\{x\}$ and $f^{N}\left(V_{x}\right)$ is an open set around infinity. If $N=0$, then $f^{0}=$ id and $V_{x}=V_{\infty}$ is an open neighbourhood of infinity.

Since $\operatorname{card}\left(\mathcal{O}_{f}^{-}(\infty)\right)=\infty$, then it follows from Theorem 1.2.6 that there exists some $s \in\left(\mathcal{O}_{f}^{-}(\infty) \backslash\{\infty\}\right) \cap f^{N}\left(V_{x}\right)$. This implies that there exists some $v_{s} \in V_{x}$ such that $f^{N}\left(v_{s}\right)=s$, whence $v_{s} \in \mathcal{O}_{f}^{-}(\infty)$. It remains to note that $v_{s} \neq x$ since $f^{N}\left(v_{s}\right) \neq \infty$. Thus $\left(V_{x} \backslash\{x\}\right) \cap \mathcal{O}_{f}^{-}(\infty) \neq \varnothing$, and so $\left(U_{x} \backslash\{x\}\right) \cap \mathcal{O}_{f}^{-}(\infty) \neq \varnothing$ as required.

For Theorem 4.1.3(iii), first let $x \in \mathcal{J}(f) \backslash\{\infty\}$. If $x \in \mathcal{O}_{f}^{-}(\infty) \backslash\{\infty\}$, then there exists some $N \in \mathbb{N}$ such that $f^{N}(x)=\infty$. Now clearly $f(x)$ is defined with $f^{N-1}(f(x))=\infty$, thus $f(x) \in \mathcal{J}(f)$. If $x \in \overline{\mathcal{O}_{f}^{-}(\infty)} \backslash \mathcal{O}_{f}^{-}(\infty)$, then there exist $x_{n} \in \mathcal{O}_{f}^{-}(\infty) \backslash\{\infty\}$ such that $x_{n} \rightarrow x$ as $n \rightarrow \infty$. It then follows that $f\left(x_{n}\right)$ exists for each $n \in \mathbb{N}$. As $f$ is continuous, then $f\left(x_{n}\right) \rightarrow f(x)$ as $n \rightarrow \infty$. Therefore as $\mathcal{J}(f)$ is closed, we conclude that $f(x) \in \mathcal{J}(f)$. In particular since $x \notin \mathcal{O}_{f}^{-}(\infty)$, then $f(x) \notin \mathcal{O}_{f}^{-}(\infty)$ either, so $f(x) \in \mathcal{J}(f) \backslash \mathcal{O}_{f}^{-}(\infty)$.

For the other direction, let $f(x) \in \mathcal{J}(f)$ for some $x \in \mathbb{R}^{d}$. If $f(x) \in \mathcal{O}_{f}^{-}(\infty)$, then $x \in \mathcal{O}_{f}^{-}(\infty) \backslash\{\infty\}$ by definition. So suppose that $f(x) \in \overline{\mathcal{O}_{f}^{-}(\infty)} \backslash \mathcal{O}_{f}^{-}(\infty)$ and let $U$ be a neighbourhood of $x$. Since $f(x)$ is defined and $f$ is an open mapping, then there exists some neighbourhood $V$ of $f(x)$ such that $f(U) \supset V$. As $f(x)$ is a limit point of $\mathcal{O}_{f}^{-}(\infty)$, there exists some $y_{n} \rightarrow f(x)$ such that $y_{n} \in V \cap \mathcal{O}_{f}^{-}(\infty)$ for all large $n$. This means for all large $n \in \mathbb{N}$ there exists $x_{n} \in U \cap \mathcal{O}_{f}^{-}(\infty)$ with $f\left(x_{n}\right)=y_{n}$. As $U$ was an arbitrary neighbourhood, then we must have $x_{n} \rightarrow x$ as $n \rightarrow \infty$, thus $x \in \overline{\mathcal{O}_{f}^{-}(\infty)} \backslash\{\infty\}=\mathcal{J}(f) \backslash\{\infty\}$.

Next, we shall prove Theorem 4.1.3(iv) in the following lemma. 
Lemma 4.3.2. For all $x \in \hat{\mathbb{R}}^{d} \backslash E(f), \mathcal{J}(f) \subset \overline{\mathcal{O}_{f}^{-}(x)}$.

Proof. Let $x \in \hat{\mathbb{R}}^{d} \backslash E(f)$. Note that the result is trivial if $x=\infty$, so assume that $x \neq \infty$. Now since $x \notin E(f)$, then $\operatorname{card}\left(\mathcal{O}_{f}^{-}(x)\right)=\infty$. As $E(f)$ is a finite set, then by Theorem 1.2.6 there exists $w_{n} \rightarrow \infty$ such that for each $n \in \mathbb{N}, w_{n} \in \mathcal{O}_{f}^{-}(x)$ and $\operatorname{card}\left(\mathcal{O}_{f}^{-}\left(w_{n}\right)\right)=\infty$

Let $y \in \mathcal{O}_{f}^{-}(\infty)$ and let $U_{y}$ be an open neighbourhood of $y$. Then there exists some $k \geq 0$ such that $f^{k}(y)=\infty$. It follows that there exists some open neighbourhood $V_{y} \subset U_{y}$ containing $y$ such that $f^{k}$ is either quasimeromorphic or the identity mapping on $V_{y}$. Now as $f^{k}$ is open, then $f^{k}\left(V_{x}\right)$ will be an open set around infinity. Since $w_{n} \rightarrow \infty$, then there exists some $N \in \mathbb{N}$ such that $w_{n} \in f^{k}\left(V_{x}\right)$ whenever $n \geq N$. In particular, there exists some $y_{N} \in V_{y} \subset U_{y}$ such that $f^{k}\left(y_{N}\right)=w_{N}$. It follows that $y_{N} \in \mathcal{O}_{f}^{-}\left(w_{N}\right) \subset \mathcal{O}_{f}^{-}(x)$, hence $U_{y} \cap \mathcal{O}_{f}^{-}(x) \neq \varnothing$. As $U_{y}$ was an arbitrary neighbourhood of $y$, then $y \in \overline{\mathcal{O}_{f}^{-}(x)}$ as required.

It is easy to see that Theorem 4.1.3(v) follows immediately from Theorem 4.1.3(iv); if $x \in \mathcal{J}(f) \backslash E(f)$, then $\mathcal{J}(f) \subset \overline{\mathcal{O}_{f}^{-}(x)}$ follows from Lemma 4.3.2, while the other direction follows from Theorem 4.1.3(iii) and the fact that $\mathcal{J}(f)$ is closed. Further, Theorem 4.1.3(vi) also follows immediately from Theorem 4.1.3(iv). This is because for every $x \in \hat{\mathbb{R}}^{d} \backslash E(f)$, any open set $U \subset \hat{\mathbb{R}}^{d}$ intersecting $\mathcal{J}(f)$ must also nontrivially intersect $\mathcal{O}_{f}^{-}(x)$.

Finally we shall prove Theorem 4.1.3(vii) in the following lemma.

Lemma 4.3.3. For each $n \in \mathbb{N}, \mathcal{J}(f)=\overline{\mathcal{O}_{f}^{-}(\infty)}=\overline{\mathcal{O}_{f^{n}}^{-}(\infty)}$.

Proof. First note that $\overline{\mathcal{O}_{f^{n}}^{-}(\infty)} \subset \overline{\mathcal{O}_{f}^{-}(\infty)}$ is obvious by definition. For the other inclusion, let $x \in \mathcal{O}_{f}^{-}(\infty)$ and note that from Theorem 1.2.6, $\operatorname{card}\left(\mathcal{O}_{f}^{-}(\infty)\right)=\infty$ implies that $f^{-N}(\infty)$ is infinite for all $N \geq q$.

Let $U_{x}$ be an open neighbourhood of $x$, so $U_{x} \cap \mathcal{O}_{f}^{-}(\infty) \neq \varnothing$. Let $y \in U_{x} \cap \mathcal{O}_{f}^{-}(\infty)$, so 
there exists some $k \geq 0$ such that $f^{k}(y)=\infty$. If $k \in \mathbb{N}$, then $f^{k}: \mathbb{R}^{d} \backslash P(k) \rightarrow \hat{\mathbb{R}}^{d}$ is a quasimeromorphic mapping. Then there exists some open neighbourhood $V_{y} \subset U_{x}$ containing $y$ such that $f^{k}$ is quasimeromorphic on $V_{y}$ and $f^{k}\left(V_{y}\right)$ is an open set around infinity. If $k=0$, then $f^{0}=\mathrm{id}$ and $V_{y}=V_{\infty}$ is an open neighbourhood of infinity.

Let $a \in \mathbb{N}$ be sufficiently large such that $a n-k>q$, so $f^{-(a n-k)}(\infty)$ is infinite. It follows that there exists some $w \in f^{k}\left(V_{y}\right) \cap f^{-(a n-k)}(\infty)$. Now there exists $u \in V_{y}$ such that $f^{k}(u)=w$, hence

$$
f^{a n}(u)=f^{a n-k+k}(u)=f^{a n-k}(w)=\infty,
$$

and so $u \in \mathcal{O}_{f^{n}}(\infty)$. As $V_{y} \subset U_{x}$ and $U_{x}$ was arbitrary, then $x \in \overline{\mathcal{O}_{f^{n}}^{-}(\infty)}$ as required. This completes the proof of Theorem 4.1.3.

\subsection{Proof of Theorem 4.1.4}

Suppose that $f: \mathbb{R}^{d} \rightarrow \hat{\mathbb{R}}^{d}$ is a $K$-quasimeromorphic mapping of transcendental type with at least one pole. By considering a large iterate of $f$ and [70, Proposition 3.5], which proved Theorem 4.1.4 for quasiregular mappings in $S$-punctured space, the case of Theorem 4.1.4 when $\mathcal{O}_{f}^{-}(\infty)$ is finite has already been established. Hence within this section, we shall only consider the case when $\operatorname{card}\left(\mathcal{O}_{f}^{-}(\infty)\right)=\infty$. Let $x \in \hat{\mathbb{R}}^{d}$ and note that it is sufficient to show that $x \in \hat{\mathbb{R}}^{d} \backslash E(f)$ implies $\operatorname{cap}\left(\overline{\mathcal{O}_{f}^{-}(x)}\right)>0$, as the reverse implication is obvious. To this end, we shall state a sufficient condition which consists of Lemma 2.9, Lemma 2.10, and Lemma 2.11 from [17]. These lemmas together essentially state that if every point $y$ in a compact set $X$ has sufficiently many disjoint preimages in $X$, then $\operatorname{cap}\left(\overline{\mathcal{O}_{f}^{-}(y)}\right)>0$.

Lemma 4.4.1. Let $\delta>0$, let $X \subset \mathbb{R}^{d}$ be a compact set and let $f: X \rightarrow \hat{\mathbb{R}}^{d}$ be a $K$-quasimeromorphic mapping. Suppose that there exists $m \in \mathbb{N}$ such that every 
$y \in X$ has $m$ distinct points $x_{1}, x_{2}, \ldots, x_{m} \in f^{-1}(y)$, with $\left|x_{i}-x_{j}\right| \geq \delta$ for all $i \neq j$. If $m>K$, then $\operatorname{cap}\left(\overline{\mathcal{O}_{f}^{-}(y)}\right)>0$ for all $y \in X$.

It should be noted that [17] only refers to quasiregular mappings, although the above statement follows as we may remove small neighbourhoods of any poles from $X$.

We shall now prove a covering result for the neighbourhoods of poles. For notation, recall that $x$ is an $m$-prepole of $f$ if $f^{m}(x)=\infty$ for some $m \in \mathbb{N}$, where a 1-prepole is precisely the same as a pole. Observe that if $\operatorname{card}\left(\mathcal{O}_{f}^{-}(\infty)\right)=\infty$, then there exist some point $y \in \mathcal{O}_{f}^{-}(\infty)$ and $N \in \mathbb{N}$ such that $y$ is an $N$-prepole of $f$ and $\operatorname{card}\left(f^{-1}(y)\right)=\infty$.

Lemma 4.4.2. Let $f: \mathbb{R}^{d} \rightarrow \hat{\mathbb{R}}^{d}$ be a quasimeromorphic mapping of transcendental type. Suppose that there exists a bounded open neighbourhood $U \subset \mathbb{R}^{d}$ of an $N$ prepole of $f$ such that $f^{N}$ is quasimeromorphic on $U$ and $f^{-1}(u)$ is infinite for all $u \in \bar{U}$. Then given any $r>0$, there exists a bounded open set $E_{U} \subset A(r, \infty)$ such that $f^{N}(U) \supset \overline{E_{U}}$ and $f\left(E_{U}\right) \supset \bar{U}$.

Proof. Since $f^{N}$ is quasimeromorphic on $U$ and $U$ is an open set containing an $N$-prepole of $f$, then $f^{N}(U)$ is an open set covering infinity. Now by assuming without loss of generality that $r>0$ is sufficiently large, then $\overline{A(r, \infty)} \subset f^{N}(U)$. Let $u \in \bar{U}$. Since $f^{-1}(u)$ is infinite and $f$ is a discrete mapping, there exists $x(u) \in A(r+1, \infty)$ such that $f(x(u))=u$. As $f$ is open, then $B(x(u), 1) \subset A(r, \infty)$ and $f(B(x(u), 1)) \supset B(u, \delta(u))$ for some $\delta(u)>0$. Thus we can construct an open cover

$$
\bigcup_{u \in \bar{U}} f(B(x(u), 1)) \supset \bigcup_{u \in \bar{U}} B(u, \delta(u)) \supset \bar{U}
$$

As $\bar{U}$ is bounded, then it is compact. Hence there exists some $n \in \mathbb{N}$ and $u_{i} \in \bar{U}$, 
$i=1,2, \ldots, n$, such that

$$
\bigcup_{i=1}^{n} f\left(B\left(x\left(u_{i}\right), 1\right)\right) \supset \bar{U}
$$

Now define

$$
E_{U}:=\bigcup_{i=1}^{n} B\left(x\left(u_{i}\right), 1\right)
$$

Observing that $B\left(x\left(u_{i}\right), 1\right) \subset A(r, \infty)$ for each $i=1,2, \ldots, n$, it follows that $\overline{E_{U}} \subset \overline{A(r, \infty)} \subset f^{N}(U)$. Furthermore, as each $B\left(x\left(u_{i}\right), 1\right)$ is bounded, then $E_{U}$ is bounded. Hence from equations (4.4.1) and (4.4.2), $E_{U}$ is the bounded set as required, completing the proof of Lemma 4.4.2.

Proof of Theorem 4.1.4. Firstly, we claim that for some $N \in \mathbb{N}$, there exists an $N$-prepole $y$ of $f$ and a bounded neighbourhood $U_{y}$ of $y$ such that cap $\left(\overline{\mathcal{O}_{f}^{-}(w)}\right)>0$ for all $w \in \overline{U_{y}}$. Indeed as $\operatorname{card}\left(\mathcal{O}_{f}^{-}(\infty)\right)=\infty$, then using Theorem 1.2.6 and the fact that $f^{n}$ is quasimeromorphic on $\mathbb{R}^{d} \backslash P(n)$ for all $n \in \mathbb{N}$, then there must exist some $N \in \mathbb{N}$, some $y \in \mathcal{O}_{f}^{-}(\infty)$ and some bounded neighbourhood $U_{y}$ of $y$ such that $y$ is an $N$-prepole of $f, \operatorname{card}\left(f^{-1}(u)\right)=\infty$ for all $u \in \overline{U_{y}}, f^{N}$ is quasimeromorphic on $U_{y}$ and $U_{y} \cap f^{-N}(\infty)=\{y\}$.

We shall inductively define a strictly increasing real sequence $r_{n} \rightarrow \infty$ and a sequence of bounded open sets $E_{n}$ as follows. Fix some $r_{1}>0$ and let $E_{1}$ be the bounded open set found by applying Lemma 4.4 .2 with $r_{1}$ and $U_{y}$. Now for each $n \in \mathbb{N}$, inductively define $r_{n+1}=\sup \left\{|x|: x \in E_{n}\right\}+1$ and set $E_{n+1}$ as the bounded open set found by applying Lemma 4.4 .2 with $r_{n+1}$ and $U_{y}$. This gives a collection of bounded open sets $\left\{E_{n}: n \in \mathbb{N}\right\}$ with pairwise disjoint closures such that for all $n \in \mathbb{N}$,

$$
f^{N}\left(U_{y}\right) \supset \overline{E_{n}} \text { and } f\left(E_{n}\right) \supset \overline{U_{y}} \text {. }
$$

Since these sets have pairwise disjoint closures, then there must exist pairwise disjoint closed sets $V_{n} \subset U_{y}$ such that for each $n \in \mathbb{N}, V_{n}$ contains no $N$-prepoles of 
$f$ and $f^{N}\left(V_{n}\right) \supset \overline{E_{n}}$. In particular, this means that $f^{N+1}\left(V_{n}\right) \supset \overline{U_{y}}$ for each $n \in \mathbb{N}$. For any $m \in \mathbb{N}$, observe that $f^{N+1}$ is a $K^{N+1}$-quasimeromorphic mapping defined on the compact set $\bigcup_{i=1}^{m} V_{i}$. By applying Lemma 4.4.1 to $f^{N+1}$ with $m>K^{N+1}$, then for all $w \in \overline{U_{y}}$, we have that $\operatorname{cap}\left(\overline{\mathcal{O}_{f^{N+1}}^{-}(w)}\right)>0$. Since $\overline{\mathcal{O}_{f^{N+1}}^{-}(w)} \subset \overline{\mathcal{O}_{f}^{-}(w)}$ by definition, then $\operatorname{cap}\left(\overline{\mathcal{O}_{f}^{-}(w)}\right)>0$ for all $w \in \overline{U_{y}}$, proving the claim.

Finally, take some $x \in \hat{\mathbb{R}}^{d} \backslash E(f)$ so $\operatorname{card}\left(\mathcal{O}_{f}^{-}(x)\right)=\infty$. Then by Theorem 1.2.6, there exists some $\alpha \in \mathcal{O}_{f}^{-}(x) \cap f^{N}\left(U_{y}\right)$, where $y$ and $U_{y}$ are as above. In particular, this means that there exists some $u_{\alpha} \in U_{y}$ such that $f^{N}\left(u_{\alpha}\right)=\alpha$. Hence $u_{\alpha} \in \mathcal{O}_{f}^{-}(x)$. As $u_{\alpha} \in \overline{U_{y}}$, then $\operatorname{cap}\left(\overline{\mathcal{O}_{f}^{-}\left(u_{\alpha}\right)}\right)>0$. Therefore we have that $\operatorname{cap}\left(\overline{\mathcal{O}_{f}^{-}(x)}\right)>0$ as required. 


\section{On the existence of slow escaping and non-escaping points}

The focus of this chapter is to use the main results of Chapter 3 and Chapter 4 to show that for any quasimeromorphic mapping with an essential singularity at infinity, there exist points in the Julia set whose iterates tend to infinity arbitrarily slowly. This extends a result by Nicks for quasiregular mappings, and Rippon and Stallard for transcendental meromorphic functions on the complex plane. We further establish basic relationships between the Julia set and the boundaries of the escaping set, the bounded orbit set and the bungee set in the new setting, showing that they are analogous to those for quasiregular mappings.

Much of the content forming this chapter can be found in [108].

\subsection{Introduction}

Let $f: \mathbb{R}^{d} \rightarrow \hat{\mathbb{R}}^{d}$ be a quasimeromorphic mapping of transcendental type. Then by recalling Section 1.4, define the escaping set as

$$
I(f):=\left\{x \in \mathbb{R}^{d}: f^{n}(x) \neq \infty \text { for all } n \in \mathbb{N}, f^{n}(x) \rightarrow \infty \text { as } n \rightarrow \infty\right\},
$$


and define the bounded orbit set and the bungee set respectively as

$$
\begin{aligned}
& B O(f):=\left\{x \in \mathbb{R}^{d}:\left(f^{n}(x)\right)_{n \in \mathbb{N}} \text { is bounded }\right\}, \text { and } \\
& B U(f):=\mathbb{R}^{d} \backslash\left(I(f) \cup B O(f) \cup \mathcal{O}_{f}^{-}(\infty)\right) .
\end{aligned}
$$

For a quasiregular mapping of transcendental type $f$, recall that Nicks in [66] proved Theorem 1.4.6, which states that there always exists a point $x \in I(f) \cap \mathcal{J}(f)$ that escapes to infinity arbitrarily slowly under iteration. The proof of this theorem relies on Bergweiler's growth result, Lemma 3.1.1, together with a holding-up condition to control the rate of escape. Several covering results are then given based on whether the quasiregular mapping has the 'pits effect' or not (see Section 5.2.1), to find sets satisfying the holding-up condition that are sufficiently close to the essential singularity.

As the existence of such slow escaping points has also been shown for transcendental meromorphic functions in the complex plane by Rippon and Stallard [93], then it seems reasonable that slow escaping points also exist for quasimeromorphic mappings of transcendental type with at least one pole. Indeed this is true, and the first part of this chapter will be dedicated to the proof of the following theorem. Here, recall the definition of the Julia set $\mathcal{J}(f)$ from Definition 4.1.1 for a quasimeromorphic mapping $f$ of transcendental type with at least one pole.

Theorem 5.1.1. Let $f: \mathbb{R}^{d} \rightarrow \hat{\mathbb{R}}^{d}$ be a quasimeromorphic map of transcendental type with at least one pole. Then for any positive sequence $a_{n} \rightarrow \infty$, there exists $\zeta \in \mathcal{J}(f)$ and $N \in \mathbb{N}$ such that $\left|f^{n}(\zeta)\right| \rightarrow \infty$ as $n \rightarrow \infty$, while also $\left|f^{n}(\zeta)\right| \leq a_{n}$ whenever $n \geq N$.

The key idea of this proof lies in the observation that if we restrict a quasimeromorphic mapping of transcendental type with finitely many poles to a punctured neighbourhood of infinity, then the resulting mapping will be quasiregular with 
an essential singularity at infinity. Then by suitably replacing Bergweiler's growth result with Theorem 3.1.2, we may adapt the proof structure used by Nicks to get the existence of a slow escaping point for such quasimeromorphic mappings.

When the quasimeromorphic mapping has infinitely many poles, we can adapt a 'pole-hopping' technique similar to that from [14] to achieve the result. This idea is similar to that used by Rippon and Stallard in [93, Section 4] for transcendental meromorphic functions, although the execution is quite different as their method relied on a version of the Ahlfors five islands theorem, which does not extend to the quasimeromorphic setting; see for instance [95]. Instead, the proof given here offers an alternative proof in the meromorphic case which is, in some sense, more elementary.

Next, for a quasiregular mapping of trancendental type $f$, recall the relationships between $\mathcal{J}(f), I(f), B O(f)$ and $B U(f)$ from Theorem 1.4.5. In the new setting, by appealing to the method used in the proof of Theorem 5.1.1 and considering simple modifications of the counterexamples $f_{1}, f_{2}: \mathbb{C} \rightarrow \mathbb{C}$ from Theorem 1.4.5(iii)-(iv), we can show analogous relationships between these sets, without the capacity condition of $\mathcal{J}(f)$. The proof of this theorem and the counterexamples constructed will form the remaining part of this chapter.

Theorem 5.1.2. Let $f: \mathbb{R}^{d} \rightarrow \hat{\mathbb{R}}^{d}$ be a quasimeromorphic map of transcendental type with at least one pole. Then

(i) $I(f) \cap \mathcal{J}(f), B O(f) \cap \mathcal{J}(f)$ and $B U(f) \cap \mathcal{J}(f)$ are infinite, and

(ii) $\mathcal{J}(f) \subset \partial I(f) \cap \partial B O(f) \cap \partial B U(f)$.

As an immediate corollary, by following a similar argument to that given in [74, Proof of Theorem 1.1] it follows that Theorem 1.4.4(iii) holds for all transcendental meromorphic functions $f: \mathbb{C} \rightarrow \hat{\mathbb{C}}$. Furthermore, the proof below showing that $B U(f) \cap \mathcal{J}(f)$ is infinite also holds if $f$ is an entire quasiregular mapping of 
transcendental type. This is because all such points within the proof are found when the mapping is restricted to be a quasiregular mapping of transcendental type on a punctured neighbourhood of infinity. However it still remains open as to whether the capacity condition can be removed entirely from Theorem 1.4.5(ii).

\subsubsection{An orbit lemma for quasimeromorphic mappings}

The following lemma is an extension of [97, Lemma 3.1] to the quasimeromorphic setting. Given a collection of non-empty subsets that form a nested covering sequence, this lemma allows us to find a point in the first set with a specified orbit through all the other sets. Furthermore, this lemma gives sufficient conditions for when we can choose the point to be in the Julia set.

Lemma 5.1.3. Let $f: \mathbb{R}^{d} \rightarrow \hat{\mathbb{R}}^{d}$ be a function. For $n \geq 0$, let $\left(F_{n}\right)$ be a sequence of non-empty bounded sets in $\mathbb{R}^{d},\left(\ell_{n+1}\right)$ be a sequence of natural numbers and $G_{n} \subset F_{n}$ be a sequence of non-empty subsets such that $f^{\ell_{n+1}}$ is continuous on $\overline{G_{n}}$ with

$$
f^{\ell_{n+1}}\left(G_{n}\right) \supset F_{n+1}
$$

For $n \in \mathbb{N}$, set $r_{n}=\sum_{i=1}^{n} \ell_{i}$. Then there exists $\zeta \in \overline{F_{0}}$ such that $f^{r_{n}}(\zeta) \in \overline{F_{n}}$ for each $n \in \mathbb{N}$.

Further, suppose that $f: \mathbb{R}^{d} \rightarrow \hat{\mathbb{R}}^{d}$ is a quasimeromorphic mapping of transcendental type with at least one pole such that for $n \geq 0, f^{\ell_{n+1}}$ is quasimeromorphic on $\overline{G_{n}}$ and equation (5.1.1) holds. If there is a subsequence $\left(F_{n_{k}}\right)$ such that $\overline{F_{n_{k}}} \cap \mathcal{J}(f) \neq \varnothing$ for all $k \in \mathbb{N}$, then $\zeta$ can be chosen to be in $\mathcal{J}(f) \cap \overline{F_{0}}$.

Proof. For all $n \geq 0, f^{\ell_{n+1}}$ is continuous on $\overline{G_{n}}$ and $\overline{G_{n}}$ is compact, so equation (5.1.1) implies that $f^{\ell_{n+1}}\left(\overline{G_{n}}\right) \supset \overline{F_{n+1}}$ for all $n \geq 0$. Now define the sets

$$
T_{N}=\left\{x \in \overline{G_{0}}: f^{r_{n}}(x) \in \overline{G_{n}} \text { for all } n \leq N\right\}
$$


The sets $T_{N}$ are non-empty, compact and form a decreasing nested sequence. Thus $T:=\bigcap_{N=1}^{\infty} T_{N}$ is non-empty and any $\zeta \in T$ is such that $f^{r_{n}}(\zeta) \in \overline{F_{n}}$ for all $n \in \mathbb{N}$. Now suppose that $f$ is a quasimeromorphic mapping of transcendental type satisfying the hypotheses in the last part of the lemma. Since $\mathcal{J}(f)$ is backward invariant, we get that $\overline{G_{n}} \cap \mathcal{J}(f) \neq \varnothing$ for all $n \geq 0$. It follows that for all $n \geq 0$,

$$
f^{\ell_{n+1}}\left(\overline{G_{n}} \cap \mathcal{J}(f)\right) \supset \overline{F_{n+1}} \cap \mathcal{J}(f) .
$$

Now by applying the first part of the lemma to the closed sets $\overline{F_{n}} \cap \mathcal{J}(f)$, we deduce that $\zeta \in \mathcal{J}(f) \cap \overline{F_{0}}$ as required.

It should be noted that by setting $\ell_{n}=1$ for all $n \in \mathbb{N}$, we get a modified version of [93, Lemma 1]. This version shall be used for the proof of Theorem 5.1.1, while the general version shall be reserved for the proof of Theorem 5.1.2.

\subsubsection{A holding-up lemma for quasimeromorphic mappings with finitely many poles}

For a quasimeromorphic mapping with finitely many poles, it is possible to get sufficient conditions for the existence of a slow escaping point using the same 'holdingup' technique as that for quasiregular mappings of transcendental type. The proof of the following lemma is almost identical to that by Nicks [66, Lemma 3.1] and is therefore omitted.

Lemma 5.1.4. Let $f: \mathbb{R}^{d} \rightarrow \hat{\mathbb{R}}^{d}$ be a K-quasimeromorphic function of transcendental type with at least one pole. Let $p \in \mathbb{N}$ and, for $m \in \mathbb{N}$ and $i \in\{1,2, \ldots, p\}$, let $X_{m}^{(i)} \subset \mathbb{R}^{d}$ be non-empty bounded sets, with $X_{m}=\bigcup_{i=1}^{p} X_{m}^{(i)}$, such that

$$
\inf \left\{|x|: x \in X_{m}\right\} \rightarrow \infty \text { as } m \rightarrow \infty \text {. }
$$


(X1) for all $m \in \mathbb{N}$ and $i \in\{1,2, \ldots, p\}$, there exists some $j \in\{1,2, \ldots, p\}$ such that $f\left(X_{m}^{(i)}\right) \supset X_{m+1}^{(j)}$,

and there exists a strictly increasing sequence of integers $\left(m_{t}\right)$ such that

(X2) for all $t \in \mathbb{N}$ and $i \in\{1,2, \ldots, p\}$, there exists some $j \in\{1,2, \ldots, p\}$ such that $f\left(X_{m_{t}}^{(i)}\right) \supset X_{m_{t}}^{(j)}$, and

(X3) for all $t \in \mathbb{N}$ and $i \in\{1,2, \ldots, p\}, \overline{X_{m t}^{(i)}} \cap \mathcal{J}(f) \neq \varnothing$.

Then given any positive sequence $a_{n} \rightarrow \infty$, there exists $\zeta \in \mathcal{J}(f)$ and $N_{1} \in \mathbb{N}$ such that $\left|f^{n}(\zeta)\right| \rightarrow \infty$ as $n \rightarrow \infty$, while also $\left|f^{n}(\zeta)\right| \leq a_{n}$ whenever $n \geq N_{1}$.

\subsection{Proof of Theorem 5.1.1: Finitely many poles}

Using the growth result of Theorem 3.1.2 from Chapter 3, we are now in a position to prove Theorem 5.1.1 in the case where the quasimeromorphic mapping of transcendental type has at least one pole, but finitely many poles; this will closely follow the proof of Theorem 1.4.6 from [66]. There, the covering and waiting sets could be found sufficiently close to the essential singularity.

For $f: \mathbb{R}^{d} \rightarrow \hat{\mathbb{R}}^{d}$ a quasimeromorphic mapping of transcendental type with finitely many poles, there exists some $R>0$ such that all the poles of $f$ are contained in $B(0, R)$. This means that $f$ restricted to $A(R, \infty)$ is a quasiregular mapping with an essential singularity at infinity. It therefore suffices to verify that the results stated by Nicks in [66] for quasiregular mappings of transcendental type on $\mathbb{R}^{d}$ still hold for mappings defined on a neighbourhood of the essential singularity. 
Although some of the results are essentially those from [66], proofs will be given for those which require further arguments in the new setting.

\subsubsection{Functions with the pits effect}

Geometrically, a function $f$ is said to have the 'pits effect' if the image is 'large' except in 'small' domains, which form the so-called 'pits'. This concept was first described by Littlewood and Offord in [55] in the context of random entire functions on the complex plane.

Bergweiler and Nicks [17] adapted this idea and formalised their definition of the pits effect for quasiregular mappings of transcendental type using different notions of 'large' and 'small' as follows, written here for quasiregular mappings defined near an essential singularity.

Definition 5.2.1. Let $R>0$ and let $f: A(R, \infty) \rightarrow \mathbb{R}^{d}$ be a $K$-quasiregular mapping with an essential singularity at infinity. Then $f$ is said to have the pits effect if there exists some $N \in \mathbb{N}$ such that, for all $s>1$ and all $\varepsilon>0$, there exists $T_{0} \geq R$ such that

$$
\{x \in \overline{A(T, s T)}:|f(x)| \leq 1\}
$$

can be covered by $N$ balls of radius $\varepsilon T$ whenever $T>T_{0}$.

It was shown by Bergweiler and Nicks in [17, Theorem 8.1] that it is possible to replace the constant 1 in the condition $|f(x)| \leq 1$ by any positive value. By using Corollary 3.1.3 rather than [10, Lemma 3.4] in their proof, we get the following analogous result.

Lemma 5.2.2. Let $R>0$ and let $f: A(R, \infty) \rightarrow \mathbb{R}^{d}$ be a $K$-quasiregular mapping with an essential singularity at infinity that has the pits effect. Then there exists some $N \in \mathbb{N}$ such that, for all $s>1$, all $\alpha>1$ and all $\varepsilon>0$, there exists $T_{0} \geq R$ 
such that

$$
\left\{x \in \overline{A(T, s T)}:|f(x)| \leq T^{\alpha}\right\}
$$

can be covered by $N$ balls of radius $\varepsilon T$ whenever $T>T_{0}$.

Throughout the remainder of Section 5.2.1, we shall assume that $f$ is as in the statement of Theorem 5.1.1 and that the restriction of $f$ to the domain $A(R, \infty)$, with $R>0$, is a $K$-quasiregular mapping that has the pits effect. Now using Lemma 3.2.1, we can further assume that $R>0$ is sufficiently large that $M(r, f)$ is a strictly increasing function for $r \geq R$.

First we require some self-covering sets to achieve the 'hold-up' criteria from Lemma 5.1.4. The following lemma is essentially that of [66, Lemma 3.3], with the proof following similarly.

Lemma 5.2.3. There exists $\delta \in(0,1 / 2]$ and a sequence of points $x_{t} \rightarrow \infty$ such that the moduli $T_{t}=\left|x_{t}\right|$ are strictly increasing and the balls $B_{t}:=B\left(x_{t}, \delta T_{t}\right)$ are such that

$$
B_{t} \subset B\left(0,2 T_{t}\right) \subset f\left(B_{t}\right)
$$

for all $t \in \mathbb{N}$.

From Corollary 3.1.3, for all large $r$ we have $M(r, f)>2 r$. Thus we shall now assume that the $T_{t}$ as defined in Lemma 5.2.3 are large enough such that the sequence $\left(r_{t}\right)$, defined by $M\left(r_{t}, f\right)=T_{t}$ with $r_{t}>\max \{R, M(R, f)\}$, satisfies $M\left(r_{t}, f\right)>2 r_{t}$ for all $t \in \mathbb{N}$. Consequently, note that $\left(r_{t}\right)$ is a strictly increasing sequence with $r_{t} \rightarrow \infty$ as $t \rightarrow \infty$. We now have the following result, which is essentially that of [66, Lemma 3.4].

Lemma 5.2.4. For each $t \in \mathbb{N}$ and $\lambda \geq 2 T_{t}$,

$$
A\left(r_{t}, 2 \lambda\right) \subset f\left(A\left(r_{t}, \lambda\right)\right)
$$


Proof. Since $f$ has the pits effect and $T_{t}$ is large, then from Lemma 5.2.2, we claim that given any $\lambda \geq 2 T_{t}$, there exists $\lambda^{\prime} \in[(3 / 4) \lambda, \lambda]$ such that

$$
\inf \left\{|f(x)|:|x|=\lambda^{\prime}\right\} \geq 3 \lambda
$$

Suppose otherwise and let $N \in \mathbb{N}$ be as in Lemma 5.2.2. Then for all $\lambda^{\prime} \in[(3 / 4) \lambda, \lambda]$, there exists $x_{\lambda^{\prime}}$ such that $\left|x_{\lambda^{\prime}}\right|=\lambda^{\prime}$ and $\left|f\left(x_{\lambda^{\prime}}\right)\right|<3 \lambda$. As the length of the interval is $(1 / 4) \lambda$, then we find that the set

$$
\left\{x \in \overline{A\left(\frac{3}{4} \lambda, \lambda\right)}:|f(x)| \leq 3 \lambda\right\},
$$

cannot be covered by $N$ balls of radius $(1 / 12 N) \lambda$. Since $\lambda \geq 2 T_{t}$ and $T_{t}$ is large, then this set is contained in

$$
\left\{x \in \overline{A\left(\frac{3}{4} \lambda, \lambda\right)}:|f(x)| \leq\left(\frac{3}{4} \lambda\right)^{2}\right\}
$$

which contradicts Lemma 5.2.2 with $\alpha=2, s=4 / 3, \varepsilon=1 / 9 N$.

Now let $t \in \mathbb{N}$. Using equation (5.2.3), we have $f\left(\partial B\left(0, \lambda^{\prime}\right)\right) \cap B(0,3 \lambda)=\varnothing$. It then follows that

$$
A\left(r_{t}, 2 \lambda\right) \subset A(M(R, f), 3 \lambda) \subset f\left(A\left(R, \lambda^{\prime}\right)\right) \subset f(A(R, \lambda)) .
$$

Next let $y \in A\left(r_{t}, 2 \lambda\right)$. By equation (5.2.4), there exists an $x \in A(R, \lambda)$ such that $f(x)=y$. This gives 2 possibilities. If $x \in A\left(r_{t}, \lambda\right)$, then we are done. Otherwise if $|x| \in\left(R, r_{t}\right]$, then $|y|=|f(x)| \leq M\left(r_{t}, f\right)=T_{t}$. Thus by Lemma 5.2.3, there is some $w \in B_{t}$ such that $f(w)=y$. Finally, the definition of $B_{t}$ and $r_{t}$ yields

$$
B_{t} \subset A\left(\frac{T_{t}}{2}, \frac{3 T_{t}}{2}\right) \subset A\left(r_{t}, 2 T_{t}\right) \subset A\left(r_{t}, \lambda\right)
$$

as required. 
Using Lemma 5.2.3 and Lemma 5.2.4, we can appeal to Lemma 5.1.4, with $p=1$, to complete the proof of Theorem 5.1.1 for mappings with finitely many poles that have the pits effect. With this in mind, we shall omit the superscripts and choose the sets $X_{m}$ for each $m$.

Set $m_{1}=1$ and inductively define $m_{t+1}=m_{t}+K_{t}$, where $K_{t}>1$ is the smallest integer such that $(3 / 2) T_{t+1} \leq 2^{K_{t}} T_{t}$. Now for each $m \in \mathbb{N}$, set

$$
X_{m}=\left\{\begin{array}{cl}
B_{t} & \text { if } m=m_{t} \text { for some } t \in \mathbb{N} \\
A\left(r_{t}, 2^{m-m_{t}} T_{t}\right) & \text { if } m \in\left(m_{t}, m_{t+1}\right)
\end{array}\right.
$$

Firstly note that as $T_{t} \rightarrow \infty$ and $r_{t} \rightarrow \infty$ as $t \rightarrow \infty$, then equation (5.1.2) is satisfied. In addition, (X2) is satisfied due to equation (5.2.2) from Lemma 5.2.3. Next as $T_{t}$ are large, then appealing to Theorem 4.1.3(i) we can assume that $B\left(0,2 T_{t}\right) \cap \mathcal{J}(f) \neq \varnothing$. From this, equation (5.2.2) and Theorem 4.1.3(iii) then imply that $B_{t} \cap \mathcal{J}(f) \neq \varnothing$, so (X3) is satisfied. To show (X1) holds, we shall consider three cases:

(1) When $m=m_{t}$ for some $t \in \mathbb{N}$, then from equation (5.2.2),

$$
f\left(X_{m_{t}}\right)=f\left(B_{t}\right) \supset B\left(0,2 T_{t}\right) \supset A\left(r_{t}, 2 T_{t}\right)=X_{m_{t}+1} .
$$

(2) When $m \in\left(m_{t}, m_{t+1}-1\right)$ for some $t \in \mathbb{N}$, then by Lemma 5.2.4,

$$
f\left(X_{m}\right)=f\left(A\left(r_{t}, 2^{m-m_{t}} T_{t}\right)\right) \supset A\left(r_{t}, 2^{m+1-m_{t}} T_{t}\right)=X_{m+1}
$$

(3) When $m=m_{t+1}-1$ for some $t \in \mathbb{N}$, then by Lemma 5.2.4,

$$
\begin{aligned}
f\left(X_{m}\right) & =f\left(A\left(r_{t}, 2^{m_{t+1}-1-m_{t}} T_{t}\right)\right) \supset A\left(r_{t}, 2^{m_{t+1}-m_{t}} T_{t}\right) \\
& =A\left(r_{t}, 2^{K_{t}} T_{t}\right) \supset A\left(r_{t}, \frac{3 T_{t+1}}{2}\right) .
\end{aligned}
$$


Now since $T_{t+1} \geq T_{t}>2 r_{t}$ for all $t$, then

$$
f\left(X_{m}\right) \supset A\left(r_{t}, \frac{3 T_{t+1}}{2}\right) \supset A\left(\frac{T_{t+1}}{2}, \frac{3 T_{t+1}}{2}\right) \supset B_{t+1}=X_{m+1} .
$$

Finally, as all the hypotheses are satisfied, then an application of Lemma 5.1.4 completes the proof of Theorem 5.1.1 for mappings with finitely many poles that have the pits effect.

\subsubsection{Functions without the pits effect}

In this subsection, the primary objective is to prove Theorem 5.1.1 in the case where $f: \mathbb{R}^{d} \rightarrow \hat{\mathbb{R}}^{d}$ is a quasimeromorphic mapping of transcendental type with finitely many poles, whose restriction to a punctured neighbourhood of the essential singularity is a quasiregular mapping that does not have the pits effect. This will be done by adapting the methods found in [66, Section 3.4].

For $r>4 R>0$, we shall first define domains $Q_{\ell}(r) \subset A(R, \infty)$. In the following, we use the notation $r X:=\{r x: x \in X\}$. Further, recall that if $q$ is Rickman's constant from Theorem 1.2.6, then we call $q_{0}=q-1$ Rickman's quasiregular constant.

Let $q_{0}$ be Rickman's quasiregular constant and fix $2 q_{0}$ distinct unit vectors $\hat{u_{1}}, \hat{u_{2}}, \ldots$, $\hat{u_{2 q_{0}}}$, so each $\hat{u}_{\ell}$ is such that $\hat{u}_{\ell} \in \mathbb{R}^{d}$ and $\left|\hat{u}_{\ell}\right|=1$. Fix $\theta>0$ small enough so for all $\ell=1,2, \ldots, 2 q_{0}$, the truncated cones

$$
C_{\ell}=\left\{x \in A\left(\frac{1}{4}, 2 q_{0}+1\right): \frac{\hat{u}_{\ell} \cdot x}{|x|}>\cos (\theta)\right\}
$$

are such that $\overline{C_{\ell}} \cap \overline{C_{j}}=\varnothing$ for all pairs $\ell \neq j$, where $\hat{u_{\ell}} \cdot x$ denotes the scalar product. 
Now for $r>4 R$ and $\ell \in\left\{1,2, \ldots, 2 q_{0}\right\}$, define

$$
Q_{\ell}(r)=A\left(\ell r,\left(\ell+\frac{1}{2}\right) r\right) \cup r C_{\ell}
$$

A useful observation is that for all $\ell$ and $r, Q_{\ell}(r)=r Q_{\ell}(1)$ and that each $Q_{\ell}(1)$ is bounded away from infinity by the chordal metric; see Figure 5.1.

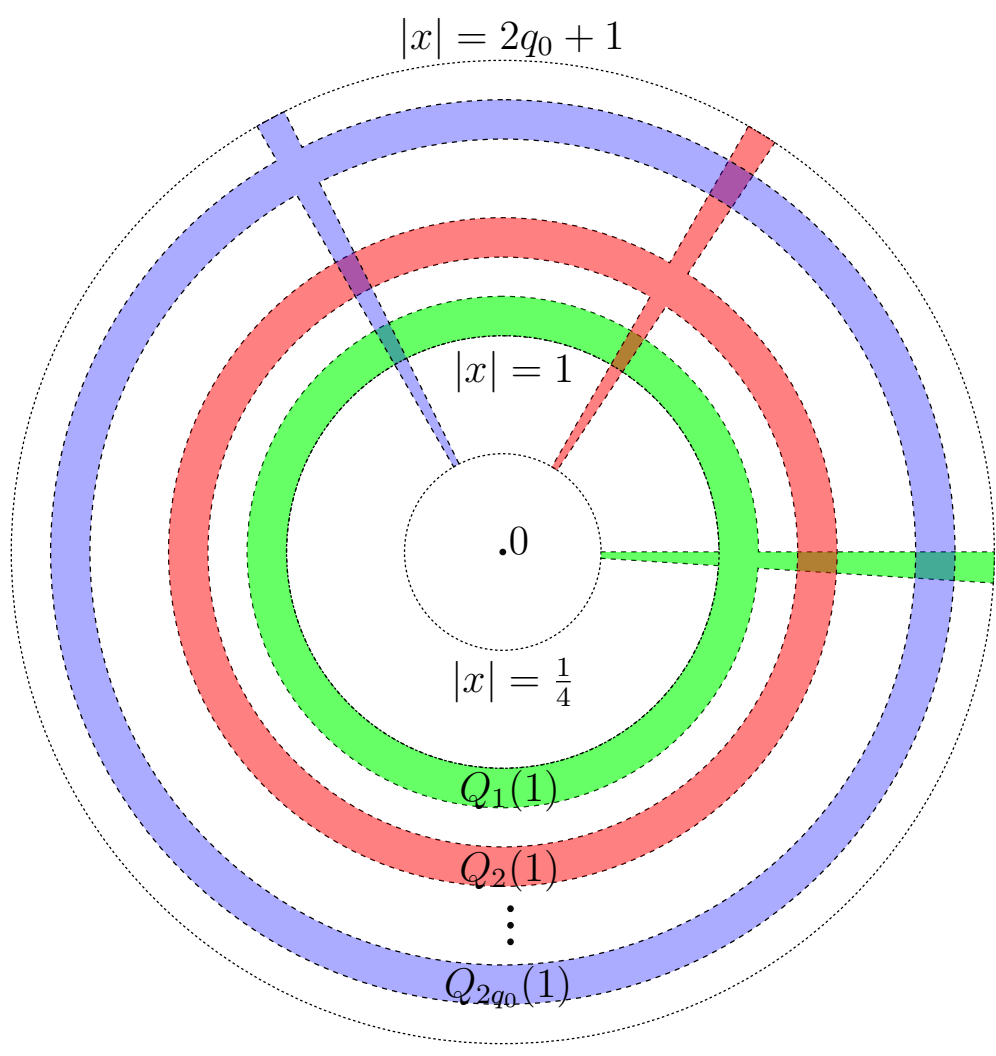

Figure 5.1: The sets $Q_{\ell}(1)$ for $\ell=1,2, \ldots, 2 q_{0}$ in dimension $d=2$.

By using a combinatorial argument, we can get a simple extension of Theorem 1.3.1. Here, we shall state the result for a family of $K$-quasiregular mappings, however the proof is analogous in the quasimeromorphic case.

Lemma 5.2.5. Let $\mathcal{F}$ be a family of $K$-quasiregular mappings on a domain $X \subset \mathbb{R}^{d}$ and let $q_{0}$ be Rickman's quasiregular constant. Let $N \in \mathbb{N}$ and, for $i=1,2, \ldots, N q_{0}$ and $n=1,2, \ldots, N$, let $A_{i, n}$ be bounded sets such that for each $n, \overline{A_{i, n}} \cap \overline{A_{j, n}}=\varnothing$ 
for all $i \neq j$.

Suppose that every $g \in \mathcal{F}$ omits a value from each set $\mathcal{A}_{i}=\bigcup_{n=1}^{N} A_{i, n}$. Then $\mathcal{F}$ is a normal family on $X$.

Proof. Fix an $N \in \mathbb{N}$ and for each $n \in\{1,2, \ldots, N\}$, let $\varepsilon_{n}>0$ be such that, for all $i \neq j$,

$$
\operatorname{dist}\left(A_{i, n}, A_{j, n}\right):=\inf \left\{\left|a_{i}-a_{j}\right|: a_{i} \in A_{i, n}, a_{j} \in A_{j, n}\right\} \geq \varepsilon_{n} .
$$

Set $\varepsilon=\min \left\{\varepsilon_{n}: n=1,2, \ldots, N\right\}$ and consider any set $D=\left\{d_{1}, d_{2}, \ldots, d_{N q_{0}}\right\}$, where $d_{i} \in \mathcal{A}_{i}$ for each $i$. It follows that there exists some $n \in\{1,2, \ldots, N\}$ such that $d_{i} \in \mathcal{A}_{i, n}$ for at least $q_{0}$ values of $i$; these $d_{i}$ form a subset $\left\{\alpha_{1}, \alpha_{2}, \ldots, \alpha_{q_{0}}\right\} \subset D$ such that $\left|\alpha_{k}-\alpha_{l}\right| \geq \varepsilon$ for $k \neq l$. Now by considering Theorem 1.3.1 and noting that each of the $A_{i, n}$ are bounded away from infinity in the chordal metric, we conclude that $\mathcal{F}$ is a normal family on $X$.

Note that in the above lemma, the result can be sharpened by asking that every mapping in $\mathcal{F}$ omits a value in at least $N\left(q_{0}-1\right)+1$ of the $\mathcal{A}_{i}$. We shall apply this lemma later with $N=2, A_{i, 1}=A(i, i+1 / 2)$ and $A_{i, 2}=C_{i}$, so that $\mathcal{A}_{i}=Q_{i}(1)$.

To find sets that satisfy the 'hold-up' criterion, we will first introduce some notation. Recall from equation (3.1.2) that the topological degree of $f$ at $y$ over a domain $D$ is denoted by $\mu(y, f, D)$. Then following Rickman $[87$, p. 80], we define

$$
A V(f, D):=\frac{1}{\omega_{d}} \int_{\hat{\mathbb{R}}^{d}} \frac{\mu(y, f, D)}{\left(1+|y|^{2}\right)^{d}} d y=\frac{1}{\omega_{d}} \int_{D} \frac{J_{f}(x)}{\left(1+|f(x)|^{2}\right)^{d}} d x,
$$

which is the average of $\mu(y, f, D)$ over all $y \in \hat{\mathbb{R}}^{d}$ with respect to the $d$-dimensional spherical measure. Here $\omega_{d}$ denotes the surface area of the unit $d$-sphere $S^{d}(0,1)$. It should be noted that Rickman identifies $\hat{\mathbb{R}}^{d}$ with $\left\{x \in \mathbb{R}^{d+1}:\left|x-(1 / 2) e_{d+1}\right|=1 / 2\right\}$, where $e_{k}$ denotes the $k^{\text {th }}$ unit vector, while we use $\left\{x \in \mathbb{R}^{d+1}:|x|=1\right\}$. This 
accounts for the differing factor of $2^{d}$ in the above definition. The following version of a result by Bergweiler [12, Theorem 3.2] shows that when the domain is a ball and the quasiregular mapping omits a sufficiently large set, then the average topological degree is bounded.

Theorem 5.2.6. Let $F \subset \mathbb{R}^{d}$ be such that $\operatorname{cap}(F)>0$, let $x \in \mathbb{R}^{d}$ and let $\alpha>1$. Then there exists a constant $C=C(d, F, \alpha)$, such that if $f: B(x, \alpha r) \rightarrow \mathbb{R}^{d} \backslash F$ is a K-quasiregular mapping, then

$$
A V(f, \overline{B(x, r)}) \leq C K(f)
$$

Now by utilising the average topological degree, we can give a criterion which states that if we have sufficiently many bounded domains such that the image of each one covers many of the others, then the closure of each domain must intersect the Julia set. This is an extension of [66, Lemma 2.5] to quasimeromorphic mappings of transcendental type.

Lemma 5.2.7. Let $f: \mathbb{R}^{d} \rightarrow \hat{\mathbb{R}}^{d}$ be a $K$-quasimeromorphic mapping of transcendental type with at least one pole. Let $p \in \mathbb{N}$ be such that $p>K(f)+q_{0}$, where $q_{0}$ is Rickman's quasiregular constant. Suppose that $W_{1}, W_{2}, \ldots, W_{p} \subset \mathbb{R}^{d}$ are bounded domains such that $\overline{W_{i}} \cap \overline{W_{j}}=\varnothing$ for all $i \neq j$, and for each $i \in\{1,2, \ldots, p\}$,

$$
f\left(W_{i}\right) \supset W_{j} \text { for at least } p-q_{0} \text { values of } j \in\{1,2, \ldots, p\} \text {. }
$$

Then $\overline{W_{i}} \cap \mathcal{J}(f) \neq \varnothing$ for all $i \in\{1,2, \ldots, p\}$.

Proof. Firstly, suppose that $\mathcal{J}(f) \cap \overline{W_{i}}=\varnothing$ for some $i \in\{1,2, \ldots, p\}$. Then $W_{i} \cap \mathcal{O}_{f}^{-}(\infty)=\varnothing$, so $f^{n}$ is $K$-quasiregular on $W_{i}$ for all $n \in \mathbb{N}$. Now note that for any $n \in \mathbb{N}$ then, counting multiplicity, $f^{n}\left(W_{i}\right)$ covers at least $\left(p-q_{0}\right)^{n}$ of the domains $W_{j}, j \in\{1,2, \ldots, p\}$. By setting $\nu=\left(p-q_{0}\right)^{n}$, there exist pairwise disjoint 
subsets $V_{1}, V_{2}, \ldots, V_{\nu}$ of $W_{i}$ such that if $m \in\{1,2, \ldots, \nu\}$, then $f^{n}\left(V_{m}\right)=W_{j}$ for some $j \in\{1,2, \ldots, p\}$. Hence for each $n \in \mathbb{N}$, there exists some $j \in\{1,2, \ldots, p\}$ such that

$$
\mu\left(y, f^{n}, W_{i}\right) \geq \frac{\nu}{p} \text { for all } y \in W_{j}
$$

This implies that there exists some constant $C_{1}>0$ such that for all $n \in \mathbb{N}$,

$$
A V\left(f^{n}, W_{i}\right) \geq \frac{C_{1} \nu}{p}
$$

Now as $\mathcal{J}(f) \cap \overline{W_{i}}=\varnothing$, then for each $x \in \overline{W_{i}}$ there exists some $\delta_{x}>0$ such that $B\left(x, 2 \delta_{x}\right) \cap \mathcal{J}(f)=\varnothing$ and $\hat{\mathbb{R}}^{d} \backslash \mathcal{O}_{f}^{+}\left(B\left(x, 2 \delta_{x}\right)\right)$ is infinite. As $E(f)$ is finite, then there exists a point $w \in \hat{\mathbb{R}}^{d} \backslash\left(\mathcal{O}_{f}^{+}\left(B\left(x, 2 \delta_{x}\right)\right) \cup E(f)\right)$. Since $\hat{\mathbb{R}}^{d} \backslash \mathcal{O}_{f}^{+}\left(B\left(x, 2 \delta_{x}\right)\right)$ is closed, then $\overline{\mathcal{O}_{f}^{-}(w)} \subset \hat{\mathbb{R}}^{d} \backslash \mathcal{O}_{f}^{+}\left(B\left(x, 2 \delta_{x}\right)\right)$. As $w \notin E(f)$, it follows by Theorem 4.1.4 and the definition of the forward orbit that cap $\left(\hat{\mathbb{R}}^{d} \backslash \mathcal{O}_{f}^{+}\left(B\left(x, 2 \delta_{x}\right)\right)\right)>0$.

Using Theorem 5.2.6 and equation (1.2.3), for each $x \in \overline{W_{i}}$ there exists some constant $C_{x}>0$, dependent on $x$, such that for all $n \in \mathbb{N}$,

$$
A V\left(f^{n}, B\left(x, \delta_{x}\right)\right) \leq C_{x} K\left(f^{n}\right) \leq C_{x} K(f)^{n}
$$

As $\overline{W_{i}}$ is compact and the union of $B\left(x, \delta_{x}\right)$ forms an open cover, then there exists a finite subcover of $\overline{W_{i}}$. Thus we get that there exists some constant $C_{2}>0$ such that

$$
A V\left(f^{n}, W_{i}\right) \leq C_{2} K(f)^{n}
$$

However as $p>K(f)+q_{0}$, then we get a contradiction from equations (5.2.6) and (5.2.7) when $n \in \mathbb{N}$ is large. The conclusion now follows.

Now by appealing to Lemma 3.2.1 and Corollary 3.1.3, throughout the remainder of Section 5.2.2 we assume without loss of generality that $R>0$ is sufficiently large 
such that the restriction $f: A(R, \infty) \rightarrow \mathbb{R}^{d}$ is a $K$-quasiregular mapping with an essential singularity at infinity that does not have the pits effect, and $M(r, f)$ is a strictly increasing function with $M(r, f)>r$ for all $r \geq R$.

The covering result will be based on that given in [66]; the proof follows analogously using the new growth result of Theorem 3.1.2 and Lemma 5.2.5.

Lemma 5.2.8. Let $q_{0}$ be Rickman's quasiregular constant and let $W_{1}, W_{2}, \ldots$, $W_{q_{0}} \subset \mathbb{R}^{d}$ be bounded sets such that $\overline{W_{i}} \cap \overline{W_{j}}=\varnothing$ for all pairs $i \neq j$. Then for all sufficiently large $r$ and each $\ell=1,2, \ldots, 2 q_{0}$, the following hold.

(C1) There exists some $j \in\left\{1,2, \ldots, 2 q_{0}\right\}$ such that $f\left(Q_{\ell}(r)\right) \supset Q_{j}(M(r, f))$.

(C2) There exists some $k \in\left\{1,2, \ldots, q_{0}\right\}$ such that $f\left(Q_{\ell}(r)\right) \supset M(r, f) W_{k}$.

The 'hold-up' lemma we will use is also closely based on [17, Section 3] (see also [66, Lemma 3.7]), with the proof following analogously using Lemma 5.2.5, Lemma 5.2.7 and Theorem 3.1.2

Lemma 5.2.9. Let $q_{0}$ be Rickman's quasiregular constant. Then there exist bounded domains $W_{1}, W_{2}, \ldots, W_{q_{0}} \subset\left\{x \in \mathbb{R}^{d}:|x| \geq 1 / 2\right\}$ satisfying $\overline{W_{i}} \cap \overline{W_{j}}=\varnothing$ for all pairs $i \neq j$, and a real sequence $T_{t} \rightarrow \infty$ with $T_{1}>4 R$ such that for every $t \in \mathbb{N}$ and $\ell \in\left\{1,2, \ldots, q_{0}\right\}$ the following hold.

(C3) There exists some $j \in\left\{1,2, \ldots, q_{0}\right\}$ such that $f\left(T_{t} W_{\ell}\right) \supset T_{t} W_{j}$.

(C4) For each $\alpha \in\left[4 R, M\left(T_{t}, f\right)\right]$, there exists some $k \in\left\{1,2, \ldots, 2 q_{0}\right\}$ such that $f\left(T_{t} W_{\ell}\right) \supset Q_{k}(\alpha)$.

(C5) $T_{t} \overline{W_{\ell}} \cap \mathcal{J}(f) \neq \varnothing$.

Now using Lemma 5.2.8 and Lemma 5.2.9, we shall once again appeal to Lemma 5.1.4 to complete the proof of Theorem 5.1.1 for mappings with finitely many poles that 
do not have the pits effect. This closely follows the construction technique in [66, Section 3.4].

Recall that $R>0$ is sufficiently large such that $f: A(R, \infty) \rightarrow \mathbb{R}^{d}$ is a $K$ quasiregular mapping with an essential singularity at infinity and $M(r, f)$ is a strictly increasing function with $M(r, f)>r$ for all $r \geq R$. Increasing $R$ if necessary, we may assume that $r \geq R$ is sufficiently large as in Lemma 5.2.8. Now for $p \in \mathbb{N} \cup\{0\}$, we define the iterated maximum modulus $M^{p}(r, f)$ as follows. Set $M^{0}(r, f)=r$ and $M^{1}(r, f)=M(r, f)$. Then for $p \geq 2$, iteratively define

$$
M^{p}(r, f)=M\left(M^{p-1}(r, f), f\right)
$$

We note that as $M(r, f)>r$ is strictly increasing on $r \geq R$, then the sequence $\left(M^{p}(r, f)\right)_{p=1}^{\infty}$ is strictly increasing for all $r \geq R$. In particular, these are well-defined for $f$.

Now towards the proof, take a real sequence $T_{t} \rightarrow \infty$ and bounded domains $W_{1}, W_{2}, \ldots, W_{q_{0}}$ as in Lemma 5.2.9. We may assume that $T_{1}>4 R$ and that $T_{t+1}>M\left(T_{t}, f\right)$. Then for each $t \in \mathbb{N}$, there exists a smallest integer $p_{t} \geq 2$ such that $M^{p_{t}}\left(T_{t}, f\right) \geq T_{t+1}$. By our choice of $p_{t}$, we have that $M^{p_{t}-1}(r, f)$ is continuous in $r$ and

$$
M^{p_{t}-1}\left(T_{t}, f\right) \leq T_{t+1} \leq M^{p_{t}}\left(T_{t}, f\right)=M^{p_{t}-1}\left(M\left(T_{t}, f\right), f\right)
$$

It follows from the intermediate value theorem that for each $t \in \mathbb{N}$, there exists some $\Upsilon_{t} \in\left[T_{t}, M\left(T_{t}, f\right)\right]$ such that $M^{p_{t}-1}\left(\Upsilon_{t}, f\right)=T_{t+1}$.

We now choose the sets $X_{m}^{(i)}$ for each $m \in \mathbb{N}$ and $i=1,2, \ldots, 2 q_{0}$ to satisfy Lemma 5.1.4 with $p=2 q_{0}$. Set $m_{1}=1$ and inductively define $m_{t+1}=m_{t}+p_{t}$, for $t \geq 1$. Now for each $m \in \mathbb{N}$ and for each $i=1,2, \ldots, 2 q_{0}$, set 


$$
X_{m}^{(i)}=\left\{\begin{array}{cl}
T_{t} W_{i} & \text { if } m=m_{t} \text { for some } t \in \mathbb{N}, i \leq q_{0} ; \\
T_{t} W_{1} & \text { if } m=m_{t} \text { for some } t \in \mathbb{N}, i>q_{0} \\
Q_{i}\left(M^{m-m_{t}-1}\left(\Upsilon_{t}, f\right)\right) & \text { if } m \in\left(m_{t}, m_{t+1}\right) .
\end{array}\right.
$$

Firstly note that as the $W_{i}$ and $T_{t}$ were chosen to be those from Lemma 5.2.9, then $T_{t}>4 R$ for each $t \in \mathbb{N}$ and $W_{i} \subset\left\{x \in \mathbb{R}^{d}:|x| \geq 1 / 2\right\}$ for each $i \in\left\{1,2, \ldots, 2 q_{0}\right\}$. This means that

$$
\inf \left\{|x|: x \in X_{m_{t}}\right\}=\inf \left\{|x|: x \in \bigcup_{i=1}^{2 q_{0}} T_{t} W_{i}\right\} \geq \frac{T_{t}}{2}
$$

Also by the definition of $Q_{i}(r)$, then for $m \in\left(m_{t}, m_{t+1}\right)$ we have

$$
\inf \left\{|x|: x \in X_{m}\right\}=\frac{M^{m-m_{t}-1}\left(\Upsilon_{t}, f\right)}{4} \geq \frac{\Upsilon_{t}}{4} \geq \frac{T_{t}}{4}
$$

Since $T_{t} \rightarrow \infty$ as $t \rightarrow \infty$, then equation (5.1.2) is satisfied. Further, observe that (X2) and (X3) are satisfied due to (C3) and (C5) from Lemma 5.2.9 respectively. Finally (X1) follows from (C1) and (C2) from Lemma 5.2.8, and (C4) from Lemma 5.2.9 as follows.

(1) When $m=m_{t}$ for some $t \in \mathbb{N}$, then by (C4), for each $i \in\left\{1,2, \ldots, q_{0}\right\}$, there exists some $j \in\left\{1,2, \ldots, 2 q_{0}\right\}$ such that

$$
f\left(X_{m_{t}}^{(i)}\right)=f\left(T_{t} W_{i}\right) \supset Q_{j}\left(\Upsilon_{t}\right)=X_{m_{t}+1}^{(j)}
$$

This also holds for each $i \in\left\{q_{0}+1, q_{0}+2, \ldots, 2 q_{0}\right\}$ as $X_{m_{t}}^{(i)}=T_{t} W_{1}=X_{m_{t}}^{(1)}$.

(2) When $m \in\left(m_{t}, m_{t+1}-1\right)$ for some $t \in \mathbb{N}$, then by using (C1), for each $i \in\left\{1,2, \ldots, 2 q_{0}\right\}$, there exists some $j \in\left\{1,2, \ldots, 2 q_{0}\right\}$ such that

$$
f\left(X_{m}^{(i)}\right)=f\left(Q_{i}\left(M^{m-m_{t}-1}\left(\Upsilon_{t}, f\right)\right)\right) \supset Q_{j}\left(M^{m-m_{t}}\left(\Upsilon_{t}, f\right)\right)=X_{m+1}^{(j)}
$$


(3) When $m=m_{t+1}-1$ for some $t \in \mathbb{N}$, note that

$$
X_{m}^{(i)}=Q_{i}\left(M^{m_{t+1}-m_{t}-2}\left(\Upsilon_{t}, f\right)\right)=Q_{i}\left(M^{p_{t}-2}\left(\Upsilon_{t}, f\right)\right)
$$

It then follows by (C2) that for each $i \in\left\{1,2, \ldots, 2 q_{0}\right\}$, there exists some $j \in\left\{1,2, \ldots, q_{0}\right\}$ such that

$$
\begin{aligned}
f\left(X_{m}^{(i)}\right)=f\left(Q_{i}\left(M^{p_{t}-2}\left(\Upsilon_{t}, f\right)\right)\right) & \supset M^{p_{t}-1}\left(\Upsilon_{t}, f\right) W_{j} \\
& =T_{t+1} W_{j}=X_{m_{t+1}}^{(j)}=X_{m+1}^{(j)} .
\end{aligned}
$$

As all the hypotheses are satisfied, then an application of Lemma 5.1.4 completes the proof of Theorem 5.1.1 for mappings with finitely many poles that do not have the pits effect.

\subsubsection{A covering result for functions without the pits effect}

Let $f: \mathbb{R}^{d} \rightarrow \hat{\mathbb{R}}^{d}$ be a quasimeromorphic function without the pits effect as in Section 5.2.2. By continuing to adopt the notation as in Section 5.2.2, we shall give a useful covering result regarding the sets $T_{t} W_{j}$ for use in Section 5.4.

Lemma 5.2.10. For $t \in \mathbb{N}$ and $j=1,2, \ldots, q_{0}$, let $T_{t}$ and $W_{j}$ be those from Lemma 5.2.9. By moving to a suitable subsequence of $\left(T_{t}\right)$, there exist constants $i_{0}, j_{0} \in\left\{1,2, \ldots, q_{0}\right\}$ such that for each $t \in \mathbb{N}$, there are subsets $Y_{t} \subset T_{2} W_{j_{0}}$ and $Z_{t} \subset T_{t+2} W_{i_{0}}$ where

$$
f^{c_{t}}\left(Y_{t}\right) \supset T_{t+2} W_{i_{0}} \text { and } f^{2}\left(Z_{t}\right) \supset T_{2} W_{j_{0}}
$$

for some $c_{t} \in \mathbb{N}$. Further, $Y_{t}$ and $Z_{t}$ can be chosen such that $f^{c_{t}}$ is continuous on $\bar{Y}_{t}$ and $f^{2}$ is continuous on $\bar{Z}_{t}$ for each $t \in \mathbb{N}$. 
Proof. Let $j \in\left\{1,2, \ldots, q_{0}\right\}$. By the construction after Lemma 5.2.9, it follows that for all $t \in \mathbb{N}$ there exists some $i_{j, t} \in\left\{1,2, \ldots, q_{0}\right\}$, some $c_{j, t} \in \mathbb{N}$ and some subset $Y_{j, t} \subset T_{2} W_{j}$ such that

$$
f^{c_{j, t}}\left(Y_{j, t}\right) \supset T_{t+2} W_{i_{j, t}}
$$

Since $i_{j, t}$ can only take values from a finite set, then by taking a suitable subsequence of $T_{t}$ and relabelling we can assume that $i_{j}=i_{j, t}$ is independent of $t \in \mathbb{N}$, so

$$
f^{c_{j, t}}\left(Y_{j, t}\right) \supset T_{t+2} W_{i_{j}}
$$

Next, observe that as $T_{2}>M\left(T_{1}, f\right)$, then there exists some $\alpha>T_{1}>4 R$ such that $M(\alpha, f)=T_{2}$. Then by (C4) from Lemma 5.2.9, for all $t \in \mathbb{N}$ there exists some $N_{j, t} \in\left\{1,2, \ldots, 2 q_{0}\right\}$ such that

$$
f\left(T_{t+2} W_{i_{j}}\right) \supset Q_{N_{j, t}}(\alpha)
$$

As $N_{j, t}$ can only take values from a finite set, then by taking another suitable subsequence of $T_{t}$ and relabelling, we can assume that $N_{j}=N_{j, t}$ is independent of $t$. This means that for all $t \in \mathbb{N}$,

$$
f\left(T_{t+2} W_{i_{j}}\right) \supset Q_{N_{j}}(\alpha) .
$$

Applying (C2) from Lemma 5.2.8, we get that there exists some $\ell \in\left\{1,2, \ldots, q_{0}\right\}$ such that

$$
f\left(Q_{N_{j}}(\alpha)\right) \supset M(\alpha, f) W_{\ell}=T_{2} W_{\ell}
$$

Combining equations (5.2.9) and (5.2.10), it now follows that there exists some 
subset $Z_{j, t} \subset T_{t+2} W_{i_{j}}$ such that

$$
f^{2}\left(Z_{j, t}\right) \supset T_{2} W_{\ell}
$$

By repeatedly applying the whole argument above, we can build a sequence of subscripts $\left(\ell_{n}\right)$ as follows. Set $\ell_{1}=1$. Then for each $n \geq 1$, let $\ell_{n+1}$ be the value of $\ell$ from equation (5.2.11) after applying the argument once to $T_{2} W_{\ell_{n}}$.

As $\ell_{n} \in\left\{1,2, \ldots, q_{0}\right\}$ for all $n \in \mathbb{N}$, then there will exist some smallest values $n_{1}, n_{2} \in \mathbb{N}$, with $n_{1}<n_{2}$, such that $\ell_{n_{1}}=\ell_{n_{2}}$. Let $\nu=\ell_{n_{2}-1}$. Using this, we set $j_{0}=\ell_{n_{1}}$ and $i_{0}=i_{\nu}$. Then using equations (5.2.8) and (5.2.11), for each $t \in \mathbb{N}$, set $Y_{t}=Y_{j_{0}, t}, Z_{t}=Z_{\nu, t}$ and $c_{t}=2\left(n_{2}-n_{1}-1\right)+\sum_{m=n_{1}}^{n_{2}-1} c_{\ell_{m}, t}$; see Figure 5.2.

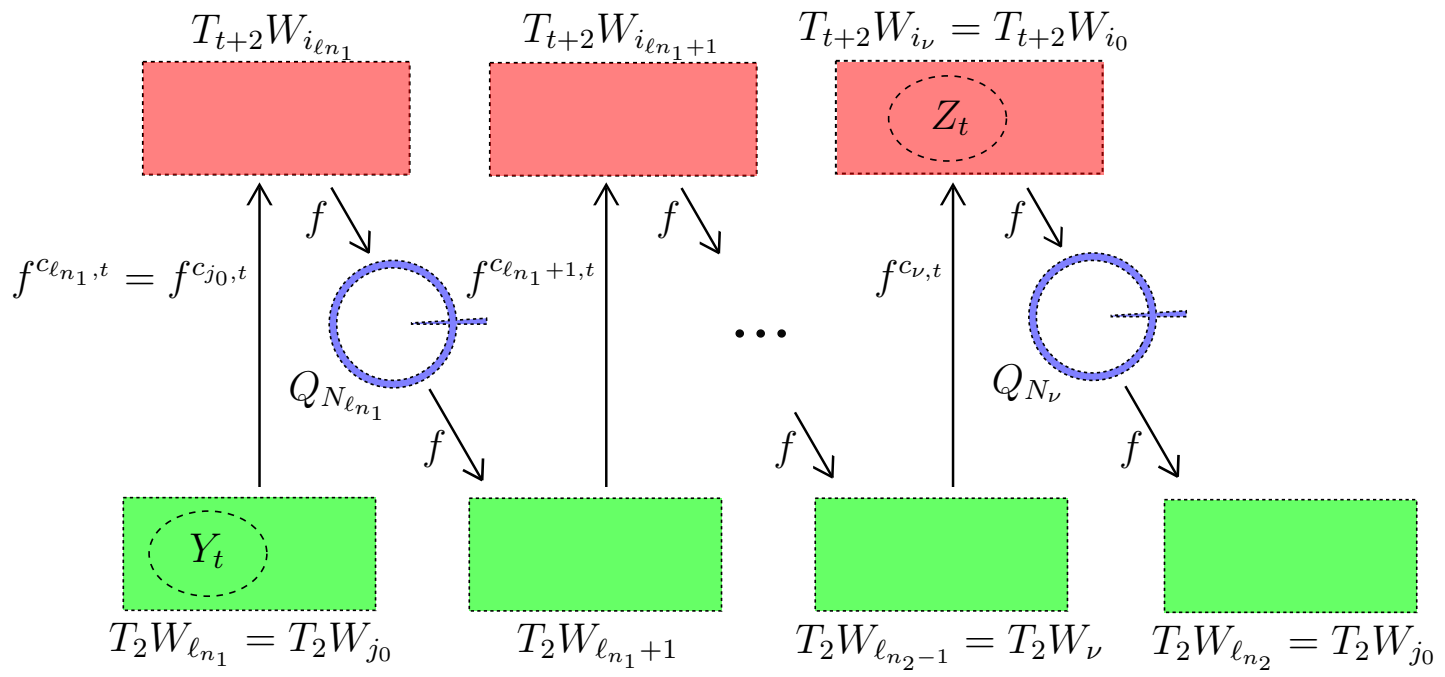

Figure 5.2: The sequence of covering sets and the construction of the sets $Y_{t} \subset T_{2} W_{j_{0}}$ and $Z_{t} \subset T_{t+2} W_{i_{0}}$ for each $t \in \mathbb{N}$.

It follows that there are some subsets $Y_{t} \subset T_{2} W_{j_{0}}$ and $Z_{t} \subset T_{t+2} W_{i_{0}}$ such that

$$
f^{c_{t}}\left(Y_{t}\right) \supset T_{t+2} W_{i_{0}} \text { and } f^{2}\left(Z_{t}\right) \supset T_{2} W_{j_{0}} \text {. }
$$

Finally, the lemma follows as $T_{1}>4 R$ implies that $f$ is continuous on each compact set $T_{t} \overline{W_{j}}$. 


\subsection{Proof of Theorem 5.1.1: Infinitely many poles}

In the case where $f$ has an infinite number of poles, it makes sense to utilise the neighbourhoods of the poles as a means of naturally approaching infinity. To this end, we seek a point that is able to 'pole-hop' between each neighbourhood and is able to return to the same neighbourhood after a finite number of steps via bounded sets. This idea is similar to that used by Rippon and Stallard in [93, Section 4], however the execution is quite different as it does not rely on the Ahlfors five island theorem.

The 'pole-hop' method creates a different situation to that found in the case of finitely many poles, where instead we relied on finding a point that could move forward at any time from any set. To achieve this modified 'hold-up' condition, we shall establish a different version of Lemma 5.1.4; see Figure 5.3.

To simplify notation, for $i \in \mathbb{N}$ and some fixed $p \in \mathbb{N}$, we shall denote the residue $i(\bmod p) \in\{0,1,2, \ldots, p-1\}$ as $[i]_{p}$. Here, note that $[1]_{p}+1=1$ if $p=1$, while $[1]_{p}+1=2$ otherwise.

Lemma 5.3.1. Let $f: \mathbb{R}^{d} \rightarrow \hat{\mathbb{R}}^{d}$ be a quasimeromorphic function of transcendental type with at least one pole. Let $p \in \mathbb{N}$ and for $m \in \mathbb{N}$ and $i \in\{1,2, \ldots, p\}$, let $X_{m}^{(i)} \subset \mathbb{R}^{d}$ be non-empty bounded sets, with $X_{m}=\bigcup_{i=1}^{p} X_{m}^{(i)}$, such that

$$
\inf \left\{|x|: x \in X_{m}\right\} \rightarrow \infty \text { as } m \rightarrow \infty
$$

Suppose further that

(X4) for all $m \in \mathbb{N}, f\left(X_{m}^{(1)}\right) \supset X_{m+1}^{(1)}$,

and there exists a strictly increasing sequence of integers $\left(m_{t}\right)$ such that 
(X5) for all $t \in \mathbb{N}$ and $i \in\{1,2, \ldots, p\}, f\left(X_{m_{t}}^{(i)}\right) \supset X_{m_{t}}^{\left([i]_{p}+1\right)}$, and

(X6) for all $t \in \mathbb{N}$ and $i \in\{1,2, \ldots, p\}, \overline{X_{m_{t}}^{(i)}} \cap \mathcal{J}(f) \neq \varnothing$.

Then given any positive sequence $a_{n} \rightarrow \infty$, there exists $\zeta \in \mathcal{J}(f)$ and $N_{1} \in \mathbb{N}$ such that $\left|f^{n}(\zeta)\right| \rightarrow \infty$ as $n \rightarrow \infty$, while also $\left|f^{n}(\zeta)\right| \leq a_{n}$ whenever $n \geq N_{1}$.

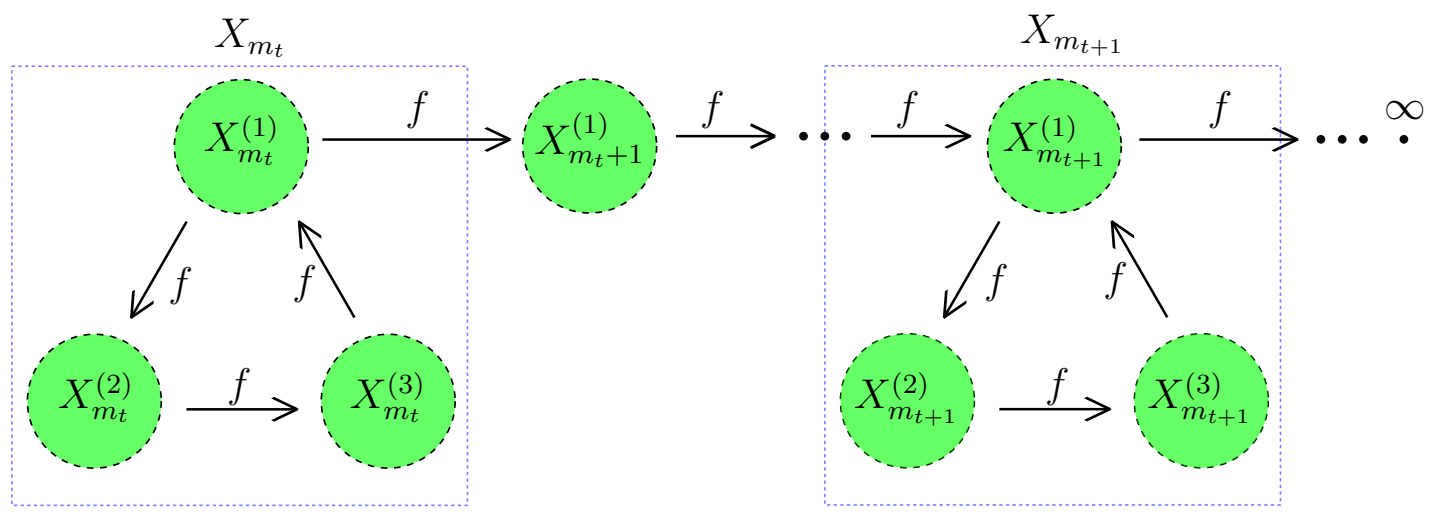

Figure 5.3: An example of the modified 'hold-up' condition for the case $p=3$.

Proof. Define an increasing real sequence $\left(\gamma_{m}\right)$ by

$$
\gamma_{m}=\sup \left\{|x|: x \in \bigcup_{j=1}^{m} X_{j}\right\}
$$

Since $a_{n} \rightarrow \infty$, then we can define a strictly increasing sequence of integers $N_{t}$ such that $\gamma_{m_{t}} \leq a_{n}$ for all $n \geq N_{t}$.

We shall now inductively define sets $F_{n}$, with $n \geq N_{1}$. Set $F_{N_{1}}=X_{m_{1}}^{(1)}$ and for each integer $n \geq N_{1}$, define

$$
F_{n+1}= \begin{cases}X_{m}^{\left([i]_{p}+1\right)} & \text { if } F_{n}=X_{m}^{(i)}, i \neq 1 \\ X_{m+1}^{(1)} & \text { if } F_{n}=X_{m}^{(1)}, m \neq m_{t} \\ X_{m_{t}+1}^{(1)} & \text { if } F_{n}=X_{m_{t}}^{(1)}, n \geq N_{t+1} \\ X_{m_{t}}^{\left([1]_{p}+1\right)} & \text { if } F_{n}=X_{m_{t}}^{(1)}, n<N_{t+1} .\end{cases}
$$


Firstly, observe that if $F_{n}=X_{m}^{(i)}$ with $i \neq 1$, then $m=m_{t}$ for some $t \in \mathbb{N}$. For supposing otherwise, then by construction there exists some natural number $1 \leq k<p$ such that $F_{n-k}=X_{m}^{(1)}$. If $m \neq m_{t}$ for any $t \in \mathbb{N}$, it follows that $F_{n-k+1}=X_{m+1}^{(1)}$. However, this is a contradiction since $n-k+1 \leq n$ and $F_{n}=X_{m}^{(i)}$, but $m+1>m$.

Now it follows from the construction, (X4) and (X5) that for each $n \geq N_{1}$, then $f\left(F_{n}\right) \supset F_{n+1}$. From this, together with (X6), an application of Lemma 5.1.3 gives us that there exists a point $\zeta_{N_{1}} \in \mathcal{J}(f) \backslash\{\infty\}$ whereby $f^{n-N_{1}}\left(\zeta_{N_{1}}\right) \in \overline{F_{n}}$ for all $n \geq N_{1}$.

Without loss of generality, we may assume that $\zeta_{N_{1}} \notin E(f)$. By applying Theorem 1.2.6 finitely many times and noting Theorem 4.1.3(iii), it follows that there exists $\zeta \in \mathcal{J}(f)$ such that $f^{N_{1}}(\zeta)=\zeta_{N_{1}}$. Therefore we have that $f^{n}(\zeta) \in \overline{F_{n}}$ for all $n \geq N_{1}$. Further, by equation (5.3.1) we have that $\left|f^{n}(\zeta)\right| \rightarrow \infty$ as $n \rightarrow \infty$.

To complete the proof, it remains to show that for all $n \geq N_{1}$, then $\left|f^{n}(\zeta)\right| \leq a_{n}$. Indeed, let $n \geq N_{1}$ be such that $F_{n}=X_{m_{1}}^{(i)}$ for some $i \in\{1,2, \ldots, p\}$. Then $F_{n} \subset X_{m_{1}}$ and so by equation (5.3.2) and the definition of $N_{1}$,

$$
\sup \left\{|x|: x \in F_{n}\right\} \leq \gamma_{m_{1}} \leq a_{n}
$$

We next aim to prove the following claim. Suppose that $n>N_{1}$ and $t \in \mathbb{N}$ are such that $m_{1} \leq m_{t}<m \leq m_{t+1}$ and $F_{n}=X_{m}^{(i)}$ for some $i \in\{1,2, \ldots, p\}$. Then $n \geq N_{t+1}$.

Indeed, if $i \neq 1$, then by a previous observation we must have $m=m_{t+1}$. This means there exists some natural number $k<p$ such that $F_{n-k}=X_{m}^{(1)}$ and $n-k>N_{1}$. Hence for any $i \in\{1,2, \ldots, p\}$, there exists some $N_{1}<n_{1} \leq n$ such that $F_{n_{1}}=X_{m}^{(1)}$. It follows by construction that either $F_{n_{1}-1}=X_{m-1}^{(1)}$ or $F_{n_{1}-1}=X_{m}^{(p)}$, where the latter case occurs only if $m=m_{t+1}$. As $m>m_{1}$, then by applying the above 
argument finitely many times, there must exist some integer $r \geq 0$ such that $F_{n_{1}-r p}=X_{m}^{(1)}$ and $F_{n_{1}-r p-1}=X_{m-1}^{(1)}$. It should be noted here that $n_{1}-r p>N_{1}$ as $m>m_{1}$. Hence there exists some $N_{1}<n_{2} \leq n_{1}$ such that $F_{n_{2}}=X_{m}^{(1)}$ and $F_{n_{2}-1}=X_{m-1}^{(1)}$.

As $F_{n_{2}}=X_{m}^{(1)}$ and $F_{n_{2}-1}=X_{m-1}^{(1)}$, then one of two cases may arise. If $m-1=m_{t}$, then this can only happen if $n_{2} \geq N_{t+1}$ by construction. Hence in this case, $n \geq N_{t+1}$

If $m-1 \neq m_{t}$, then by construction we can find some $N_{1} \leq n_{3}<n_{2}$ such that $F_{n_{3}}=X_{m_{t}}^{(1)}$ and $F_{n_{3}+1}=X_{m_{t}+1}^{(1)}$. However, this can only happen if $n_{3} \geq N_{t+1}$, so $n \geq N_{t+1}$ in this case; this proves the claim.

Now let $m, n$ and $t$ be as in the claim, so that $m_{1} \leq m_{t}<m \leq m_{t+1}$ and $F_{n}=X_{m}^{(i)}$ for some $i \in\{1,2, \ldots, p\}$. Since $m \leq m_{t+1}$, then we have

$$
F_{n} \subset \bigcup_{k=1}^{m_{t+1}} X_{k} .
$$

Hence by equation (5.3.2), the definition of $N_{t+1}$ and the fact that $n \geq N_{t+1}$, it follows that

$$
\sup \left\{|x|: x \in F_{n}\right\} \leq \gamma_{m_{t+1}} \leq a_{n}
$$

Finally, since for all $n \geq N_{1}$ we have that $F_{n}=X_{m}^{(i)}$ for some $m \geq m_{1}$ and $i \in\{1,2, \ldots, p\}$, it follows from equations (5.3.3) and (5.3.4) that $\left|f^{n}(\zeta)\right| \leq a_{n}$ as required.

To complete the proof of Theorem 5.1.1 for mappings with infinitely many poles, we shall apply Lemma 4.4.2 in the case when $N=1$.

Proof of Theorem 5.1.1: Infinitely many poles. Let $f$ have a sequence of poles $\left(x_{m}\right)$ tending to $\infty$. Now through Lemma 4.4.2 and choosing a subsequence of the poles and relabelling, we can construct the sequences $\left(R_{m}\right),\left(U_{m}\right)$ and $\left(E_{m}\right)$ by induction 
as follows.

Initialise $R_{1}=0$ and suppose that $R_{m}$ has been chosen for some $m \in \mathbb{N}$. By removing finitely many terms and relabelling, we may assume without loss of generality that $x_{m} \in A\left(R_{m}, \infty\right)$ and $x_{m}$ is not an exceptional point. Now set $U_{m}$ to be a bounded open neighbourhood of $x_{m}$, such that $\overline{U_{m}} \subset A\left(R_{m}, \infty\right)$ and $f^{-1}(u)$ is infinite for all $u \in \overline{U_{m}}$. By applying Lemma 4.4.2, choose a non-empty bounded open region $E_{m} \subset A\left(R_{m}, \infty\right)$ such that

$$
f\left(U_{m}\right) \supset \overline{E_{m}} \text {, and } f\left(E_{m}\right) \supset \overline{U_{m}} .
$$

Finally, choose $R_{m+1} \geq m+1$ such that $A\left(R_{m+1}, \infty\right) \subset f\left(U_{m}\right)$.

With $R_{m}, U_{m}$ and $E_{m}$ established, we shall now choose the sets $X_{m}^{(i)}$ that satisfy the hypotheses in Lemma 5.3.1 with $p=2$. For each $m \in \mathbb{N}$, define $X_{m}^{(1)}=U_{m}$ and $X_{m}^{(2)}=E_{m}$. Here, it should be noted that we are taking the subsequence $m_{t}=t$ for all $t \in \mathbb{N}$. Firstly, observe that equation (5.3.1) is satisfied since $\inf \left\{|x|: x \in U_{m} \cup E_{m}\right\} \geq R_{m}$ and $R_{m} \rightarrow \infty$ as $m \rightarrow \infty$.

Now as every $U_{m}$ is an open neighbourhood of a pole, then $U_{m} \cap \mathcal{J}(f) \neq \varnothing$. Also by equation (5.3.5) and Theorem 4.1.3(iii), then $E_{m} \cap \mathcal{J}(f) \neq \varnothing$ as well, so (X6) is satisfied. Further, (X5) is satisfied by equation (5.3.5) since for all $m \in \mathbb{N}$,

$$
f\left(X_{m}^{(1)}\right) \supset X_{m}^{(2)} \text {, and } f\left(X_{m}^{(2)}\right) \supset X_{m}^{(1)}
$$

To show (X4) is satisfied, observe that by construction,

$$
f\left(X_{m}^{(1)}\right)=f\left(U_{m}\right) \supset A\left(R_{m+1}, \infty\right) \supset U_{m+1}=X_{m+1}^{(1)} .
$$

Finally, an application of Lemma 5.3.1 completes the proof of Theorem 5.1.1 for functions with an infinite number of poles. 


\subsection{Proof of Theorem 5.1.2(i)}

\subsubsection{Sufficient conditions for Theorem 5.1.2(i)}

Let $f$ be a $K$-quasimeromorphic mapping of transcendental type with at least one pole. To prove Theorem 5.1.2(i), we shall provide sufficient conditions for the existence of infinitely many points in $B O(f) \cap \mathcal{J}(f)$ and $B U(f) \cap \mathcal{J}(f)$. Sets that satisfy these conditions will then be identified from each case of the proof of Theorem 5.1.1.

Firstly, suppose there exists some non-empty bounded set $U_{0}$ with $\overline{U_{0}} \cap \mathcal{J}(f) \neq \varnothing$ such that

(BO1) there exists some $N \in \mathbb{N} \cup\{0\}$ and bounded sets $U_{t}$ where $f\left(U_{N}\right) \supset U_{0}$ and if $N \geq 1$, then $f\left(U_{t}\right) \supset U_{t+1}$ for all $0 \leq t \leq N-1$.

Then by applying Lemma 5.1.3 with $F_{n}=U_{[n]_{N+1}}$ for all $n \in \mathbb{N}$, we get that there exists some $x \in \mathcal{J}(f) \cap B O(f) \cap \overline{U_{0}}$. By finding infinitely many such $U_{0}$ with pairwise disjoint closures, then we can conclude that $\mathcal{J}(f) \cap B O(f)$ is infinite.

Next, let $V$ be a non-empty bounded set and let $\left(k_{t}\right)$ be a sequence of natural numbers such that the following occurs:

(BU1a) for each $t \in \mathbb{N}$, there exists a non-empty bounded set $V_{t}$ and a subset $Y_{t} \subset V$ such that $f^{k_{t}}\left(Y_{t}\right) \supset V_{t}$ and $f^{k_{t}}$ is continuous on $\bar{Y}_{t}$;

(BU1b) for each $t \in \mathbb{N}$, there exists some subset $Z_{t} \subset V_{t}$ and some $m_{t} \in \mathbb{N}$ such that $f^{m_{t}}\left(Z_{t}\right) \supset V$ and $f^{m_{t}}$ is continuous on $\overline{Z_{t}}$; and

(BU2) inf $\left\{|x|: x \in V_{t}\right\} \rightarrow \infty$ as $t \rightarrow \infty$.

Then by applying Lemma 5.1 .3 with $G_{2 n-1}=Y_{n}$ and $G_{2 n}=Z_{n}, F_{2 n-1}=V$ and 
$F_{2 n}=V_{n}$ for all $n \in \mathbb{N}$, this gives a sufficient condition for the existence of a point $x \in B U(f)$. Moreover, if we have that

(BU3) $\mathcal{J}(f) \cap \overline{Y_{t}} \neq \varnothing$ for all $t \in \mathbb{N}$,

then Lemma 5.1.3 gives us a point $y \in \mathcal{J}(f) \cap B U(f) \cap \bar{V}$. Recalling Theorem 4.1.3(iii), it is clear that $f^{k}(y) \in \mathcal{J}(f) \cap B U(f)$ for all $k \in \mathbb{N}$, hence it follows that $\mathcal{J}(f) \cap B U(f)$ is infinite.

\subsubsection{Proof of Theorem 5.1.2(i)}

Let $f: \mathbb{R}^{d} \rightarrow \hat{\mathbb{R}}^{d}$ be a quasimeromorphic mapping of transcendental type with at least one pole but finitely many poles. As $f$ has finitely many poles then by taking $R>0$ sufficiently large, we have that $f: A(R, \infty) \rightarrow \mathbb{R}^{d}$ is a quasiregular mapping with an essential singularity at infinity. We shall first show that $B O(f) \cap \mathcal{J}(f)$ and $B U(f) \cap \mathcal{J}(f)$ are infinite when $f$ restricted to $A(R, \infty)$ has the pits effect. Indeed, by Lemma 5.2.3 and the arguments directly after Lemma 5.2.4, there exist bounded open balls $B_{t}, t \in \mathbb{N}$, such that

(i) $f\left(B_{t}\right) \supset B_{s}$ for all $s \leq t$;

(ii) there exists some sequence of natural numbers $\left(b_{t}\right)$ and some sets $Y_{t} \subset B_{1}$ such that for all $t \in \mathbb{N}, f^{b_{t}}\left(Y_{t}\right) \supset B_{t+1}$ and $f^{b_{t}}$ is continuous on $\bar{Y}_{t}$;

(iii) $\inf \left\{|x|: x \in B_{t}\right\} \rightarrow \infty$ as $t \rightarrow \infty$;

(iv) $\overline{B_{t}}$ are all pairwise disjoint; and

(v) $\overline{B_{t}} \cap \mathcal{J}(f) \neq \varnothing$ for all $t \in \mathbb{N}$.

(BO1) is clearly satisfied from (i) and (v), by setting $N=0$ and $U_{0}=B_{1}$. It then follows from (iv) that this can be repeated for each set $B_{t}, t \in \mathbb{N}$ to get infinitely many points. Therefore $B O(f) \cap \mathcal{J}(f)$ is infinite. 
Now set $V=B_{1}$ and $V_{t}=B_{t+1}$. Then (BU1a) and (BU1b) are satisfied by (i) and (ii), with $m_{t}=1$ and $k_{t}=b_{t}$ for all $t \in \mathbb{N}$. In addition, (BU2) is satisfied by (iii) and (BU3) is satisfied by (v) and the backward invariance of $\mathcal{J}(f)$. Therefore $B U(f) \cap \mathcal{J}(f)$ is infinite.

For the other cases, we can follow a similar argument. Indeed, suppose that $f$ is a quasimeromorphic mapping of transcendental type with at least one pole but finitely many, whose restriction to $A(R, \infty)$ for some $R>0$ is a quasiregular mapping that does not have the pits effect. Then from Lemma 5.2.9, the arguments immediately after Lemma 5.2.9, and Lemma 5.2.10, there are non-empty bounded sets $T_{t} W_{j}$ with $t \in \mathbb{N}$ and $j \in\left\{1,2, \ldots, q_{0}\right\}$, such that

(i) for each $t \in \mathbb{N}$ and $j \in\left\{1,2, \ldots, q_{0}\right\}$, there exists $i \in\left\{1,2, \ldots, q_{0}\right\}$ such that $f\left(T_{t} W_{j}\right) \supset T_{t} W_{i} ;$

(ii) there exists some constants $i_{0}, j_{0} \in\left\{1,2, \ldots, q_{0}\right\}$ such that for each $t \in \mathbb{N}$, there is some $c_{t} \in \mathbb{N}$, some subset $Y_{t} \subset T_{2} W_{j_{0}}$ and some subset $Z_{t} \subset T_{t+2} W_{i_{0}}$ whereby

$$
f^{c_{t}}\left(Y_{t}\right) \supset T_{t+2} W_{i_{0}} \text { and } f^{2}\left(Z_{t}\right) \supset T_{2} W_{j_{0}}
$$

with $f^{c t}$ continuous on $\overline{Y_{t}}$ and $f^{2}$ continuous on $\bar{Z}_{t}$;

(iii) $\inf \left\{|x|: x \in \cup_{j} T_{t} W_{j}\right\} \rightarrow \infty$ as $t \rightarrow \infty$;

(iv) $T_{t} \overline{W_{j}}$ are all pairwise disjoint; and

(v) $T_{t} \overline{W_{j}} \cap \mathcal{J}(f) \neq \varnothing$ for all $t \in \mathbb{N}$ and $j \in\left\{1,2, \ldots, q_{0}\right\}$.

Now fix some $t \in \mathbb{N}$. As $i \in\left\{1,2, \ldots, q_{0}\right\}$, then applying (i) sufficiently many times we have that there exists $N \in \mathbb{N} \cup\{0\}$ and $i_{0}, i_{1}, \ldots, i_{N} \in\left\{1,2, \ldots, q_{0}\right\}$ such that $f\left(T_{t} W_{i_{t}}\right) \supset T_{t} W_{i_{t+1}}$ and $f\left(T_{t} W_{i_{N}}\right) \supset T_{t} W_{i_{0}}$. This means that (BO1) is satisfied with $U_{t}=T_{t} W_{i_{t}}$. It then follows from (iv) and (v) that $B O(f) \cap \mathcal{J}(f)$ is infinite. 
Next, using (ii) set $V=T_{2} W_{j_{0}}$ and for each $t \in \mathbb{N}$ set $V_{t}=T_{t+2} W_{i_{0}}$. It then follows from (ii) that (BU1a) and (BU1b) are satisfied with sets $Y_{t}, Z_{t}$ and sequences $k_{t}=c_{t}$ and $m_{t}=2$ for each $t \in \mathbb{N}$. Further, (BU2) is given by (iii) whilst (BU3) follows from (v) and the backward invariance of $\mathcal{J}(f)$. Hence $B U(f) \cap \mathcal{J}(f)$ is infinite in this case.

When $f$ has infinitely many poles, then for $t, m \in \mathbb{N}$ we can choose neighbourhoods of poles $D_{t}$ and use Lemma 4.4.2 to get non-empty bounded sets $E_{t, m}$ such that

(i) for each fixed $t \in \mathbb{N}$, we have $f\left(D_{t}\right) \supset \overline{E_{t, m}}$ and $f\left(E_{t, m}\right) \supset \overline{D_{t}}$ for all $m \in \mathbb{N}$;

(ii) for each fixed $t \in \mathbb{N}$, then $\inf \left\{|x|: x \in E_{t, m}\right\} \rightarrow \infty$ as $m \rightarrow \infty$;

(iii) $\overline{D_{t}}$ are all pairwise disjoint and $\overline{E_{t, m}}$ are all pairwise disjoint; and

(iv) for each $t, m \in \mathbb{N}$, we have $\overline{D_{t}} \cap \mathcal{J}(f) \neq \varnothing$ and $\overline{E_{t, m}} \cap \mathcal{J}(f) \neq \varnothing$.

For each $t \in \mathbb{N}$, setting $U_{0}=D_{t}$ and $U_{1}=E_{t, 1}$ satisfies (BO1) by (i). It then follows by (iii) that $B O(f) \cap \mathcal{J}(f)$ is infinite.

Further, set $V=D_{1}$ and $V_{m}=E_{1, m}$. Then (BU1)-(BU3) all follow from (i), (ii) and (iv) respectively, thus $B U(f) \cap \mathcal{J}(f)$ is infinite. This completes the proof of Theorem 5.1.2(i).

\subsection{Proof of Theorem 5.1.2(ii)}

In proving Theorem 5.1.2(ii), the inclusion will be attained as a corollary to the following result, which is similar to that from [93, Lemma 10].

Lemma 5.5.1. Let $f: \mathbb{R}^{d} \rightarrow \hat{\mathbb{R}}^{d}$ be a quasimeromorphic mapping of transcendental type with at least one pole. Suppose that there is an infinite set $X \subset \mathbb{R}^{d}$ such that $X$ is completely invariant under $f$ and $\mathbb{R}^{d} \backslash\left(X \cup \mathcal{O}_{f}^{-}(\infty)\right)$ is infinite. Then $\mathcal{J}(f) \subset \partial X$. 
Proof. Let $x \in \mathcal{J}(f)$ and let $U_{x}$ be an arbitrary neighbourhood of $x$. Since $X$ and $\mathbb{R}^{d} \backslash\left(X \cup \mathcal{O}_{f}^{-}(\infty)\right)$ are infinite sets, then $X \backslash E(f)$ and $\mathbb{R}^{d} \backslash\left(X \cup \mathcal{O}_{f}^{-}(\infty) \cup E(f)\right)$ are non-empty. Now $X$ and $\mathbb{R}^{d} \backslash\left(X \cup \mathcal{O}_{f}^{-}(\infty)\right)$ are both completely invariant, so by Theorem 4.1.3(vi) it follows that $X \cap U_{x} \neq \varnothing$ and $\left(\mathbb{R}^{d} \backslash\left(X \cup \mathcal{O}_{f}^{-}(\infty)\right)\right) \cap U_{x} \neq \varnothing$. As $X$ and $\mathbb{R}^{d} \backslash\left(X \cup \mathcal{O}_{f}^{-}(\infty)\right)$ are disjoint, then we must have $\partial X \cap U_{x} \neq \varnothing$. Finally, since $U_{x}$ was arbitrary, then $x \in \partial X$ as required.

Since $I(f), B O(f)$ and $B U(f)$ are all completely invariant and disjoint, then the result follows from Theorem 5.1.2(i).

\subsubsection{Counterexamples}

To show that the reverse inclusion in Theorem 5.1.2 does not necessarily hold, we shall first construct a mapping similar to those found in [17, Example 7.3] and [24, Example 1]. This will be done by introducing poles in the function in such a way that the key dynamical properties we want to exploit are retained.

The first mapping $f: \mathbb{R}^{2} \rightarrow \mathbb{R}^{2}$ will be such that $(\partial I(f) \cap \partial B O(f)) \backslash \mathcal{J}(f) \neq \varnothing$, where we identify $\mathbb{C}$ with $\mathbb{R}^{2}$ in the usual way.

Example 5.5.2. Let $h: \mathbb{C} \rightarrow \hat{\mathbb{C}}$ be the transcendental meromorphic function defined by $h(z)=2+\exp (-z)+(z+1)^{-1}$, and define $g: \mathbb{C} \rightarrow \hat{\mathbb{C}}$ by $g(z)=z+h(z)$. Firstly, note that if $z$ is in the right half-plane $H_{1}:=\{z: \operatorname{Re}(z)>1\}$, then $h(z) \in\{v: 1<\operatorname{Re}(v)<3\}$. Now, we have $g\left(H_{1}\right) \subset H_{1}$ and $g^{n}(z) \rightarrow \infty$ as $n \rightarrow \infty$ whenever $z \in H_{1}$.

Next, for a large constant $M \in \mathbb{R}$ define $f: \mathbb{C} \rightarrow \hat{\mathbb{C}}$ by

$$
f(z)= \begin{cases}g(z) & \text { if } \operatorname{Re}(z) \leq M \text { or } \operatorname{Re}(z) \geq 2 M \\ g(z)+h(z) \sin \left(\frac{\pi \operatorname{Re}(z)}{M}\right) & \text { if } M<\operatorname{Re}(z)<2 M\end{cases}
$$

To see that $f$ is a quasimeromorphic mapping of transcendental type with one 
pole when $M$ is large, first recall Example 1.2.5 where some sufficient conditions were given for when the pointwise summation of a quasiregular mapping and a continuous mapping is quasiregular in the complex plane. In short, this occurs when one of the mappings sufficiently 'dominates' the other with respect to their partial derivatives, whilst being absolutely continuous on (almost all) lines and sufficiently smooth almost everywhere.

Firstly note that $f$ is continuous on the whole of $\mathbb{C}$ and is meromorphic on the region $\mathbb{C} \backslash\{z: M<\operatorname{Re}(z)<2 M\}$. Also for $z \in H_{1}$, we have $\left|g_{z}\right|=\mid 1-\exp (-z)-$ $(z+1)^{-2} \mid \leq 3$ as $\operatorname{Re}(z) \geq 1$.

Now consider the domain $X:=\{z: M<\operatorname{Re}(z)<2 M\}$ and set $q(z)=$ $h(z) \sin (\pi \operatorname{Re}(z) / M)$, which is continuous there. Let $z=x+i y$, where $x, y \in \mathbb{R}$. Then

$$
\begin{aligned}
\left|q_{x}\right| & =\left|\left(-\exp (-z)-(z+1)^{-2}\right) \sin \left(\frac{\pi \operatorname{Re}(z)}{M}\right)+\frac{\pi}{M} h(z) \cos \left(\frac{\pi \operatorname{Re}(z)}{M}\right)\right| \\
& \leq\left(1+\frac{\pi}{M}\right)\left(\exp (-M)+(M+1)^{-2}\right)+\frac{2 \pi}{M} \\
\left|q_{y}\right| & =\left|\left(-i \exp (-z)-i(z+1)^{-2}\right) \sin \left(\frac{\pi \operatorname{Re}(z)}{M}\right)\right| \leq \exp (-M)+(M+1)^{-2}, \text { and } \\
\left|g_{z}\right| & \geq 1-\left|\exp (-z)-(z+1)^{-2}\right| \geq 1-\left(\exp (-M)+(M+1)^{-2}\right)
\end{aligned}
$$

From this, we can see that as $M$ increases then $\left|q_{x}\right|$ and $\left|q_{y}\right|$ tend to 0 , while $\left|g_{z}\right|$ tends to 1 . Further, as $q_{x}$ and $q_{y}$ are continuous in $X$, then $q$ is continuously realdifferentiable, and hence $q \in W_{2, l o c}^{1}(X)$. Consequently when $M$ is sufficiently large, we can conclude from Example 1.2.5 that $f$ is quasiregular on $X$. Finally as $f$ is continuous on $\mathbb{C}, \partial X$ has zero measure, and $f$ has bounded partial derivatives on $H_{1} \backslash \partial X$, it follows that $f$ is a quasimeromorphic mapping of transcendental type on the whole of $\mathbb{C}$ with one pole.

For the dynamics of $f$, first note that $f\left(H_{1}\right) \subset H_{1}$, so $H_{1} \cap \mathcal{J}(f)=\varnothing$. Also, the 
point $w=3 M / 2$ is such that $f(w)=w$, while $f(x)>x$ for all real $x>w$. This means that $f^{n}(x) \rightarrow \infty$ as $n \rightarrow \infty$ for all real $x>w$, thus $(w, \infty) \subset I(f)$ and $w \in B O(f)$. Therefore $w \in(\partial I(f) \cap \partial B O(f)) \backslash \mathcal{J}(f)$.

This example can be extended to a quasimeromorphic mapping of transcendental type $\tilde{f}: \mathbb{C} \rightarrow \hat{\mathbb{C}}$ with infinitely many poles, by replacing $h$ with $\tilde{h}: \mathbb{C} \rightarrow \hat{\mathbb{C}}$ defined by

$$
\tilde{h}(z)=2+\exp (-z)+\sum_{k=1}^{\infty}\left(z+2^{k}-1\right)^{-1}
$$

and replacing $g$ with $\tilde{g}: \mathbb{C} \rightarrow \hat{\mathbb{C}}$ defined by $\tilde{g}(z)=z+\tilde{h}(z)$. Here, as $\left|z+2^{k}-1\right|>2^{k}$ for all $z \in H_{1}$, then $\tilde{h}(z) \in\{v: 1 / 2<\operatorname{Re}(v)<7 / 2\}$ on $H_{1}$. This means that the behaviour of $H_{1}$ and $w=3 M / 2$ under $\tilde{g}$, hence also for $\tilde{f}$, remains the same as above. A similar argument as above can be applied to show that $\tilde{f}$ is indeed quasimeromorphic of transcendental type on the whole of $\mathbb{C}$.

The final example is a direct modification of the example constructed in [71], as we will only require specific dynamics in the upper half plane to find a point in $\partial B U(f) \backslash \mathcal{J}(f)$; see also [14, Section 6].

Example 5.5.3. Let $h: \mathbb{C} \rightarrow \mathbb{C}$ be the quasiconformal mapping constructed in [71, Proof of Theorem 4]. This map is such that $B U(h)$ and $B O(h)$ intersect the upper half-plane $\mathbb{H}:=\{z: \operatorname{Im}(z)>0\}$ non-trivially, and $h(\mathbb{H}) \subset \mathbb{H}$.

Next for a small constant $\alpha>0$, let $g: \mathbb{C} \rightarrow \hat{\mathbb{C}}$ be defined by

$$
g(z)= \begin{cases}z & \text { if } \operatorname{Im}(z) \geq 0 \\ z-\alpha(\operatorname{Im}(z))\left(\exp \left(-z^{2}\right)+(z+4 i)^{-1}\right) & \text { if } \operatorname{Im}(z) \in[-1,0) \\ z+\alpha\left(\exp \left(-z^{2}\right)+(z+4 i)^{-1}\right) & \text { otherwise. }\end{cases}
$$

As in Example 5.5.2, it is possible to show through direct calculation that if $\alpha$ is sufficiently small, then $g$ is a quasimeromorphic mapping of transcendental type with one pole. Note that $g$ is the identity mapping on the upper half-plane, so 
$g(\mathbb{H}) \subset \mathbb{H}$.

Now the mapping $f:=g \circ h$ is also a quasimeromorphic mapping of transcendental type with one pole. It follows that $f(\mathbb{H}) \subset \mathbb{H}$ and so $\mathcal{J}(f) \cap \mathbb{H}=\varnothing$. Further since $g$ is the identity mapping on $\mathbb{H}$, then $f$ has the same dynamics on $\mathbb{H}$ as $h$. This means that $\mathbb{H} \cap B U(f) \neq \varnothing$ and $\mathbb{H} \cap B O(f) \neq \varnothing$. As $B O(f)$ and $B U(f)$ are disjoint, then $\mathbb{H} \cap \partial B U(f) \neq \varnothing$, hence $\partial B U(f) \backslash \mathcal{J}(f) \neq \varnothing$ as required.

By making a simple modification, we can also create a quasimeromorphic mapping of transcendental type with infinitely many poles, by replacing $(z+4 i)^{-1}$ in the definition of $g(z)$ with $\sum_{k=1}^{\infty}\left(z+2^{k}+4 i\right)^{-1}$; the dynamics of the new function remain unchanged in $\mathbb{H}$ and hence the result follows. 


\section{$6 \quad$ Quasimeromorphic mappings with countably many essential singularities}

In this chapter, we shall aim to define and study a generalisation of the Bolsch class $\mathbb{S}$, defined in Section 1.5, for quasimeromorphic mappings, and develop natural analogues of some results found in Chapters 2 - 5. We will define and study the Julia set for mappings in this class and show that it exhibits analogous properties to those listed in Theorem 4.1.3. By following Dominguez, Montes de Oca and Sienra in [25], we shall define and explore several escaping sets. Here we show they have similar properties to the escaping set for quasimeromorphic mappings, including the existence of points escaping arbitrarily slowly to each essential singularity. We finally answer three questions posed in [25] regarding the existence of points for which all subsequences of their iterates have specific properties.

\subsection{Introduction}

Recall from Section 1.5 that when studying the Fatou-Julia theory of meromorphic functions on the complex plane, it is useful to consider the smallest class of functions closed under composition that contains them. This class, denoted by $\mathbb{S}$, 
was established and studied by Bolsch in [20, 22], and is called the Bolsch class; see also [25]. Further generalisations of the Bolsch class have been considered in $[4,42]$

It is known that the composition of two quasimeromorphic mappings is not in general a quasimeromorphic mapping, due to poles becoming essential singularities for the composed map. Therefore to study their dynamical behaviour in more depth, we shall consider an analogue of the Bolsch class with respect to quasimeromorphic mappings, which we shall denote as $\mathbb{S}_{q m}$.

Definition 6.1.1. Let $d \geq 2$ be fixed. Then the ( $d$-dimensional) quasimeromorphic Bolsch class $\mathbb{S}_{q m}$ is defined as

$$
\begin{aligned}
& \mathbb{S}_{q m}=\left\{f: \text { there exists a closed countable set } A(f) \subset \hat{\mathbb{R}}^{d} \text { such that } f\right. \text { is } \\
&\text { quasimeromorphic on } \left.\hat{\mathbb{R}}^{d} \backslash A(f) \text { but in no larger set }\right\} .
\end{aligned}
$$

Similar to the Bolsch class $\mathbb{S}$, by adapting the proof of Theorem 1.5.2(i) it is clear that for $f \in \mathbb{S}_{q m}$, every point of $A(f)$ is the limit of isolated essential singularities of $f$. Furthermore, it can be shown that the quasimeromorphic Bolsch class $\mathbb{S}_{q m}$ is indeed a class closed under composition; the proof of this shall be deferred to Section 6.2 as we need a version of Theorem 1.2.6 in the new setting. We shall now consider a few special cases of mappings $f \in \mathbb{S}_{q m}$, whose Fatou-Julia theory has already been studied.

If $A(f)=\varnothing$ then $f$ is a quasiregular self-map of $\hat{\mathbb{R}}^{d}$, which has been studied by Bergweiler in [12]. If $A(f)$ is non-empty, then by normalising $f$ via a suitable Möbius map, we can assume without loss of generality that $\infty \in A(f)$.

Now suppose $A(f)=\{\infty\}$. If infinity is omitted in the image, then $f$ is a quasiregular mapping of transcendental type, whose Julia set was defined and studied by Bergweiler and Nicks in [17]. Otherwise, $f^{-1}(A(f))$ is non-empty and thus the 
backward orbit of $A(f)$, denoted $\mathcal{O}_{f}^{-}(A(f))$, contains at least two points.

Collecting these cases together, we get the following subsets of $\mathbb{S}_{q m}$ :

$$
\begin{aligned}
\mathcal{P}:= & \left\{f: \hat{\mathbb{R}}^{d} \rightarrow \hat{\mathbb{R}}^{d}: f \text { is a quasiregular map }\right\} ; \\
\mathcal{T}:= & \left\{f: \mathbb{R}^{d} \rightarrow \mathbb{R}^{d}: f \text { is a quasiregular map of transcendental type }\right\} ; \\
\mathcal{Q} \mathcal{M}_{\text {fin }}:= & \left\{f: \hat{\mathbb{R}}^{d} \backslash A(f) \rightarrow \hat{\mathbb{R}}^{d}: f\right. \text { is a quasimeromorphic map with } \\
& \left.\infty \in A(f) \text { and card }\left(\mathcal{O}_{f}^{-}(A(f))\right) \geq 2 \text { is finite }\right\} ; \text { and } \\
\mathcal{Q} \mathcal{M}_{\infty}:= & \left\{f: \hat{\mathbb{R}}^{d} \backslash A(f) \rightarrow \hat{\mathbb{R}}^{d}: f\right. \text { is a quasimeromorphic map with } \\
& \left.\infty \in A(f) \text { and } \mathcal{O}_{f}^{-}(A(f)) \text { is infinite }\right\} .
\end{aligned}
$$

We note that $\mathcal{P}, \mathcal{T}$ and $\mathcal{Q} \mathcal{M}_{\infty}$ are closed under composition and hence form classes. However, $\mathcal{Q} \mathcal{M}_{\text {fin }}$ is not closed under composition.

Example 6.1.2. Let $f: \mathbb{R}^{3} \rightarrow \hat{\mathbb{R}}^{3}$ be the mapping constructed in the proof of Corollary 2.1.3 and let $M: \hat{\mathbb{R}}^{3} \rightarrow \mathbb{R}^{3}$ be the Möbius map given by $M(x)=x+a$, where $a \in \mathbb{R}^{d}$ is such that $f^{-2}(a)$ is infinite. Then $F=f^{2}$ and $G=M \circ f^{2} \circ M^{-1}$ are in $\mathcal{Q} \mathcal{M}_{\text {fin }}$, with $A(F)=\{0, \infty\}$ and $A(G)=\{a, \infty\}$. However, $A(G \circ F)$ is infinite, hence $G \circ F \in \mathcal{Q M}_{\infty}$.

Recall that the theory used to study mappings in $\mathcal{P}$ and $\mathcal{T}$ is quite different to the theory used for quasimeromorphic mappings of transcendental type with at least one pole. As this new class is being studied with respect to the latter quasimeromorphic mappings, we shall assume henceforth that $A(f)$ and $f^{-1}(A(f))$ are non-empty, unless otherwise specified. This pertains to studying mappings in $\mathcal{Q} \mathcal{M}_{\text {fin }} \cup \mathcal{Q} \mathcal{M}_{\infty}$

Some subsets of $\mathcal{Q} \mathcal{M}_{\text {fin }}$ and $\mathcal{Q} \mathcal{M}_{\infty}$ have recently been studied in the literature. For instance, if $A(f)=\{\infty\}$ and $f^{-1}(A(f))$ is non-empty, then $f$ is a quasimeromorphic mapping of transcendental type with at least one pole; the Julia set for these 
mappings has been studied in Chapter 4. Further, if $S=A(f)$ is finite and is omitted in the range of $f$, then $f$ is a quasiregular mapping of $S$-transcendental type whose dynamics have been studied by Nicks and Sixsmith in [67]. We shall denote the subsets containing these mappings as $\mathcal{Q M T}$ and $\mathcal{T}_{S}$ respectively. Thus $\mathcal{T}_{S} \subset \mathcal{Q} \mathcal{M}_{\text {fin }}$ and $\mathcal{Q M T} \subset \mathcal{Q} \mathcal{M}_{\text {fin }} \cup \mathcal{Q} \mathcal{M}_{\infty}$. Further, as remarked in Section 4.1.1, any $f \in \mathcal{Q} \mathcal{M}_{\text {fin }}$ has $f^{k} \in \mathcal{T}_{S}$ for some $k \in \mathbb{N}$.

\subsection{Properties of mappings in $\mathbb{S}_{q m}$}

Recall Theorem 1.2.6, which tells us that when the mapping $f$ is quasimeromorphic near an isolated essential singularity at infinity, then every point with at most $q$ exceptions will have infinitely many preimages. It turns out that the natural analogue to this result holds for $f \in \mathbb{S}_{q m}$ by Okuyama and Pankka in [72]. We shall provide a proof here for completion. In what follows, recall for $y \in \hat{\mathbb{R}}^{d}$ the Möbius mappings $\hat{M}_{y}$ defined using equation (1.2.6).

Theorem 6.2.1. Let $d \geq 2, K>1$ and let $q=q(d, K)$ be Rickman's constant. Then if $R>0$ and $f: B_{\chi}(e, R) \backslash\{e\} \rightarrow \hat{\mathbb{R}}^{d} \backslash\left\{a_{1}, a_{2}, \ldots, a_{q}\right\}$ is a $K$ quasimeromorphic map with the $a_{i} \in \hat{\mathbb{R}}^{d}$ distinct for $i=1,2, \ldots, q$, then $f$ has a limit at $e$.

Moreover if $f \in \mathbb{S}_{q m}$ is a $K$-quasimeromorphic map and $e \in A(f)$, then given any set $X \subset \hat{\mathbb{R}}^{d}$ with $\operatorname{card}(X) \geq q$, there exists some $x \in X$ such that $f^{-1}(x)$ is infinite and contains points that are arbitrarily close to $e$.

Proof. Let $f: B_{\chi}(e, R) \backslash\{e\} \rightarrow \hat{\mathbb{R}}^{d} \backslash\left\{a_{1}, a_{2}, \ldots, a_{q}\right\}$, where $q=q(d, K)$ is Rickman's constant and the $a_{i} \in \hat{\mathbb{R}}^{d}$ are distinct. Consider the mapping $g=f \circ \hat{M}_{e}^{-1}$, which is a quasimeromorphic mapping defined on a punctured neighbourhood of infinity; this mapping will omit $\hat{M}_{e}\left(a_{i}\right)$ for all $i=1,2, \ldots, q$. Now applying the first part of Theorem 1.2.6(ii) to $g$, then $g$ has a limit at $\infty$. This corresponds to $f$ having a 
limit at $\hat{M}_{e}^{-1}(\infty)=e$.

For the second part, suppose that $f \in \mathbb{S}_{q m}$ and $e \in A(f)$. If $e$ is isolated, then the result follows by applying the second part of Theorem 1.2.6(ii) to $h=f \circ \hat{M}_{e}^{-1}$, where $f$ is restricted to the ball $B_{\chi}\left(e, R^{\prime}\right)$ and $R^{\prime}>0$ is such that $B_{\chi}\left(e, R^{\prime}\right) \cap A(f)=\{e\}$. If $e$ is not isolated, then it is the limit of isolated essential singularities $e_{n} \in A(f)$. Let $R_{n} \rightarrow 0$ be a sequence of positive real numbers so that $B_{\chi}\left(e_{n}, R_{n}\right) \cap A(f)=\left\{e_{n}\right\}$ for all $n \in \mathbb{N}$. Now for each $n \in \mathbb{N}$, by applying the argument above to $f$ restricted to $B_{\chi}\left(e_{n}, R_{n}\right)$, there exists some $x_{n} \in X$ such that $f^{-1}\left(x_{n}\right)$ is infinite and contains points arbitrarily close to $e_{n}$. As $X$ is a finite set of points, the result then follows by taking a suitable subsequence of $\left(x_{n}\right)$ to get a point $x \in X$ with an infinite sequence of points in $f^{-1}(x)$ tending to $e$.

By following the terminology of Dominguez, Montes de Oca and Sienra in [25], if $x \in \hat{\mathbb{R}}^{d}$ has finitely many preimages under a $\mathbb{S}_{q m}$ mapping $f$ in a neighbourhood of a point $e \in A(f)$, then we say that $x$ is a local Picard exceptional point with respect to $e$. We shall denote the set of such points by $P(f ; e)$. We further say that $x$ is a Picard exceptional point if $f^{-1}(x)$ is finite, and $x$ is a Fatou exceptional point if $\mathcal{O}_{f}^{-}(x)$ is finite. We shall denote the set of Fatou exceptional points by $E(f)$. From this we can observe that if $A(f)=\{\infty\}$, then $\bigcap_{k=1}^{\infty} P\left(f^{k} ; \infty\right)=E(f)$. It is clear from these definitions that all Fatou exceptional points are Picard exceptional points, whilst it follows from the above theorem that a point $x$ is a Picard exceptional point if and only if $x \in \bigcap_{e \in A(f)} P(f ; e)$. However, it is noted in $[25]$ that there exist examples in the Bolsch class $\mathbb{S}$ where local Picard exceptional points are not Picard exceptional and Picard exceptional points are not Fatou exceptional, hence these inclusions can be proper.

Using the above notation, Theorem 6.2.1 now tells us that for each $e \in A(f)$, there exist at most $q$ local Picard exceptional points. As the constant $q$ is uniform across all essential singularities of $f$, then it follows that there are at most $q$ Picard 
exceptional points and $q$ Fatou exceptional points for $f$ as well.

With Theorem 6.2.1 established, it is now possible to naturally extend Theorem 1.5.2 and its proof to the new setting and show that $\mathbb{S}_{q m}$ is indeed a class that is closed under composition. This takes the form of the following theorem.

Theorem 6.2.2. Let $d \geq 2$ be fixed and let $f, g \in \mathbb{S}_{q m}$. Then the following hold.

(i) If $U_{e}$ is any open neighbourhood of a point $e \in A(f)$, then $f$ assumes in $U_{e} \backslash A(f)$ every value in $\hat{\mathbb{R}}^{d}$ infinitely often with at most $q-1$ exceptions.

(ii) $f \circ g \in \mathbb{S}_{q m}$, with $A(f \circ g)=A(g) \cup g^{-1}(A(f))$.

Let $D \subset \hat{\mathbb{R}}^{d}$ be a domain, let $w \in \partial D$ and let $f: D \rightarrow \hat{\mathbb{R}}^{d}$ be a quasimeromorphic mapping. We say that a point $y \in \hat{\mathbb{R}}^{d}$ is an asymptotic value of $f$ at $w$ if there exists some path $\gamma:[0,1) \rightarrow D$ such that $\gamma(t) \rightarrow w$ and $f(\gamma(t)) \rightarrow y$ as $t \rightarrow 1$. For transcendental meromorphic functions on the complex plane, Iversen's Theorem states that every Picard exceptional point is also an asymptotic value at $\infty$. This result has a natural analogue in the quasimeromorphic setting, which was proven in [60]; see also [87, VII.2.6].

Theorem 6.2.3. Let $D \subset \hat{\mathbb{R}}^{d}$ be a domain, let $f: D \rightarrow \hat{\mathbb{R}}^{d}$ be a quasimeromorphic mapping and let $w \in \partial D$ be an isolated essential singularity of $f$. Then every $x \in \hat{\mathbb{R}}^{d} \backslash f(D)$ is an asymptotic value of $f$ at $w$.

Due to the local nature of the above theorem, we may apply it to each isolated essential singularity $e \in A(f)$, concluding that every point in $P(f ; e)$ is an asymptotic value of $f$ at $e$.

A final useful observation is the following. If $e \in A\left(f^{N}\right) \backslash A\left(f^{N-1}\right)$ for some $N \in \mathbb{N}$, then $f^{k}(e)$ is well-defined for $k=0,1,2, \ldots, N-1$ and $f^{k}(e) \in A\left(f^{N-k}\right)$. From the earlier discussion, this means that $P\left(f^{N-k} ; f^{k}(e)\right)$ will be finite for each $k=0,1,2, \ldots, N-1$, and consequently $\bigcup_{k=0}^{N-1} P\left(f^{N-k} ; f^{k}(e)\right)$ is also finite. 


\subsection{Julia set properties for $\mathbb{S}_{q m}$ mappings}

Following Definition 4.1.1, we shall extend the definition of the Julia set to include $f \in \mathcal{Q} \mathcal{M}_{f i n} \cup \mathcal{Q} \mathcal{M}_{\infty}$.

Definition 6.3.1. Let $d \geq 2$ and let $f: \hat{\mathbb{R}}^{d} \backslash A(f) \rightarrow \hat{\mathbb{R}}^{d}$ be a mapping from $\mathcal{Q} \mathcal{M}_{\text {fin }} \cup \mathcal{Q} \mathcal{M}_{\infty}$. Then the Julia set of $f$ is defined by

$\mathcal{J}(f):=\left\{x \in \hat{\mathbb{R}}^{d}: \operatorname{card}\left(\hat{\mathbb{R}}^{d} \backslash \mathcal{O}_{f}^{+}\left(U_{x}\right)\right)<\infty\right.$ for all neighbourhoods $U_{x} \subset \hat{\mathbb{R}}^{d}$ of $\left.x\right\}$

When $d=2$ and we identify $\mathbb{C}$ with $\mathbb{R}^{2}$ in the usual way, it is clear that the definition above agrees with the Julia set definition given for the corresponding mappings in the Bolsch class $\mathbb{S}$. Furthermore, Definition 6.3.1 agrees with the Julia set definitions given for mappings in $\mathcal{T}_{S}$ and $\mathcal{Q M T}$ respectively: for mappings in $\mathcal{T}_{S}$, the set given in equation (6.3.1) is the closure of the Julia set in $\hat{\mathbb{R}}^{d}$ rather than $\mathbb{R}^{d} \backslash S$, whilst for mappings $f \in \mathcal{Q M T}$ we have $\mathcal{J}(f)=\overline{\mathcal{O}_{f}^{-}(\infty)}=\overline{\mathcal{O}_{f}^{-}(A(f))}$ when the backward orbit of infinity is infinite. In fact, it follows from Theorem 6.2.1 that $\mathcal{J}(f)=\overline{\mathcal{O}_{f}^{-}(A(f))}$ for $f \in \mathcal{Q} \mathcal{M}_{\infty}$.

Using Definition 6.3.1 and considering the methods used in Chapter 4, we can show that $\mathcal{J}(f)$ has many of the usual properties of the classical Julia set; these are summarised below.

Theorem 6.3.2. Let $f \in \mathcal{Q M}_{\text {fin }} \cup \mathcal{Q} \mathcal{M}_{\infty}$. Then $\mathcal{J}(f)$ has the following properties:

(i) $\mathcal{J}(f)$ is closed, infinite and does not contain any isolated points;

(ii) $x \in \mathcal{J}(f) \backslash A(f)$ if and only if $f(x) \in \mathcal{J}(f)$;

(iii) $\mathcal{J}(f) \subset \overline{\mathcal{O}_{f}^{-}(x)}$ for every $x \in \hat{\mathbb{R}}^{d} \backslash E(f)$;

(iv) $\mathcal{J}(f)=\overline{\mathcal{O}_{f}^{-}(x)}$ for every $x \in \mathcal{J}(f) \backslash E(f)$; 
(v) if $U \subset \hat{\mathbb{R}}^{d}$ is an open set such that $U \cap \mathcal{J}(f) \neq \varnothing$, then $\hat{\mathbb{R}}^{d} \backslash E(f) \subset \mathcal{O}_{f}^{+}(U)$; and

(vi) for all $n \in \mathbb{N}, \mathcal{J}(f)=\mathcal{J}\left(f^{n}\right)$.

Proof. We split this proof into two cases based on the cardinality of $\mathcal{O}_{f}^{-}(A(f))$. Firstly, the case when $\mathcal{O}_{f}^{-}(A(f))$ is finite is analogous to that found in Section 4.2 by setting $S=\mathcal{O}_{f}^{-}(A(f))$.

For the case when $\mathcal{O}_{f}^{-}(A(f))$ is infinite, we first make an observation. Indeed, if $A(f)$ is finite whilst $\mathcal{O}_{f}^{-}(A(f))$ is infinite, then there exists some isolated essential singularity $e \in A(f)$ such that $\mathcal{O}_{f}^{-}(e)$ is infinite. On the other hand, if $A(f)$ is infinite then there must be infinitely many isolated essential singularities in $A(f)$. It then follows from Theorem 6.2.1 that there exists some isolated essential singularity $e \in A(f)$ such that $\mathcal{O}_{f}^{-}(e)$ is infinite.

Let $e \in A(f)$ be an isolated essential singularity with $\mathcal{O}_{f}^{-}(e)$ infinite, and recall that $\mathcal{J}(f)=\overline{\mathcal{O}_{f}^{-}(A(f))}$. We claim that $\mathcal{J}(f)=\overline{\mathcal{O}_{f}^{-}(e)}$ which shall follow by a proof similar to that of Lemma 4.3.2. First note that $\overline{\mathcal{O}_{f}^{-}(e)} \subset \overline{\mathcal{O}_{f}^{-}(A(f))}$ is obvious. Further note that it suffices to show that $\mathcal{O}_{f}^{-}(A(f)) \subset \overline{\mathcal{O}_{f}^{-}(e)}$, as $\overline{\mathcal{O}_{f}^{-}(e)}$ is closed. Let $x \in \mathcal{O}_{f}^{-}(A(f))$ and let $U_{x}$ be a neighbourhood of $x$. Then there exists some $e^{\prime} \in A(f)$ and some minimal natural $N \geq 0$ such that $f^{N}(x)=e^{\prime}$. If $N \in \mathbb{N}$, then $f^{N}: \hat{\mathbb{R}}^{d} \backslash A\left(f^{N}\right) \rightarrow \hat{\mathbb{R}}^{d}$ is a quasimeromorphic mapping, so in particular continuous and open. It follows that there exists an open set $V_{x} \subset U_{x}$ containing $x$ such that $f^{N}$ is quasimeromorphic on $V_{x}$ and $f^{N}\left(V_{x}\right)$ is a well-defined open neighbourhood of $e^{\prime}$. If $N=0$, then $f^{0}=\mathrm{id}$ and $V_{x}=V_{e^{\prime}}$ is an open neighbourhood of $e^{\prime}$.

As $\mathcal{O}_{f}^{-}(e)$ is infinite and $P\left(f ; e^{\prime}\right)$ is finite, then there exist $w_{n} \in \mathcal{O}_{f}^{-}(e)$ such that $w_{n} \rightarrow e^{\prime}$ as $n \rightarrow \infty$ and $\mathcal{O}_{f}^{-}\left(w_{n}\right)$ is infinite for all $n \in \mathbb{N}$. Now there exists some $M \in \mathbb{N}$ such that $w_{n} \in f^{N}\left(V_{x}\right)$ for all $n \geq M$. In particular, this means there exists $y_{M} \in V_{x} \subset U_{x}$ such that $f^{N}\left(y_{M}\right)=w_{M}$. As $y_{M} \in \mathcal{O}_{f}^{-}\left(w_{M}\right) \subset \mathcal{O}_{f}^{-}(e)$, then 
$U_{x} \cap \mathcal{O}_{f}^{-}(e) \neq \varnothing$. Since $U_{x}$ was an arbitrary open neighbourhood, the claim follows. The remainder of the proof of Theorem 6.3.2 now follows analogously to the proof in Section 4.3, where $\infty$ is replaced with $e$.

\subsection{Escaping set of an essential singularity}

In [4], Baker et al. studied the escaping sets of functions meromorphic outside a small set, inspired by results for the escaping sets defined for holomorphic functions in $\mathbb{C}^{*}=\mathbb{C} \backslash\{0\}$, where some points also escaped to 0 . It was shown that such sets share many of the basic properties of the normal escaping set for transcendental meromorphic mappings.

Since a function in $\mathbb{S}_{q m}$ can have more than one essential singularity, then we can consider the set of points that escape to each essential singularity under iteration. Following [25] and [4], for $f \in \mathbb{S}_{q m}$ and $e \in A(f)$, we define the escaping set to $e$ as

$$
I_{e}(f)=\left\{x \in \hat{\mathbb{R}}^{d}: f^{n}(x) \text { is defined for all } n \in \mathbb{N}, f^{n}(x) \rightarrow e \text { as } n \rightarrow \infty\right\} .
$$

As a remark, using this new notation the escaping set $I(f)$ of a quasimeromorphic mapping $f: \mathbb{R}^{d} \rightarrow \hat{\mathbb{R}}^{d}$ studied in Chapter 5 is denoted by $I_{\infty}(f)$.

Analogous to the escaping sets defined in [4] and in Chapter 5, we get that these escaping sets have similar properties. For instance, it is clear by definition that $I_{e}(f)$ is completely invariant for every $e \in A(f)$.

Theorem 6.4.1. Let $f \in \mathbb{S}_{q m}$ with $A(f) \neq \varnothing$ and let $e \in A(f)$. Then

(i) $I_{e}(f) \cap \mathcal{J}(f)$ is infinite, and

(ii) $\mathcal{J}(f) \subset \partial I_{e}(f)$.

If we assume that Theorem 6.4.1(i) holds, then (ii) follows using a small extension 
to the argument given in the proof of Theorem 5.1.2(ii). Indeed, if $\operatorname{card}(A(f))=1$, then using a suitable normalisation we get that $A(f)=\{\infty\}$ and the result holds immediately by Theorem 5.1.2(ii). If $\operatorname{card}(A(f)) \geq 2$, then the inclusion follows from the natural analogue of Lemma 5.5.1 applied to the pairwise disjoint sets $I_{e}(f)$ with $e \in A(f)$.

Similar to the case with quasiregular and quasimeromorphic mappings of transcendental type, strict inclusion can also occur in Theorem 6.4.1(ii).

Example 6.4.2. Let $f$ be the map constructed in Example 5.5.2 and consider $G:=\hat{M}_{e} \circ f^{2} \circ \hat{M}_{e}^{-1} \in \mathbb{S}_{q m}$, with $e \in A\left(f^{2}\right)=\{-1, \infty\}$. Now $f$ has a single pole and a fixed point $w \in \partial I(f) \backslash \mathcal{J}(f)$. It follows that $w \in \partial I_{\infty}\left(f^{2}\right) \backslash \mathcal{J}\left(f^{2}\right)$ and therefore $M_{e}(w) \in \partial I_{e}(G) \backslash \mathcal{J}(G)$.

To show that Theorem 6.4.1(i) does hold, we shall instead show that by suitably modifying the arguments found in Chapter 5, we can extend Theorem 5.1.1 to the new setting in the form of Theorem 6.4.6. This will state that for all functions $f \in \mathbb{S}_{q m}$ with $A(f) \neq \varnothing$, and for every $e \in A(f)$, there exists a point in the Julia set that escapes arbitrarily slowly to $e$. Theorem 6.4.1(i) will then follow from the complete invariance of $I_{e}(f)$.

First, we shall introduce some notation by Nicks and Sixsmith in [70]. First for each $y \in \hat{\mathbb{R}}^{d}$, we define the generalised modulus function with respect to $y$ on $\hat{\mathbb{R}}^{d}$ as

$$
|x|_{y}:= \begin{cases}|x| & \text { if } y=\infty \\ \frac{1}{|x-y|} & \text { otherwise. }\end{cases}
$$

Now recall the definition of $M_{y}$ from equation (1.2.5). It then follows that

$$
|x|_{y}=\left|M_{y}(x)\right| \text { for all } x, y \in \hat{\mathbb{R}}^{d} \text {. }
$$

Let $e \in A(f)$ be an isolated essential singularity and let $y \in P(f ; e)$. Then there 
exists some constant $R_{e}>0$ such that $f^{-1}(y) \cap B_{\chi}(e, r)=\varnothing$ whenever $r \leq 1 / R_{e}$. Then we can define the generalised maximum modulus from $e$ as

$$
M_{e, y}(r, f)=\max \left\{|f(x)|_{y}:|x|_{e}=r\right\}
$$

where $r>R_{e}$. Note that by Theorem 6.2.3, $y$ is an asymptotic value at $e$. As $f$ is open and $e$ is an essential singularity then, together with the maximum principle, this means that $M_{e, y}(r, f)$ is a strictly increasing function for sufficiently large $r>\rho_{e} \geq R_{e}$. For the rest of this chapter, we shall use the notation $\rho_{e}$ to denote such a constant where it exists.

Using this generalised maximum modulus, we can get an analogue of Theorem 3.1.2 and Corollary 3.1.3.

Theorem 6.4.3. Let $f \in \mathbb{S}_{q m}$ with $A(f) \neq \varnothing$, let $e \in A(f)$ be an isolated essential singularity, let $y \in P(f ; e)$ and let $\lambda>1$. Then

$$
\lim _{r \rightarrow \infty} \frac{M_{e, y}(\lambda r, f)}{M_{e, y}(r, f)}=\infty
$$

In particular,

$$
\lim _{r \rightarrow \infty} \frac{\log \left(M_{e, y}(r, f)\right)}{\log (r)}=\infty
$$

Proof. Let $M_{e}$ and $M_{y}$ be sense-preserving Möbius maps defined using equation (1.2.5). Let $g:=M_{y} \circ f \circ M_{e}^{-1}$, which is a quasiregular mapping defined on a punctured neighbourhood of infinity with an essential singularity at infinity. The result then follows from equation (6.4.2), Theorem 3.1.2 and Corollary 3.1.3.

In addition to this, there is a natural analogue of Lemma 5.1.3 and, hence, natural analogues of the 'hold-up' conditions Lemma 5.1.4 and Lemma 5.3.1 in the $\mathbb{S}_{q m}$ setting, whose proofs and statements are almost exactly the same. These are summarised in the following two lemmas. 
Lemma 6.4.4. Let $f: \hat{\mathbb{R}}^{d} \backslash A(f) \rightarrow \hat{\mathbb{R}}^{d}$ be a function, where $A(f)$ is a closed, countable set. For $n \geq 0$, let $\left(F_{n}\right)$ be a sequence of non-empty sets compactly contained in $\hat{\mathbb{R}}^{d} \backslash A(f),\left(\ell_{n+1}\right)$ be a sequence of natural numbers and $G_{n} \subset F_{n}$ be a sequence of non-empty subsets such that $f^{\ell_{n+1}}$ is continuous on $\overline{G_{n}}$ with

$$
f^{\ell_{n+1}}\left(G_{n}\right) \supset F_{n+1}
$$

For $n \in \mathbb{N}$, set $r_{n}=\sum_{i=1}^{n} \ell_{i}$. Then there exists $\zeta \in \overline{F_{0}}$ such that $f^{r_{n}}(\zeta) \in \overline{F_{n}}$ for each $n \in \mathbb{N}$.

Further, suppose that $f \in \mathcal{Q M}_{\text {fin }} \cup \mathcal{Q} \mathcal{M}_{\infty}$ is such that for $n \geq 0, f^{\ell_{n+1}}$ is quasimeromorphic on $\overline{G_{n}}$ and equation (6.4.4) holds. If there is a subsequence $\left(F_{n_{k}}\right)$ such that $\overline{F_{n_{k}}} \cap \mathcal{J}(f) \neq \varnothing$ for all $k \in \mathbb{N}$, then $\zeta$ can be chosen to be in $\mathcal{J}(f) \cap \overline{F_{0}}$.

Lemma 6.4.5. Let $f \in \mathcal{Q} \mathcal{M}_{\text {fin }} \cup \mathcal{Q} \mathcal{M}_{\infty}$ and let $e \in A(f)$. Let $p \in \mathbb{N}$ and for $m \in \mathbb{N}$ and $i \in\{1,2, \ldots, p\}$, let $X_{m}^{(i)}$ be non-empty sets compactly contained in $\hat{\mathbb{R}}^{d} \backslash A(f)$, with $X_{m}=\bigcup_{i=1}^{p} X_{m}^{(i)}$ such that

$$
\inf \left\{|x|_{e}: x \in X_{m}\right\} \rightarrow \infty \text { as } m \rightarrow \infty
$$

Suppose that there exists a strictly increasing sequence of integers $\left(m_{t}\right)$ such that either (X1)-(X3) from Lemma 5.1.4 hold, or (X4)-(X6) from Lemma 5.3 .1 hold. Then given any positive sequence $a_{n} \rightarrow \infty$, there exists $\zeta_{e} \in I_{e}(f) \cap \mathcal{J}(f)$ and $N \in \mathbb{N}$ such that $\left|f^{n}\left(\zeta_{e}\right)\right|_{e} \leq a_{n}$ whenever $n \geq N$.

Theorem 6.4.6. Let $f \in \mathbb{S}_{q m}$ with $A(f) \neq \varnothing$ and let $e \in A(f)$. Then for any positive sequence $a_{n} \rightarrow \infty$, there exists $\zeta_{e} \in I_{e}(f) \cap \mathcal{J}(f)$ and $N \in \mathbb{N}$ such that $\left|f^{n}\left(\zeta_{e}\right)\right|_{e} \leq a_{n}$ whenever $n \geq N$.

Proof. Let $a_{n} \rightarrow \infty$ be a given positive sequence. Firstly, suppose that $e \in A(f)$ is isolated and let $g:=\hat{M}_{e} \circ f \circ \hat{M}_{e}^{-1}$. Let $U_{e}$ be a neighbourhood of $e$ such that 
$U_{e} \cap A(f)=\{e\}$. If $f^{-1}(e) \cap U_{e}=\varnothing$, then $g$ will be a quasiregular mapping defined on $\hat{M}_{e}\left(U_{e} \backslash\{e\}\right)$, which is a punctured neighbourhood of the essential singularity at infinity. It follows that for sufficiently large $m \in \mathbb{N}$, the sets $X_{m}$ found in the proof of Theorem 5.1.1 in Section 5.2 satisfy equation (5.1.2) and (X1)-(X3) under $g$. Now the sets $\hat{M}_{e}\left(X_{m}\right)$ satisfy equation (6.4.5) and (X1)-(X3) under $f$. Therefore an application of Lemma 6.4.5 yields a point $\zeta_{e} \in I_{e}(f) \cap \mathcal{J}(f)$ and some $N \in \mathbb{N}$ such that $\left|f^{n}\left(\zeta_{e}\right)\right|_{e} \leq a_{n}$ whenever $n \geq N$.

Next, suppose that $f^{-1}(e) \cap U_{e} \neq \varnothing$. If $f^{-1}(e) \cap U_{e}$ is finite, then we can shrink $U_{e}$ and apply the above argument to get the result. Now suppose that $f^{-1}(e) \cap U_{e}$ is infinite. Then $g$ will be a quasimeromorphic mapping defined on a punctured neighbourhood of infinity, with infinitely many poles and an essential singularity at infinity. It follows that for sufficiently large $m \in \mathbb{N}$, the sets $X_{m}$ found in the proof of Theorem 5.1.1 in Section 5.3 satisfy equation (5.1.2) and (X4)-(X6) under $g$. As before, this means that $\hat{M}_{e}\left(X_{m}\right)$ satisfy equation (6.4.5) and (X4)-(X6) under $f$, so an application of Lemma 6.4.5 gives the result.

Finally suppose that $e \in A(f)$ is not isolated, so there exist isolated essential singularities $e_{k} \in A(f)$ with $e_{k} \rightarrow e$ as $k \rightarrow \infty$. Using Theorem 6.2.1 and noting that $P\left(f ; e_{k}\right)$ is finite for each $k$, then by taking a subsequence and relabelling we may assume without loss of generality that $e_{k+1} \notin P\left(f ; e_{k}\right)$ and $e_{k} \in B_{\chi}(e, 1 / k)$ for all $k \in \mathbb{N}$. Now for each $k, \ell \in \mathbb{N}$, let $x_{k, \ell} \in f^{-1}\left(e_{k+1}\right)$ be such that $x_{k, \ell} \rightarrow e_{k}$ as $\ell \rightarrow \infty$. By removing finitely many terms and relabelling, we may also assume that $x_{k, \ell} \notin P\left(f ; e_{k+1}\right)$ and $x_{k, \ell} \in B_{\chi}(e, 1 / k)$ for each $k, \ell \in \mathbb{N}$.

Now considering a generalised version of Lemma 4.4 .2 for mappings in $\mathcal{Q} \mathcal{M}_{\infty}$ with isolated essential singularities, we shall construct sequences $x_{m},\left(U_{m}\right)$ and $\left(E_{m}\right)$ as follows.

Set $x_{1}=x_{1,1}$ and $U_{1}$ to be an open neighbourhood of $x_{1}$ such that $\bar{U}_{1} \cap A(f)=\varnothing$, $\bar{U}_{1} \subset B_{\chi}(e, 1)$ and $\bar{U}_{1} \cap P\left(f ; e_{2}\right)=\varnothing$. Now suppose that $x_{m-1}$ and $U_{m-1}$ have 
been chosen for some $m \geq 2$, so $f\left(U_{m-1}\right)$ is an open neighbourhood of $e_{m}$. Then there exists some $\ell \in \mathbb{N}$ such that $x_{m, \ell} \in B_{\chi}(e, 1 / m) \cap f\left(U_{m-1}\right)$. Set $x_{m}=x_{m, \ell}$ and set $U_{m} \subset f\left(U_{m-1}\right)$ to be a neighbourhood of $x_{m}$ such that $\bar{U}_{m} \cap A(f)=\varnothing$, $\bar{U}_{m} \subset B_{\chi}(e, 1 / m)$ and $\bar{U}_{m} \cap P\left(f ; e_{m+1}\right)=\varnothing$.

Finally, for each $m \in \mathbb{N}$, by applying a generalised version of Lemma 4.4.2, choose a non-empty open region $E_{m}$ such that $\bar{E}_{m} \cap A(f)=\varnothing, \bar{E}_{m} \subset B_{\chi}(e, 1 / m)$ and

$$
f\left(U_{m}\right) \supset \bar{E}_{m} \text { and } f\left(E_{m}\right) \supset \bar{U}_{m}
$$

With the $x_{m}, U_{m}$ and $E_{m}$ established, for each $m \in \mathbb{N}$ define $X_{m}^{(1)}=U_{m}$ and $X_{m}^{(2)}=E_{m}$. Now observe that $\inf \left\{|x|_{e}: x \in U_{m} \cup E_{m}\right\} \geq m$ for all $m \in \mathbb{N}$, thus equation (6.4.5) is satisfied. Further, (X4)-(X6) are all satisfied using a similar argument to that in the proof of Theorem 5.1.1 in Section 5.3, using $p=2$, the subsequence $m_{t}=t$ for $t \in \mathbb{N}$, and Theorem 6.3.2(ii). Hence the proof follows by Lemma 6.4.5.

\subsection{The generalised escaping set}

Recall from Chapter 4 the definition of the bungee set $B U(f)$ for a quasimeromorphic mapping $f$. There, every element $x \in B U(f)$ is such that the sequence of iterates $f^{n}(x)$ contains a subsequence that tends to infinity and another subsequence that is bounded away from infinity. In the Bolsch class, it was shown in [25] that it is possible for there to exist a mapping $g \in \mathbb{S}$ and a point $y$ for which the sequence of iterates $g^{n}(y)$ contain subsequences that only tend to different essential singularities in $A(g)$. In some sense, this point can be viewed as both a 'bungee point' and an escaping point.

Let $f \in \mathbb{S}_{q m}$ and let $x \in \hat{\mathbb{R}}^{d} \backslash \mathcal{O}_{f}^{-}(A(f))$. Then we define the omega limit set for $x$ 
under $f$ as

$\omega(x, f):=\left\{y \in \hat{\mathbb{R}}^{d}: f^{n_{k}}(x) \rightarrow y\right.$ for some sequence of natural numbers $\left.n_{k} \rightarrow \infty\right\}$.

Informally, the omega limit set for $x$ is the collection of possible limit points of all subsequences of iterates of a given point $x$ under $f$. As a remark, if $f$ is a quasiregular or quasimeromorphic mapping of transcendental type, whereby $A(f)=\{\infty\}$, then $x \in B U(f)$ if and only if $\operatorname{card}(\omega(x, f))>1$ and $\infty \in \omega(x, f)$.

Now following [25], we define the generalised escaping set $I_{g}(f)$ as

$$
I_{g}(f):=\left\{x \in \hat{\mathbb{R}}^{d}: \omega(x, f) \subset \mathcal{O}_{f}^{-}(A(f))\right\}
$$

Immediately by the definition of $I_{e}(f)$ and Theorem 6.4.1, we get some basic properties akin to the classical properties of the escaping set.

Theorem 6.5.1. Let $f \in \mathbb{S}_{q m}$ with $A(f) \neq \varnothing$. Then:

(i) $\bigcup_{e \in A(f)} I_{e}(f) \subset I_{g}(f)$, and

(ii) $I_{g}(f) \cap \mathcal{J}(f)$ is infinite.

For the Bolsch class $\mathbb{S}$, there are many open questions in [25] regarding the possible behaviour of points in $I_{g}(f)$. For instance,

Question 6.13 Does there exist any $f \in \mathbb{S}$ with $\operatorname{card}(A(f)) \geq 2$ where equality is achieved in Theorem 6.5.1(i)?

Question 6.15 If $f \in \mathbb{S}$ has at least one non-omitted singularity in $A(f)$, then can it ever be true that $\omega(x, f) \subset A(f)$ for all $x \in I_{g}(f)$ ?

Question 6.19 Is there an example of a function in $\mathbb{S}$ with a point whose omega limit set is infinite? 
Martí-Pete in [56] showed that the answer to the first question was no for all transcendental self-maps $f: \mathbb{C}^{*} \rightarrow \mathbb{C}^{*}$ with $A(f)=\{0, \infty\}$.

We will show that the answer to the first two of these questions is always no, including for mappings in the new class $\mathbb{S}_{q m}$, through the following result.

Theorem 6.5.2. Let $f \in \mathbb{S}_{q m}$ be such that $\operatorname{card}\left(\mathcal{O}_{f}^{-}(A(f))\right) \geq 2$, and let $X \subset$ $\mathcal{O}_{f}^{-}(A(f))$ be a finite, non-empty set. Then there exists a point $x \in I_{g}(f)$ such that $\omega(x, f)=\mathcal{O}_{f}^{+}(X)$.

To answer the third question, we shall show that it is sufficient for $A(f)$ to be infinite for a function $f \in \mathbb{S}_{q m}$ to have a point with an infinite omega limit set in $I_{g}(f)$.

Theorem 6.5.3. Let $f \in \mathbb{S}_{q m}$ be such that $A(f)$ is infinite. Then there exists $x \in I_{g}(f)$ such that $\omega(x, f)$ is infinite.

If $f$ is a quasimeromorphic mapping of transcendental type with $\mathcal{O}_{f}^{-}(\infty)$ infinite, then by applying the above theorem to a suitable iterate $f^{k}, k \in \mathbb{N}$ large, we get the following immediate corollary.

Corollary 6.5.4. Let $d \geq 2$ and $f: \mathbb{R}^{d} \rightarrow \hat{\mathbb{R}}^{d}$ be a quasimeromorphic mapping of transcendental type with $\mathcal{O}_{f}^{-}(\infty)$ infinite. Then there exists some $x \in B U(f)$ such that $\omega(x, f)$ is infinite.

\subsubsection{Proof of Theorem 6.5.2}

In what follows, recall that the spherical distance between two points $x, y \in \hat{\mathbb{R}}^{d}$ is denoted by $d_{\chi}(x, y)$. Further, we shall be using Lemma 6.4 .4 in the case that $\ell_{n}=1$ for all $n \in \mathbb{N}$.

Let $f \in \mathbb{S}_{q m}$ and suppose that $X=\left\{x_{1}, x_{2}, \ldots, x_{P}\right\} \subset \mathcal{O}_{f}^{-}(A(f))$ is a non-empty, 
finite set of points, with $P \in \mathbb{N}$. First, we shall introduce some notation. For each $i=1,2, \ldots, P$, let $N_{i} \in \mathbb{N} \cup\{0\}$ be the smallest integer such that $f^{N_{i}}\left(x_{i}\right) \in A(f)$. Now for all $t=0,1,2, \ldots, N_{i}$ let $x(i, t)=f^{N_{i}-t}\left(x_{i}\right)$, so that $x\left(i, N_{i}\right)=x_{i}$ and $x(i, 0) \in A(f)$. Informally, this notation identifies which $x_{i} \in X$ orbit we are considering and how many iterates $t$ are required before we reach an essential singularity of $f$.

Since $X$ is finite, then $\mathcal{O}_{f}^{+}(X)$ is also finite. It follows that for all $m \in \mathbb{N}$, all $i=1,2, \ldots, P$ and all $t=0,1,2, \ldots, N_{i}$, there exist open neighbourhoods $U(m, i, t)$ of $x(i, t)$ and punctured neighbourhoods $U^{*}(m, i, t):=U(m, i, t) \backslash\{x(i, t)\}$ such that,

(i) $\overline{U(m, i, t)} \cap A(f)=\varnothing$ if $t \geq 1$,

(ii) $U(m, i, t) \cap \mathcal{O}_{f}^{+}(X)=\{x(i, t)\}$,

(iii) $\sup \left\{d_{\chi}(y, x(i, t)): y \in U(m, i, t)\right\} \rightarrow 0$ as $m \rightarrow \infty$,

(iv) $f(U(m, i, t)) \supset U(m, i, t-1)$ if $t \geq 1$, and

(v) $U^{*}\left(m, i, N_{i}\right) \cap P(f ; x(j, 0))=\varnothing$ for all $j=1,2, \ldots, P$.

We shall now consider cases based on exceptional points and whether any of the $x(i, 0)$ are non-isolated essential singularities. Firstly, suppose that there exists some $i, j \in\{1,2, \ldots, P\}$ such that $x\left(i, N_{i}\right) \notin P(f ; x(j, 0))$. If $i \neq j$ then by reordering $X$ we may assume that $i=1$ and $j=P$. Set $P^{\prime}=P$ in this case. Otherwise, if $i=j$, then reordering $X$ we may assume that $j=1$. Then set $P^{\prime}=P+1$ and $x\left(P^{\prime}, t\right)=x(1, t)$ for all $t=0,1,2, \ldots, N_{1}$, with $N_{P^{\prime}}:=N_{1}$. Therefore in either scenario we have $\left.x\left(1, N_{1}\right)\right) \notin P\left(f ; x\left(P^{\prime}, 0\right)\right)$; this gives us the first case.

Case 1. $\left.x\left(1, N_{1}\right)\right) \notin P\left(f ; x\left(P^{\prime}, 0\right)\right)$.

Proof of Theorem 6.5.2 in Case 1. We shall define the sets $G(m, i, t)$ inductively as follows. Firstly as $x\left(1, N_{1}\right) \notin P\left(f ; x\left(P^{\prime}, 0\right)\right)$, then for each $m \in \mathbb{N}$, set $G\left(m, P^{\prime}, 0\right)$ 
to be an open set compactly contained in $U\left(m, P^{\prime}, 0\right) \backslash A(f)$ such that $f\left(G\left(m, P^{\prime}, 0\right)\right)$ is an open neighbourhood of $x\left(1, N_{1}\right)$. By taking a suitable subsequence and relabelling, we may assume without loss of generality that $f\left(G\left(m, P^{\prime}, 0\right)\right)$ covers $\overline{U\left(m+1,1, N_{1}\right)}$ for every $m \in \mathbb{N}$. Now for each fixed $m \in \mathbb{N}$, we shall choose sets $G(m, i, t)$ with $i=1,2, \ldots, P^{\prime}$ and $t=0,1,2, \ldots, N_{i}$ as follows.

If $N_{P^{\prime}} \in \mathbb{N}$, then using (iv), for each $t=0,1,2, \ldots, N_{P^{\prime}}-1$ choose $G\left(m, P^{\prime}, t+1\right)$ to be an open set compactly contained in $U^{*}\left(m, P^{\prime}, t+1\right)$ such that

$$
f\left(G\left(m, P^{\prime}, t+1\right)\right) \supset \overline{G\left(m, P^{\prime}, t\right)} .
$$

Next using (v), Theorem 6.2.1 and a compactness argument, choose an open set $G\left(m, P^{\prime}-1,0\right)$ compactly contained in $U\left(m, P^{\prime}-1,0\right) \backslash A(f)$ such that,

$$
f\left(G\left(m, P^{\prime}-1,0\right)\right) \supset \overline{G\left(m, P^{\prime}, N_{P^{\prime}}\right)}
$$

We now repeat the above method to define $G(m, i, t)$ for $i=1,2,3, \ldots, P^{\prime}-1$ and $t=0,1,2, \ldots, N_{i}$ as follows. Assume the sets $G(m, i, t)$ have been defined for some $i$, and $t$. If $t<N_{i}$, then by applying (iv), choose an open set $G(m, i, t+1)$ compactly contained in $U^{*}(m, i, t+1)$ such that

$$
f(G(m, i, t+1)) \supset \overline{G(m, i, t)} .
$$

Applying (v), Theorem 6.2.1 and a compactness argument once again, then for $i=2,3, \ldots, P^{\prime}-1$, choose $G(m, i-1,0)$ to be an open set compactly contained in $U(m, i-1,0) \backslash A(f)$ such that

$$
f(G(m, i-1,0)) \supset \overline{G\left(m, i, N_{i}\right)} .
$$




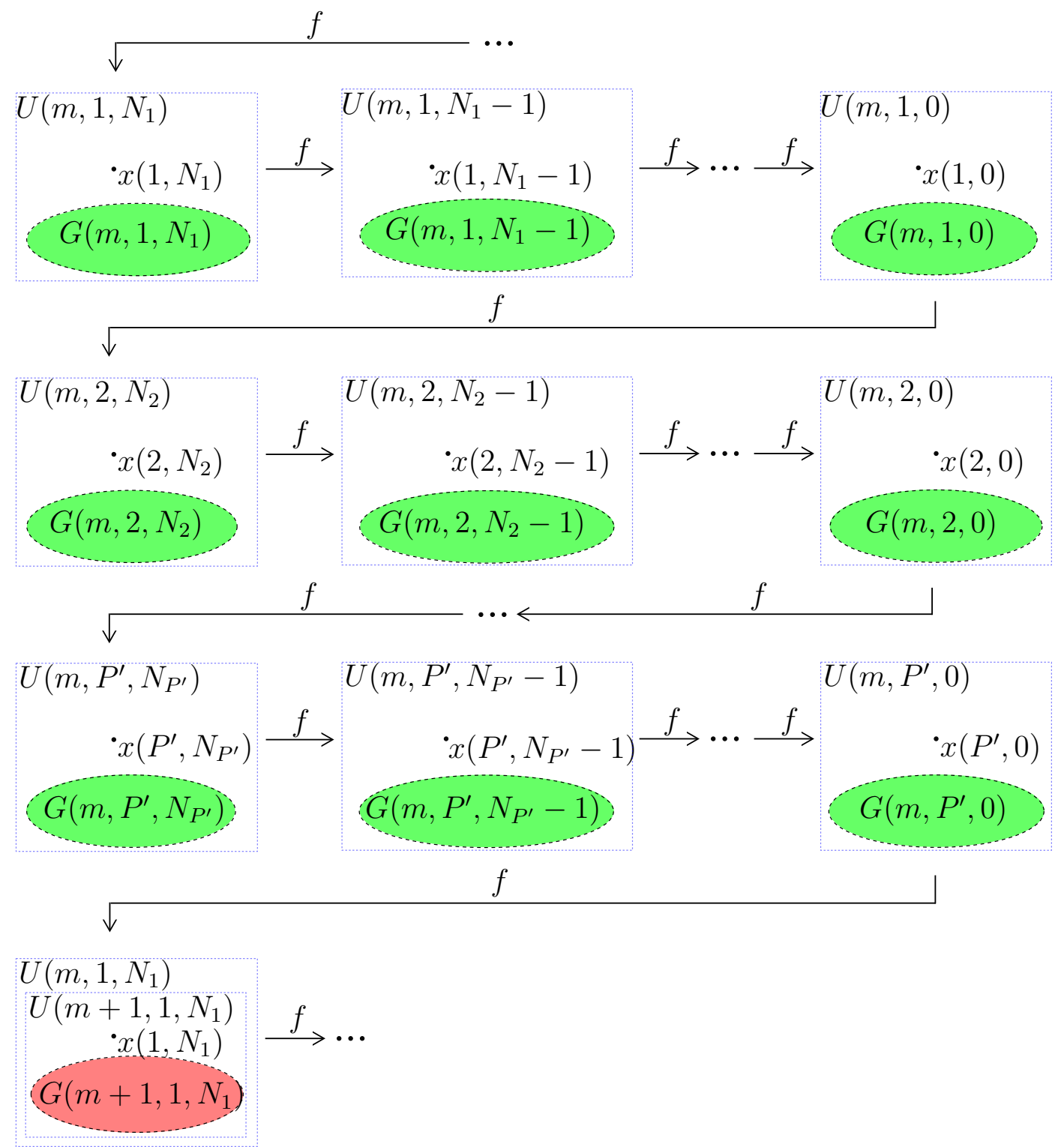

Figure 6.1: The sequence of covering sets constructed in Case 1 for each $m \in \mathbb{N}$. 
It remains to note that as $f\left(G\left(m, P^{\prime}, 0\right)\right)$ covers $\overline{U\left(m+1,1, N_{1}\right)}$, then

$$
f\left(G\left(m, P^{\prime}, 0\right)\right) \supset \overline{G\left(m+1,1, N_{1}\right)} .
$$

Finally inductively define the sets $F_{n}$, with $n \in \mathbb{N}$, as follows. First, set $F_{0}=$ $G\left(1,1, N_{1}\right)$ and then for each natural $n \geq 0$, define

$$
F_{n+1}= \begin{cases}G(m, i, t-1) & \text { if } F_{n}=G(m, i, t), t>0 \\ G\left(m, i+1, N_{i+1}\right) & \text { if } F_{n}=G(m, i, 0), i<P^{\prime} \\ G\left(m+1,1, N_{1}\right) & \text { if } F_{n}=G\left(m, P^{\prime}, 0\right) .\end{cases}
$$

In light of (iii) we can apply Lemma 6.4 .4 , with $\ell_{n}=1$ for all $n$, to the sets $F_{n}$ and $G_{n}=F_{n}$. This gives us a point $x \in I_{g}(f)$ such that $\omega(x, f)=\mathcal{O}_{f}^{+}(X)$, completing the proof of Theorem 6.5.2 in this case; see Figure 6.1.

For the second case, we now assume that for all $i, j \in\{1,2, \ldots, P\}$ we have $x\left(i, N_{i}\right) \in P(f ; x(j, 0))$ and there is at least one $x(i, 0)$ that is not an isolated essential singularity. By reordering $X$, we may assume that $x(P, 0)$ is not isolated.

Case 2. $x\left(i, N_{i}\right) \in P(f ; x(j, 0))$ for all $i, j \in\{1,2, \ldots, P\}$ and $x(P, 0)$ is a nonisolated essential singularity with respect to $f$.

Proof of Theorem 6.5.2 in Case 2. As $x(P, 0)$ is a non-isolated essential singularity then, by taking a subsequence and relabelling if necessary, for each $m \in \mathbb{N}$ there exists an isolated essential singularity $w_{m} \in A(f) \cap U(m, P, 0)$, and $w_{m} \rightarrow x(P, 0)$ as $m \rightarrow \infty$. Since each $w_{m}$ is an isolated essential singularity, it follows that there exist real numbers $\delta_{m}>0$ such that the sets $G(m, P, 0):=B_{\chi}\left(w_{m}, \delta_{m}\right)$ are compactly contained in $U^{*}(m, P, 0)$ and $\overline{G(m, P, 0)} \cap A(f)=\left\{w_{m}\right\}$.

Next if $N_{P} \in \mathbb{N}$ then, using (iv), for each $m \in \mathbb{N}$ and $t=0,1,2, \ldots, N_{P-1}$, let 
$G(m, P, t+1)$ be sets compactly contained in $U^{*}(m, P, t+1)$ such that

$$
f(G(m, P, t+1)) \supset \overline{G(m, P, t)} .
$$

Now fix some $m \in \mathbb{N}$. By (v), Theorem 6.2.1 and a compactness argument like that in the proof of Lemma 4.4.2, there must exist infinitely many open sets $G(m, P-1,0, k)$ compactly contained in $U(m, P-1,0) \backslash A(f)$ such that for all $k \neq k^{\prime}$,

$$
\overline{G(m, P-1,0, k)} \cap \overline{G\left(m, P-1,0, k^{\prime}\right)}=\varnothing,
$$

and for each $k \in \mathbb{N}$,

$$
f(G(m, P-1,0, k)) \supset \overline{G\left(m, P, N_{P}\right)} .
$$

Applying (iv) again, for each $k \in \mathbb{N}$ and $t=0,1, \ldots, N_{P-1}-1$, there exist open sets $G(m, P-1, t+1, k)$ compactly contained in $U^{*}(m, P-1, t+1)$ such that

$$
f(G(m, P-1, t+1, k)) \supset \overline{G(m, P-1, t, k)} .
$$

We now inductively define $G(m, i, t, k)$ for $i=1,2, \ldots, P-2$, for $t=0,1,2, \ldots, N_{i}$ and for each $k \in \mathbb{N}$ using the same method above as follows. Assume for each $k$ the sets $G(m, i, t, k)$ have been defined for some $i$ and $t$. If $t<N_{i}$, then using (iv), choose open sets $G(m, i, t+1, k)$ compactly contained in $U^{*}(m, i, t+1)$ such that

$$
f(G(m, i, t+1, k)) \supset \overline{G(m, i, t, k)} .
$$

Applying (v), Theorem 6.2.1 and a compactness argument, for $i=2,3, \ldots, P-2$ choose $G(m, i-1,0, k)$ to be open sets compactly contained in $U(m, i-1,0) \backslash A(f)$ such that

$$
f(G(m, i-1,0, k)) \supset \overline{G\left(m, i, N_{i}, k\right)} .
$$




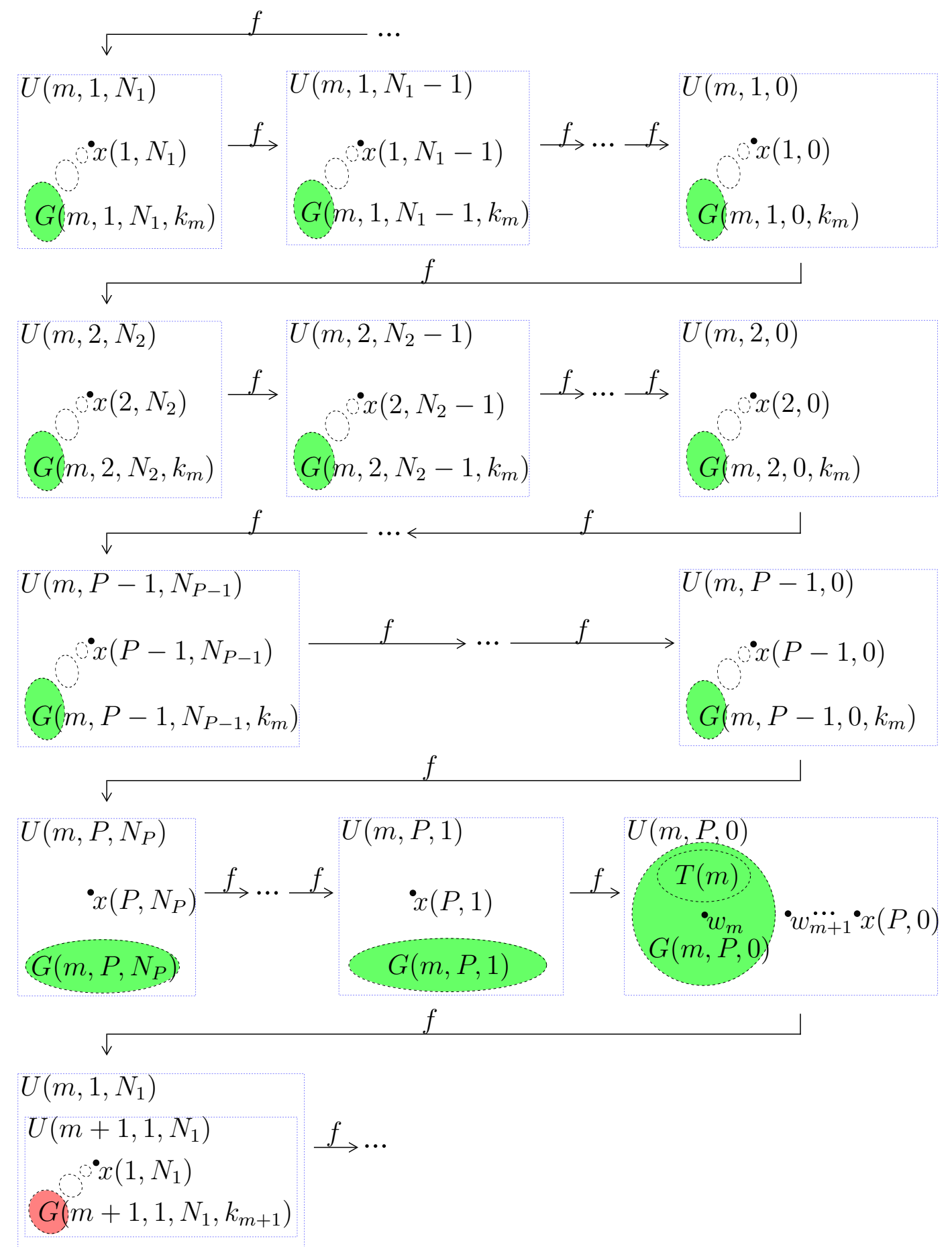

Figure 6.2: The sequence of covering sets constructed in Case 2 for each $m \in \mathbb{N}$. 
By combining equations (6.5.2) and (6.5.4)-(6.5.7), for each $k \in \mathbb{N}, i=1,2, \ldots, P$ and $t=0,1,2, \ldots, N_{i}$ we get infinitely many sequences of sets $G(m, i, t, k)$ compactly contained in $U^{*}(m, i, t) \backslash A(f)$. It remains to choose suitable $k_{m}$ for each $m \in \mathbb{N}$ to get coverings from $G(m, P, 0)$ to $\overline{G\left(m+1,1, N_{1}, k_{m+1}\right)}$.

Initialise $k_{1}=1$ and note that for all $m \in \mathbb{N}$, we have that $w_{m} \in A(f)$ implies $P\left(f ; w_{m}\right)$ is finite. Now using equation (6.5.3), we can choose some $k_{m+1} \in \mathbb{N}$ such that $\overline{G\left(m+1,1, N_{1}, k_{m+1}\right)} \cap P\left(f ; w_{m}\right)=\varnothing$. It follows by Theorem 6.2 .1 and a compactness argument that there is some open subset $T(m)$ compactly contained in $G(m, P, 0) \backslash\left\{w_{m}\right\}$ such that

$$
f(T(m)) \supset \overline{G\left(m+1,1, N_{1}, k_{m+1}\right)} .
$$

Finally we inductively define the sets $F_{n}$, with $n \in \mathbb{N}$, as follows. Firstly, set $F_{0}=G\left(1,1, N_{1}, k_{1}\right)$ and then for each natural $n \geq 0$, define

$$
F_{n+1}= \begin{cases}G\left(m, i, t-1, k_{m}\right) & \text { if } F_{n}=G\left(m, i, t, k_{m}\right), t>0 \\ G\left(m, i+1, N_{i+1}, k_{m}\right) & \text { if } F_{n}=G\left(m, i, 0, k_{m}\right), i<P-2 \\ G\left(m, P, N_{P}\right) & \text { if } F_{n}=G\left(m, P-1,0, k_{m}\right) \\ G(m, P, t-1) & \text { if } F_{n}=G(m, P, t), t>1 \\ T(m) & \text { if } F_{n}=G(m, P, 1) \\ G\left(m+1,1, N_{1}, k_{m+1}\right) & \text { if } F_{n}=T(m) .\end{cases}
$$

Finally by considering (iii), we can apply Lemma 6.4 .4 , with $\ell_{n}=1$ for all $n$, to our sets $F_{n}$ and $G_{n}=F_{n}$. This gives us a point $x \in I_{g}(f)$ such that $\omega(x, f)=\mathcal{O}_{f}^{+}(X)$, completing the proof of Theorem 6.5.2 in this case; see Figure 6.2.

For the final case, we shall require a spherical shell covering result established by 
Nicks and Sixsmith in [70]. For notation, given $y \in \hat{\mathbb{R}}^{d}$ and $r<s$, we shall define

$$
A_{y}(r, s):=\left\{x \in \hat{\mathbb{R}}^{d}: r<|x|_{y}<s\right\}
$$

where $|x|_{y}$ is defined as in equation (6.4.2). Further, recall the generalised maximum modulus defined in equation (6.4.3). The proof of this theorem is analogous to that of [70, Lemma 4.4], using Theorem 3.1.2.

Lemma 6.5.5. Let $f \in \mathbb{S}_{q m}$, let $e \in A(f)$ be an isolated essential singularity of $f$ and let $\alpha, \beta>1$. Then there exists a constant $r_{0} \geq \rho_{e}$ such that for all $y \in P(f ; e)$ and $r>r_{0}$, there exists $R>M_{e, y}(r, f)$ such that

$$
f\left(\overline{A_{e}(r, \alpha r)}\right) \supset \overline{A_{y}(R, \beta R)} .
$$

Further, recall from Section 5.3 the notation $[i]_{p}=i(\bmod p)$ for $i \in \mathbb{N}$ and some fixed $p \in \mathbb{N}$.

Case 3. For all $i, j \in\{1,2, \ldots, P\}$ we have $x\left(i, N_{i}\right) \in P(f ; x(j, 0))$, and all $x(i, 0)$ are isolated essential singularities of $f$.

Proof of Theorem 6.5.2 in Case 3. Firstly note that for each $i=1,2, \ldots, P$, we have that $x_{i}:=x\left(i, N_{i}\right) \in A\left(f^{N_{i}+1}\right)$ is an isolated essential singularity with respect to $f^{N_{i}+1}$. Further, as $x_{i} \in P(f ; x(j, 0))$ for all $i, j \in\{1,2, \ldots, P\}$, it follows that $x_{i+1} \in P\left(f^{N_{i}+1} ; x_{i}\right)$ for $i=1,2, \ldots, P-1$, and $x_{1} \in P\left(f^{N_{P}+1} ; x_{P}\right)$.

Now there exists some constant $\rho>\max \left\{\rho_{i}: i=1,2, \ldots, P\right\}$ such that the generalised maximum modulus $M_{x_{i}, x_{i+1}}\left(r, f^{N_{i}+1}\right)$ and $M_{x_{P}, x_{1}}\left(r, f^{N_{P}+1}\right)$ is well-defined for all $i=1,2, \ldots, P$ when $r>\rho$. By increasing $\rho$ if necessary, then by Corollary 6.4.3 we may assume that $M_{x_{i}, x_{i+1}}\left(r, f^{N_{i}+1}\right)>2 r$ and $M_{x_{P}, x_{1}}\left(r, f^{N_{P}+1}\right)>2 r$ whenever $r>\rho$.

For each $i=1,2, \ldots, P-1$, we shall inductively define sequences of real numbers 
$(r(m, i)) \rightarrow \infty$ as $m \rightarrow \infty$ as follows. Initialise $r(1,1)>r_{0}$ sufficiently large such that

$$
\overline{A_{x_{1}}(r(1,1), 2 r(1,1))} \subset U\left(1,1, N_{1}\right)
$$

where $r_{0} \geq \rho$ is the constant found in Lemma 6.5.5.

Now for each fixed $m \in \mathbb{N}$ and each $i=1,2, \ldots, P-1$, Lemma 6.5 .5 yields $r(m, i+1)>M_{x_{i}, x_{i+1}}\left(r(m, i), f^{N_{i}+1}\right)$ such that

$$
f^{N_{i}+1}\left(\overline{A_{x_{i}}(r(m, i), 2 r(m, i))}\right) \supset \overline{A_{x_{i+1}}(r(m, i+1), 2 r(m, i+1))},
$$

and $r(m+1,1)>M_{x_{P}, x_{1}}\left(r(m, P), f^{N_{P}+1}\right)$ such that

$$
f^{N_{P}+1}\left(\overline{A_{x_{P}}(r(m, P), 2 r(m, P))}\right) \supset \overline{A_{x_{1}}(r(m+1,1), 2 r(m+1,1))} .
$$

Note that as $f^{N_{i}-t}$ is continuous at $x_{i}=x\left(i, N_{i}\right)$ for all $i=1,2, \ldots, P$ and $t=0,1,2, \ldots, N_{i}$, then

$$
\sup \left\{d_{\chi}(y, x(i, t)): y \in f^{N_{i}-t}\left(\overline{A_{x_{i}}(r(m, i), 2 r(m, i))}\right)\right\} \rightarrow 0 \text { as } m \rightarrow \infty .
$$

Finally we inductively define the sets $F_{n}$ with $n \in \mathbb{N}$ as follows. First, initialise $F_{0}=A_{x_{1}}(r(1,1), 2 r(1,1))$ and then for each natural $n \geq 0$, define

$$
F_{n+1}= \begin{cases}A_{x_{i+1}}(r(m, i+1), 2 r(m, i+1)) & \text { if } F_{n}=A_{x_{i}}(r(m, i), 2 r(m, i)), i<P \\ A_{x_{1}}(r(m+1,1), 2 r(m+1,1)) & \text { if } F_{n}=A_{x_{P}}(r(m, P), 2 r(m, P)) .\end{cases}
$$

In light of equation (6.5.12), we can apply Lemma 6.4.4 with $\ell_{n+1}=N_{[n+1]_{P}}+1$ for each natural $n \geq 0$ to the sets $F_{n}$ and $G_{n}=F_{n}$ to get a point $x \in I_{g}(f)$ such that $\omega(x, f)=\mathcal{O}_{f}^{+}(X)$, concluding the proof of Theorem 6.5.2. 


\subsubsection{Proof of Theorem 6.5.3}

Since $A(f)$ is infinite, then there will exist some non-isolated essential singularity $e_{0} \in A(f)$ and some sequence of isolated essential singularities $e_{n} \in A(f)$ such that $e_{n} \rightarrow e_{0}$ as $n \rightarrow \infty$. For $m \in \mathbb{N}$, let $(\sigma(m))$ be the sequence $(1,2,1,2,3,1,2,3,4, \ldots)$, formed by concatenating sequences of consecutive numbers of length $n \geq 2$. This will dictate the order in which we cover sets contained in the neighbourhoods of $e_{n}$.

For $m \in \mathbb{N}$, let $U_{0}(m)$ be open neighbourhoods of $e_{0}$ such that

$$
\sup \left\{d_{\chi}\left(y, e_{0}\right): y \in U_{0}(m)\right\} \rightarrow 0 \text { as } m \rightarrow \infty
$$

As $P\left(f ; e_{0}\right)$ is finite, then we can assume that $e_{n} \notin P\left(f ; e_{0}\right)$ for all $n \in \mathbb{N}$. Now for each $m \in \mathbb{N}$, there exist $w(m, k) \in U_{0}(m) \backslash A(f)$ such that $f(w(m, k))=e_{\sigma(m)}$ for all $k \in \mathbb{N}$. For each $m \in \mathbb{N}$, it follows that there exist open neighbourhoods $G(m, k)$ of $w(m, k)$, compactly contained in $U_{0}(m) \backslash A(f)$, such that $f$ is quasimeromorphic on $\overline{G(m, k)}$, we have $\overline{G(m, k)} \cap \overline{G\left(m, k^{\prime}\right)}=\varnothing$ whenever $k \neq k^{\prime}$, and for each $k \in \mathbb{N}$,

$$
\sup \left\{d_{\chi}(y, w(m, k)): y \in G(m, k)\right\} \rightarrow 0 \text { as } m \rightarrow \infty \text {. }
$$

Set $k_{1}=1$. For $m \in \mathbb{N}$, as $P\left(f ; e_{\sigma(m)}\right)$ is finite and the sets $G(m, k)$ have pairwise disjoint closures with respect to $k \in \mathbb{N}$, then there exists $k_{m+1} \in \mathbb{N}$ such that $\overline{G\left(m+1, k_{m+1}\right)} \cap P\left(f ; e_{\sigma(m)}\right)=\varnothing$. Now using Theorem 6.2.1 and a compactness argument, for each $m \in \mathbb{N}$, let $V(m)$ be an open set compactly contained in $f\left(G\left(m, k_{m}\right)\right) \backslash A(f)$ such that

$$
f(V(m)) \supset \overline{G\left(m+1, k_{m+1}\right)} .
$$


Note that as $f$ is continuous on $G(m, k)$ for all $m, k \in \mathbb{N}$, then equation (6.5.14) implies that

$$
\sup \left\{d_{\chi}\left(y, e_{\sigma(m)}\right): y \in V(m)\right\} \rightarrow 0 \text { as } m \rightarrow \infty
$$

Finally we inductively define the sets $F_{n}$ with $n \in \mathbb{N}$ as follows. Firstly, initialise $F_{0}=G(1,1)$ and then for each natural $n \geq 0$, define

$$
F_{n+1}= \begin{cases}V(m) & \text { if } F_{n}=G\left(m, k_{m}\right) \\ G\left(m+1, k_{m+1}\right) & \text { if } F_{n}=V(m) .\end{cases}
$$

Then considering equations (6.5.13) and (6.5.15), we can apply Lemma 6.4.4 with $\ell_{n}=1$ for all $n \in \mathbb{N}$ to our sets $F_{n}$ and $G_{n}=F_{n}$. This yields a point $x \in I_{g}(f)$ such that $\omega(x, f)=\left\{e_{n}: n \geq 0\right\}$, completing the proof of Theorem 6.5.3. 


\section{Bibliography}

[1] L.V Ahlfors: Zur Theorie der Überlagerungsflächen; Acta Math.; 65, 157-194 (1935).

[2] L. Ahlfors: On quasiconformal mappings; J. Anal. Math.; 3(1), 1-58 (1954).

[3] I.N. Baker: Wandering domains in the iteration of entire functions; Proc. London Math. Soc.; 49(3), 563-576 (1984).

[4] I.N. Baker, P. Domínguez and M.E. Herring: Dynamics of functions meromorphic outside a small set; Ergod. Th. E Dynam. Sys.; 21, 647-672 (2001).

[5] I.N. Baker, J. Kotus and Y. Lü: Iterates of meromorphic functions I.; Ergod. Th. Ef Dynam. Sys.; 11(2), 241-248 (1991).

[6] I.N. Baker, J. Kotus and Y. Lü: Iterates of meromorphic functions II. Examples of wandering domains; J. London Math. Soc.; 42(2), 267-278 (1990).

[7] I.N. Baker, J. Kotus and Y. Lü: Iterates of meromorphic functions III. Preperiodic domains; Ergod. Th. \&6 Dynam. Sys.; 11(4), 603-618 (1991).

[8] W. Bergweiler: Iteration of meromorphic functions; Bull. Amer. Math. Soc.; 29(2), 151-188 (1993).

[9] W. Bergweiler: An Introduction to Complex Dynamics; Universidade de Coimbra, Coimbra (1995). 
[10] W. Bergweiler: Fixed points of composite entire and quasiregular maps; Ann. Acad. Sci. Fenn. Math.; 31, 523-540 (2006).

[11] W. Bergweiler: Iteration of quasiregular mappings; Comput. Methods Funct. Theory; 10, 455-481 (2010).

[12] W. Bergweiler: Fatou-Julia theory for non-uniformly quasiregular maps; Ergod. Th. E Dynam. Sys.; 33(1), 1-23 (2013).

[13] W. Bergweiler, D. Drasin and A.N. Fletcher: The fast escaping set of a quasiregular mapping; Anal. Math. Phys.; 4, 83-98 (2014).

[14] W. Bergweiler, A.N. Fletcher, J. Langley and V. Mayer: The escaping set of a quasiregular mapping; Proc. Amer. Math. Soc.; 137, 641-651 (2009).

[15] W. Bergweiler, A.N. Fletcher and D.A. Nicks: The Julia set and the fast escaping set of a quasiregular mapping; Comput. Methods Funct. Theory; 14, 209-218 (2014).

[16] W. Bergweiler and A. Hinkkanen: On semiconjugation of entire functions; Math. Proc. Cambridge Philos. Soc.; 126, 565-574 (1999).

[17] W. Bergweiler and D.A. Nicks: Foundations for an iteration theory of entire quasiregular maps; Israel J. Math.; 201(1), 147-184 (2014).

[18] D.E. Blair: Inversion theory and conformal mapping (Student Mathematical Library, 9); American Mathematical Society, Rhode Island (2000).

[19] B. Bojarski and T. Iwaniec: Analytical foundations of the theory of quasiconformal mappings in $\mathbb{R}^{n}$; Ann. Acad. Sci. Fenn. Ser. A I Math.; 8, 257-324 (1983).

[20] A. Bolsch: Repulsive periodic points of meromorphic functions; Complex Var. Elliptic Equ.; 31(1), 75-79 (1996). 
[21] A. Bolsch: Iteration of meromorphic functions with countably many singularities; Dissertation, Technische Universität Berlin (1997).

[22] A. Bolsch: Periodic Fatou components of meromorphic functions; Bull. Lond. Math. Soc.; 31(5), 543-555 (1999).

[23] E.D. Callender Jr: Hölder continuity of $n$-dimensional quasiconformal mappings; Pacific J. Math.; 10(2), 499-515 (1960).

[24] P. Domínguez: Dynamics of transcendental meromorphic functions; Ann. Acad. Sci. Fenn. Math.; 23, 225-250 (1998).

[25] P. Domínguez, M.A. Montes de Oca and G. Sienra: Dynamics of meromorphic functions outside a countable set of essential singularities; preprint, 2017, available at https://arxiv.org/abs/1705.03960

[26] D. Drasin: The inverse problem of the Nevanlinna theory; Acta Math; 138, 83-151 (1977).

[27] D. Drasin and P. Pankka: Sharpness of Rickman's Picard theorem in all dimensions; Acta Math.; 214(2), 209-306 (2015).

[28] A.E. Eremenko: On the iteration of entire functions; Dynamical Systems and Ergodic Theory (Warsaw 1986) (Banach Center Publications, 23); PWN, Warsaw, 1989, pp. 339-345.

[29] A.E. Eremenko and M.Ju. Ljubich: Examples of entire functions with pathological dynamics; J. London Math. Soc.; 36(2), 458-468 (1987).

[30] V. Evdoridou, D. Martí-Pete, D. Sixsmith: Spiders' webs in the punctured plane; Ann. Acad. Sci. Fenn. Math.; 45, 511-531 (2020).

[31] L. Fang: On the iteration of holomorphic self-maps of $\mathbb{C}^{*}$; Acta Math. Sinica (N.S.); 14(1), 139-144 (1998). 
[32] P. Fatou: Sur les équations fonctionnelles; Bull. Soc. Math. France; 47, 161-271 (1919).

[33] P. Fatou: Sur l'itération des fonctions transcendantes entières; Acta Math.; 47, 337-360 (1926).

[34] A.N. Fletcher and D.A. Nicks: Quasiregular dynamics on the $n$-sphere; Ergod. Th. E Dynam. Sys.; 31, 23-31 (2011).

[35] A.N. Fletcher and D.A. Nicks: Iteration of quasiregular tangent functions in three dimensions; Conform. Geom. Dyn.; 16, 1-21 (2012).

[36] F.W. Gehring: Quasiconformal mappings in space; Bull. Amer. Math. Soc.; 69, 146-164 (1963).

[37] F.W. Gehring and J. Väisälä: The coefficients of quasiconformality of domains in space; Acta Math.; 114, 1-70 (1965).

[38] S. Granlund, P. Lindqvis and O. Martio: Conformally invariant variational integrals; Trans. Amer. Math. Soc.; 277(1), 43-73 (1983).

[39] W.K. Hayman: Meromorphic functions; Clarendon Press, Oxford (1964).

[40] J. Heinonen, T. Kilpeläinen and O. Martio: Nonlinear Potential Theory of Degenerate Elliptic Equations (Dover Edition); Dover publications, New York (2006).

[41] J. Heinonen and P. Koskela: Weighted Sobolev and Poincaré inequalities and quasiregular mappings of polynomial type; Math. Scand.; 77, 251-271 (1995).

[42] M.E. Herring: An extension of the Julia-Fatou theory of iteration; PhD Thesis, Imperial College London (1994).

[43] A. Hinkkanen, G. Martin and V. Meyer: Local dynamics of uniformly quasiregular mappings; Math. Scand.; 95, 80-100 (2004). 
[44] I. Holopainen: Nonlinear potential theory and quasiregular mappings on Riemannian manifolds; Ann. Acad. Sci. Fenn. Ser. A I Math. Diss.; 74, 1-45 (1990).

[45] W. Hurecwitz and H. Wallman: Dimension Theory; Princeton University Press, New Jersey (1948).

[46] T. Iwaniec: Some aspects of partial differential equations and quasiregular mappings; Proc. Internat. Congr. Math. vol. 2, Warsaw, pp. 1193-1208 (1983); PWN, Warsaw (1984).

[47] T. Iwaniec, G. Martin: Quasiregular mappings in even dimensions; Acta Math.; 170, 29-81 (1993).

[48] T. Iwaniec and G. Martin: Geometric Function Theory and Non-linear Analysis (Oxford Mathematical Monographs); Oxford University Press, Oxford (2001).

[49] T. Iwaniec, L. Migliaccio, L. Nania and C. Sbordone: Integrability and removability results for quasiregular mappings in high dimensions; Math. Scand.; 75, 263-279 (1994).

[50] P. Järvi: On the beaviour of quasiregular mappings in the neighbourhood of an isolated essential singularity; Ann. Acad. Sci. Fenn. Ser. A I Math.; 15, 341-353 (1990).

[51] P. Järvi: On the zeros and growth of quasiregular mappings; J. Anal. Math.; 82, 347-362 (2000).

[52] G. Julia: Sur l'itérations des fonctions rationnelles; J. Math. Pures Appl. Ser. $8 ; 1,47-246(1918)$. 
[53] S. Karlin and J. McGregor: Embedding iterates of analytic functions with two fixed points into continuous groups; Trans. Amer. Math. Soc.; 132, 137-145 (1968).

[54] J. Liouville: Théorème sur l'équation $d x^{2}+d y^{2}+d z^{2}=\lambda\left(d \alpha^{2}+d \beta^{2}+d \gamma^{2}\right)$; J. Math. Pures Appl.; 1(15), 103 (1850).

[55] J.E. Littlewood and A.C. Offord: On the distribution of zeros and $a$-values of a random integral function (II); Ann. Math. $2^{\text {nd }}$ Series; 49(4), 885-952 (1948).

[56] D. Martí-Pete: The escaping set of transcendental self-maps of the punctured plane; Ergod. Th. ES Dynam. Sys.; 38, 739-760 (2018).

[57] O. Martio: Partial differential equations and quasiregular mappings; Lecture Notes in Mathematics, vol. 1508, pp. 65-79; Springer-Verlag (1992).

[58] O. Martio, S. Rickman and J. Väisälä: Definitions for quasiregular mappings; Ann. Acad. Sci. Fenn. Ser. A I Math.; 448, 1-40 (1969).

[59] O. Martio, S. Rickman and J. Väisälä: Distortion and singularities of quasiregular mappings; Ann. Acad. Sci. Fenn. Ser. A I Math.; 465, 1-13 (1970).

[60] O. Martio, S. Rickman and J. Väisälä: Topological and metric properties of quasiregular mappings; Ann. Acad. Sci. Fenn. Ser. A I Math.; 488, 1-31 (1971).

[61] O. Martio, U. Srebro: Automorphic quasimeromorphic mappings in $\mathbb{R}^{n} ;$ Acta Math.; 135, 221-247 (1975).

[62] O. Martio and U. Srebro: Periodic quasimeromorphic mappings in $\mathbb{R}^{n} ; J$. Anal. Math.; 28(1), 20-40 (1975).

[63] J. Milnor: Dynamics in One Complex Variable, $3^{\text {rd }}$ Edition (Annals of Mathematics Studies, no.160); Princeton University Press, Oxford (2006). 
[64] R. Miniowitz: Normal families of quasimeromorphic mappings; Proc. Amer. Math. Soc.; 84(1), 35-43 (1982).

[65] R. Nevanlinna: Zur Theorie der meromorphen Funktionen; Acta Math.; 46, 1-99, (1925).

[66] D.A. Nicks: Slow escaping points of quasiregular mappings; Math. Z.; 284, 1053-1071 (2016).

[67] D.A. Nicks and D.J. Sixsmith: The size and topology of quasi-Fatou components of quasiregular maps; Proc. Amer. Math. Soc.; 145(2), 749-763 (2016).

[68] D.A. Nicks and D.J. Sixsmith: Hollow quasi-Fatou components of quasiregular maps; Math. Proc. Camb. Phil. Soc.; 162, 561-574 (2017).

[69] D.A. Nicks and D.J. Sixsmith: Periodic domains of quasiregular maps; Ergod. Th. \& Dynam. Sys.; 38(6), 2321-2344 (2018).

[70] D.A. Nicks and D.J. Sixsmith: The dynamics of quasiregular maps of punctured space; Indiana Univ. Math. J; 68(1), 323-352 (2019).

[71] D.A. Nicks and D.J. Sixsmith: The bungee set in quasiregular dynamics; Bull. London Math. Soc.; 51(1), 120-128 (2019).

[72] Y. Okuyama and P. Pankka: Accumulation of periodic points for local uniformly quasiregular mappings; RIMS Kôkyûroku Bessatsu; B43, 121-139 (2013).

[73] J.W. Osborne: Connectedness properties of the set where the iterates of an entire function are bounded; Math. Proc. Cambridge Philos. Soc.; 155(3), 391-410 (2013).

[74] J.W. Osborne and D.J. Sixsmith: On the set where the iterates of an entire function are neither escaping nor bounded; Ann. Acad. Sci. Fenn. Math; 41(2), 561-578 (2016). 
[75] M.I. Pesonen: Simplified proofs of some basic theorems for quasiregular mappings; Ann. Acad. Sci. Fenn. Ser. A I Math.; 8, 247-250 (1983).

[76] T. Radó and P.V. Reichelderfer: Continuous Transformations in Analysis (Grundlehren der math. Wissenschaften, 75); Springer, Berlin (1955).

[77] L. Rempe: The escaping set of the exponential; Ergod. Th. \& Dynam. Sys.; 30(2), 595-599 (2010).

[78] Y. Reshetnyak: Space mappings with bounded distortion; Sibirsk. Mat. Zh.; 8 (1967), 629-659.

[79] Y. Reshetnyak: On the condition of the boundedness of index for mappings with bounded distortion; Sibirsk. Mat. Zh.; 9, 368-374 (1968).

[80] Y. Reshetnyak: The concept of capacity in the theory of functions with bounded distortion; Sibirsk. Mat. Zh.; 10, 1300-1310 (1969).

[81] Y. Reshetnyak: The local structure of mappings with bounded distortion; Sibirsk. Mat. Zh.; 10, 1311-1313 (1969).

[82] Y. Reshetnyak: On the branch set of mappings with bounded distortion; Sibirsk. Mat. Zh.; 11, 1333-1339 (1970).

[83] S. Rickman: On the number of omitted values of entire quasiregular mappings; J. Anal. Math.; 37, 100-117 (1980).

[84] S. Rickman: Value distribution of quasiregular mappings; Proc. Value Distribution Theory, Joensuu 1981. Lecture Notes in Mathematics, vol. 981, pp. 220-245; Springer, Berlin (1983).

[85] S. Rickman: The analogue of Picard's theorem for quasiregular mappings in dimension three; Acta Math.; 154, 195-242 (1985). 
[86] S. Rickman: Defect relation and its realization for quasiregular mappings; Ann. Acad. Sci. Fenn. Ser. A I Math.; 20, 207-243 (1992).

[87] S. Rickman: Quasiregular mappings (Ergebnisse der Mathematik und ihrer Grenzgebiete, 26); Springer, Berlin (1993).

[88] P.J. Rippon and G.M. Stallard: On sets where iterates of a meromorphic function zip towards infinity; Bull. London Math. Soc.; 32, 528-536 (2000).

[89] P.J. Rippon and G.M. Stallard: Escaping points of meromorphic functions with a finite number of poles; J. Anal. Math.; 96, 225-245 (2005).

[90] P.J. Rippon and G.M. Stallard: Dimensions of Julia sets of meromorphic functions; J. London Math. Soc.; 71(2), 669-683 (2005).

[91] P.J. Rippon, G.M. Stallard: On questions of Fatou and Eremenko; Proc. Amer. Math. Soc.; 133, 1119-1126 (2005).

[92] P.J. Rippon and G.M. Stallard: Escaping points of entire functions of small growth; Math. Z.; 261(3), 557-570 (2009).

[93] P.J. Rippon and G.M. Stallard: Slow escaping points of meromorphic functions; Trans. Am. Math. Soc.; 363(8), 4171-4201 (2011).

[94] G. Rottenfusser, J. Rückert, L. Rempe and D. Schleicher: Dynamic rays of bounded-type entire functions; Ann. Math.; 173(1), 77-125 (2011).

[95] H. Siebert: Fixpuntke und normale Familien quasiregulärer Abbildungen; Dissertation, University of Kiel (2004).

[96] D.J. Sixsmith: Entire functions for which the escaping set is a spider's web; Math. Proc. Cambridge Philos. Soc.; 151(3), 551-571 (2011). 
[97] D.J. Sixsmith: Maximally and non-maximally fast escaping points of transcendental entire functions; Math. Proc. Cambridge Philos. Soc.; 158(2), 365-383 (2015).

[98] D.J. Sixsmith: Dynamical sets whose union with infinity is connected; Ergod. Th. \& Dynam. Sys.; 40(3), 789-798 (2020).

[99] D. Sun and L. Yang: Quasirational dynamic system; Chinese Science Bull.; 45, 1277-1279 (2000).

[100] D. Sun and L. Yang: Iteration of quasi-rational mapping; Prog. Natur. Sci. (English Ed.); 11(1), 16-25 (2001).

[101] J. Väisälä: On quasiconformal mappings in space; Ann. Acad. Sci. Fenn. Ser. A $I ; \mathbf{2 9 8 , 1 - 3 6 ~ ( 1 9 6 1 ) . ~}$

[102] J. Väisälä: Discrete open mappings on manifolds; Ann. Acad. Sci. Fenn. Ser. A I; 392, 1-10 (1966).

[103] M. Vuorinen: Some inequalities for the moduli of curve families; Michigan Math. J.; 30, 369-380 (1983).

[104] M. Vuorinen: Conformal invariants and quasiregular mappings; J. Anal. Math.; 45, 69-115 (1985).

[105] M. Vuorinen: Conformal Geometry and Quasiregular Mappings; Lecture Notes in Mathematics, vol. 1319; Springer, Berlin (1988).

[106] H. Wallin: Metrical characterization of conformal capacity zero; J. Math. Anal. Appl.; 58(2), 298-311 (1977).

[107] L. Warren: On the iteration of quasimeromorphic mappings; Math. Proc. Cambridge Philos. Soc.; 168(1), 1-11 (2020). 
[108] L. Warren: On slow escaping and non-escaping points of quasimeromorphic mappings; Ergod. Th. \& Dynam. Sys.; [Online] Cambridge University Press; 1-27 (2020), available at doi:10.1017/etds.2019.110

[109] L. Warren: Constructing a quasiregular analogue of $z \exp (z)$ in dimension 3; submitted, 2019, available at https://arxiv.org/abs/1907.04720

[110] J. Waterman: Slow escape in tracts; Proc. Amer. Math. Soc.; 147, 3087-3101 (2019).

[111] V.A. Zorich: A theorem of M. A. Lavrent'ev on quasiconformal maps; Math. Sb.; 74(3), 417-433 (1967). 